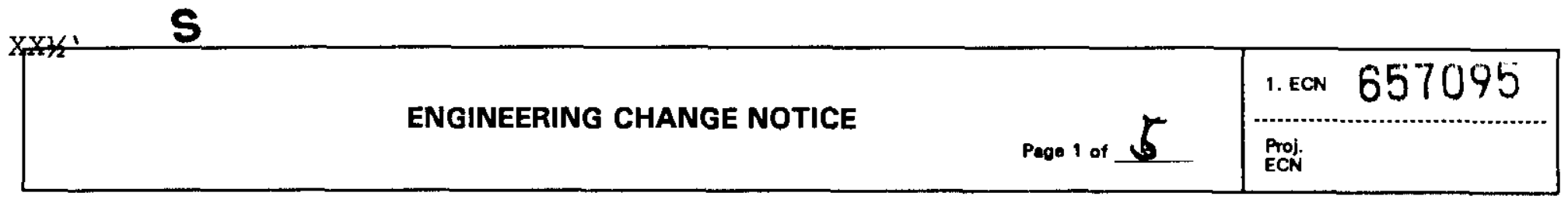

\begin{tabular}{|c|c|c|c|c|}
\hline \multicolumn{2}{|l|}{$\begin{array}{l}\text { Direct Revision } \\
\text { Change ECN } \\
\text { Temporary } \\
\text { Standby } \\
\text { Supersedure } \\
\text { Cancel/Void }\end{array}$} & \multirow{3}{*}{$\begin{array}{l}\text { 3. Originator's Name, Organization, } \\
\text { MSIN, and Telephone No. } \\
\text { H.R. Risenmay, Stabilization, } \\
\text { T5-55, } 373-3503 \\
\text { 6. Project Title/No. Work Order No. } \\
\text { Thermal. Stabilization } \\
\text { 9. Document Numbers Changed by this ECN } \\
\text { (includes sheet no. and rev.) } \\
\text { HNE-4989, Rev. O }\end{array}$} & & \\
\hline \multirow{2}{*}{$\begin{array}{l}\text { Direct Revision } \\
\text { Change ECN } \\
\text { Temporary } \\
\text { Standby } \\
\text { Supersedure } \\
\text { Cancel/Void }\end{array}$} & \multirow{2}{*}{$\begin{array}{r}{[X]} \\
{[]} \\
{[]} \\
{[]} \\
{[]} \\
{[]}\end{array}$} & & & \\
\hline & & & $\begin{array}{l}\text { 4. USO Required? } \\
\text { [X] Yes [] No }\end{array}$ & $\begin{array}{l}\text { 5. Date } \\
\text { April 19, } 2000\end{array}$ \\
\hline
\end{tabular}

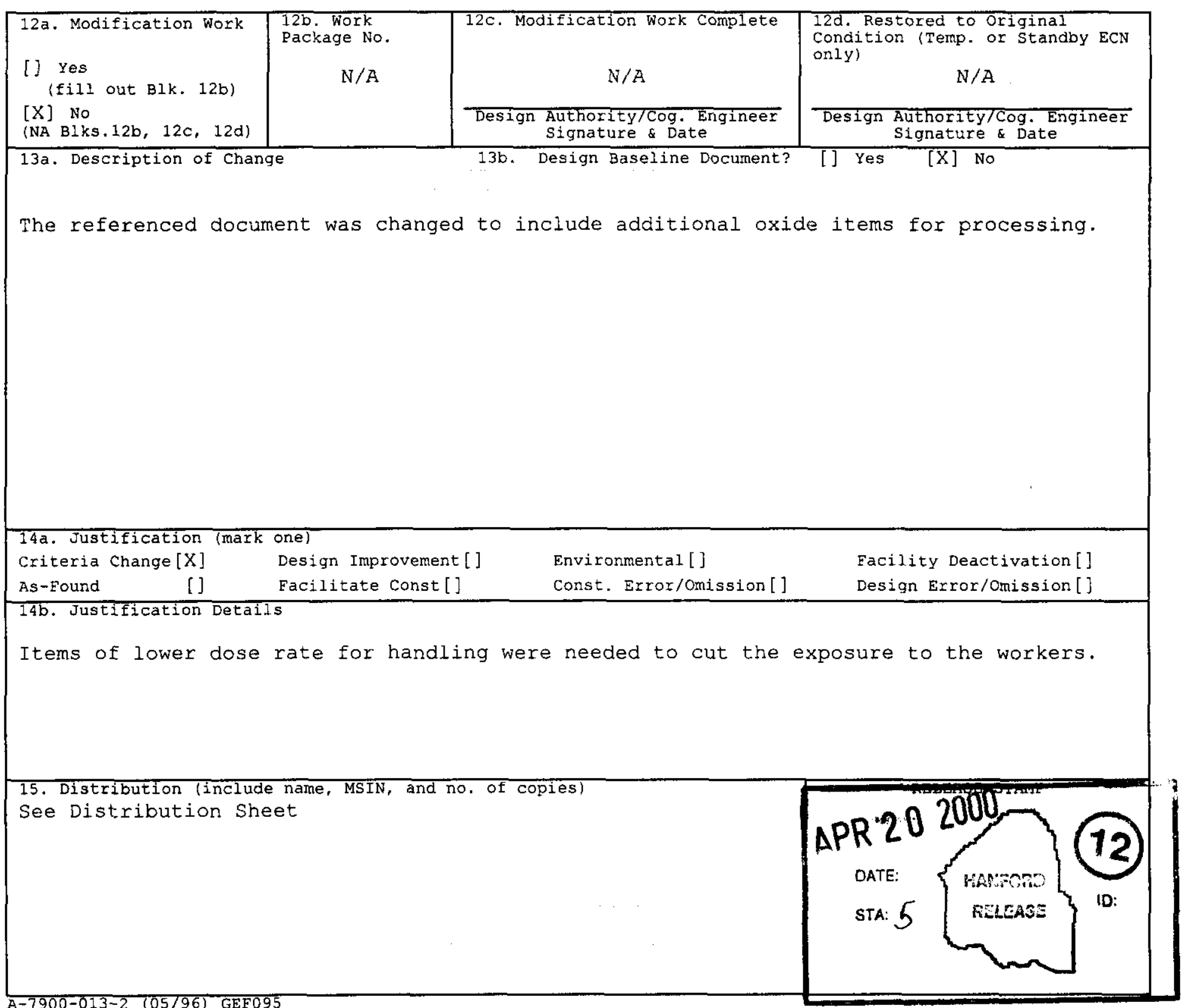




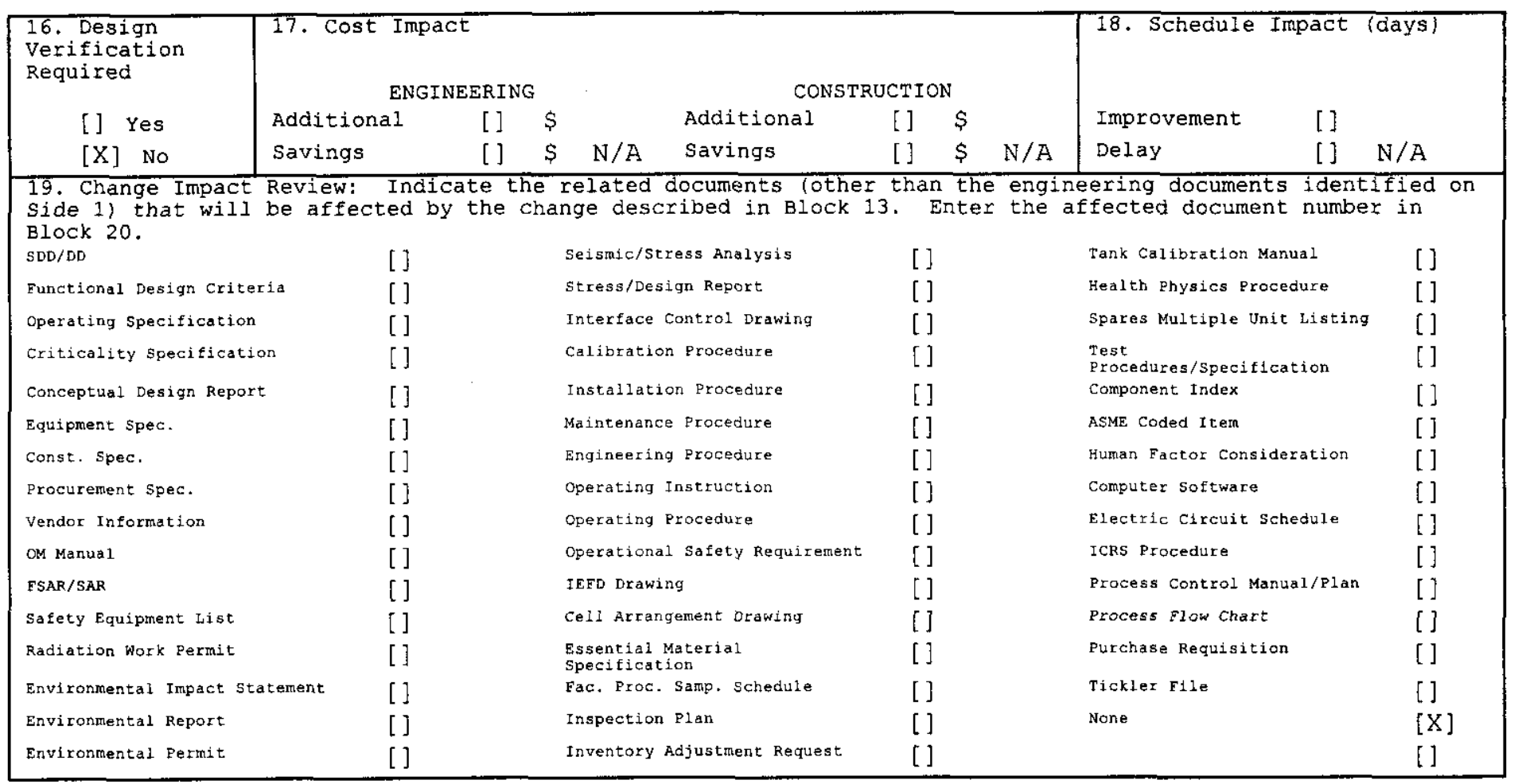

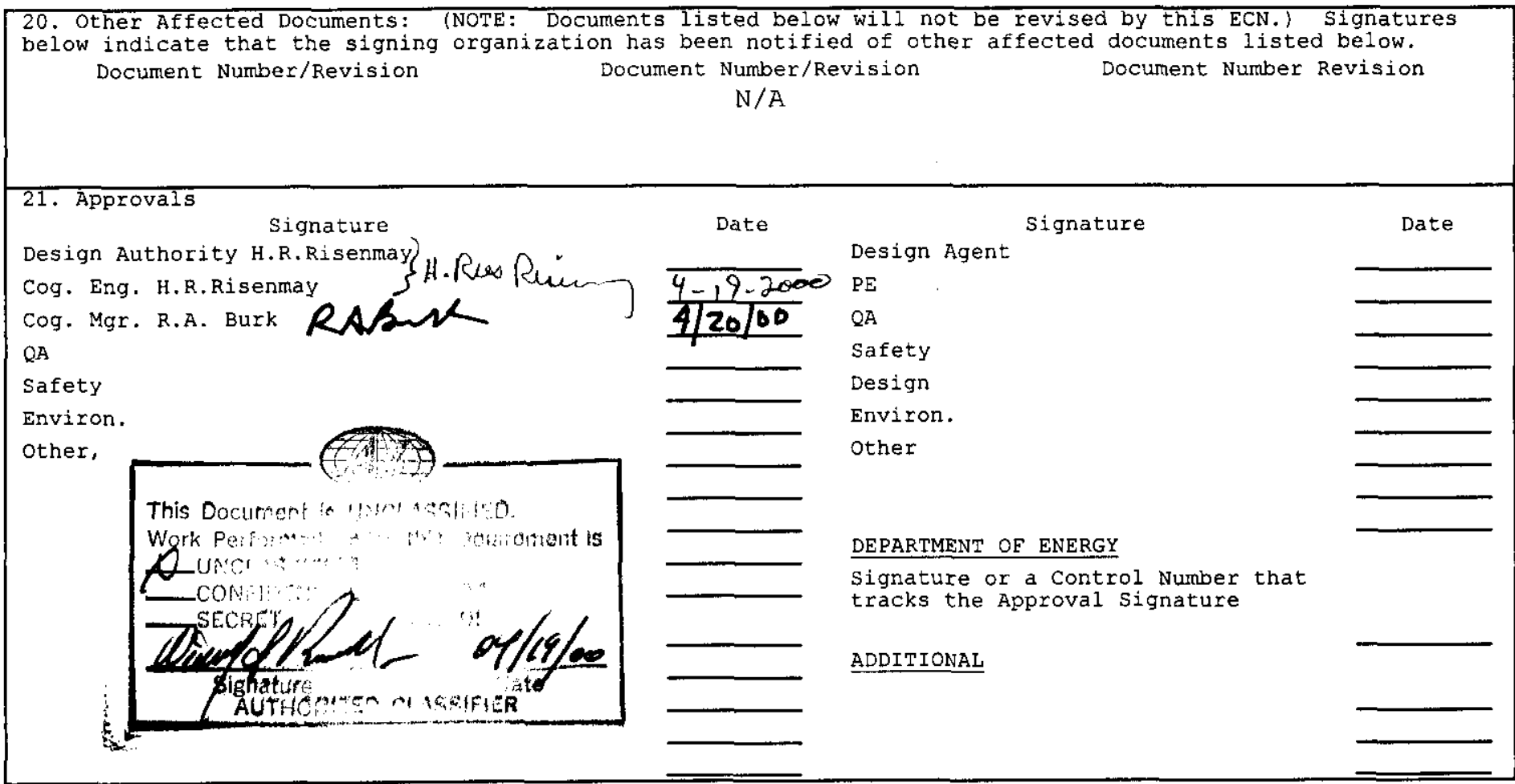




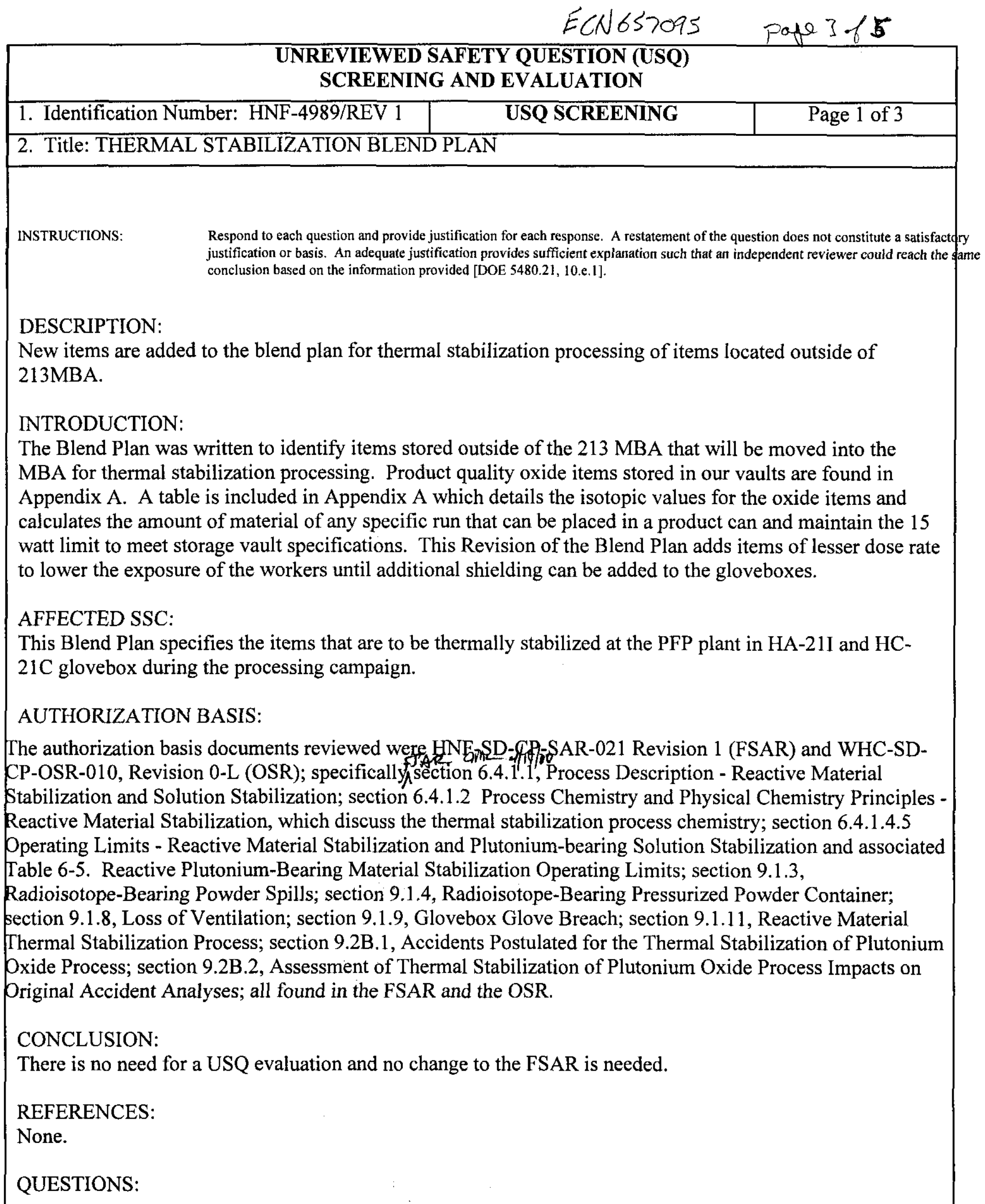

1.Does the proposed change or occurrence represent a change to the facility or procedures as described in the 


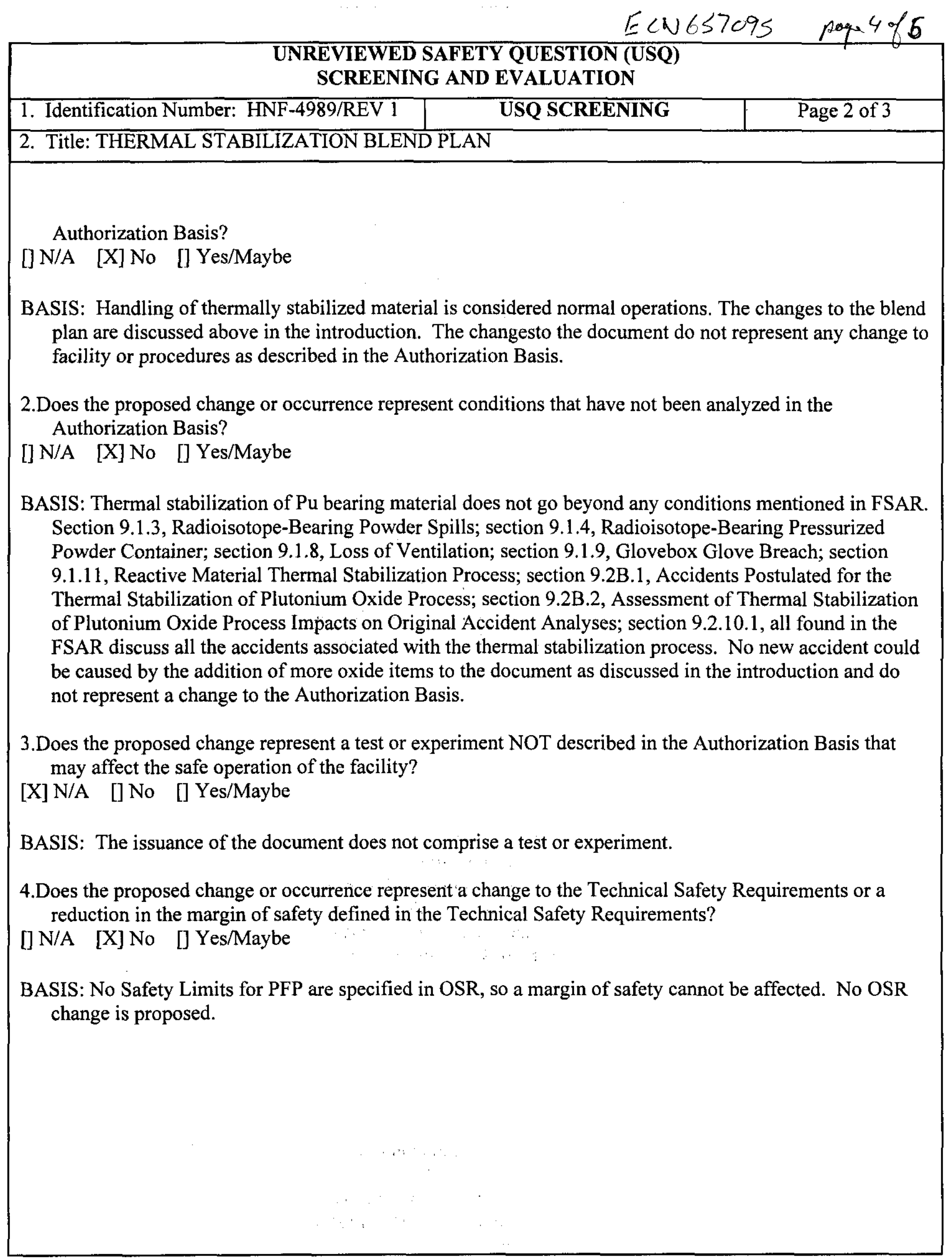




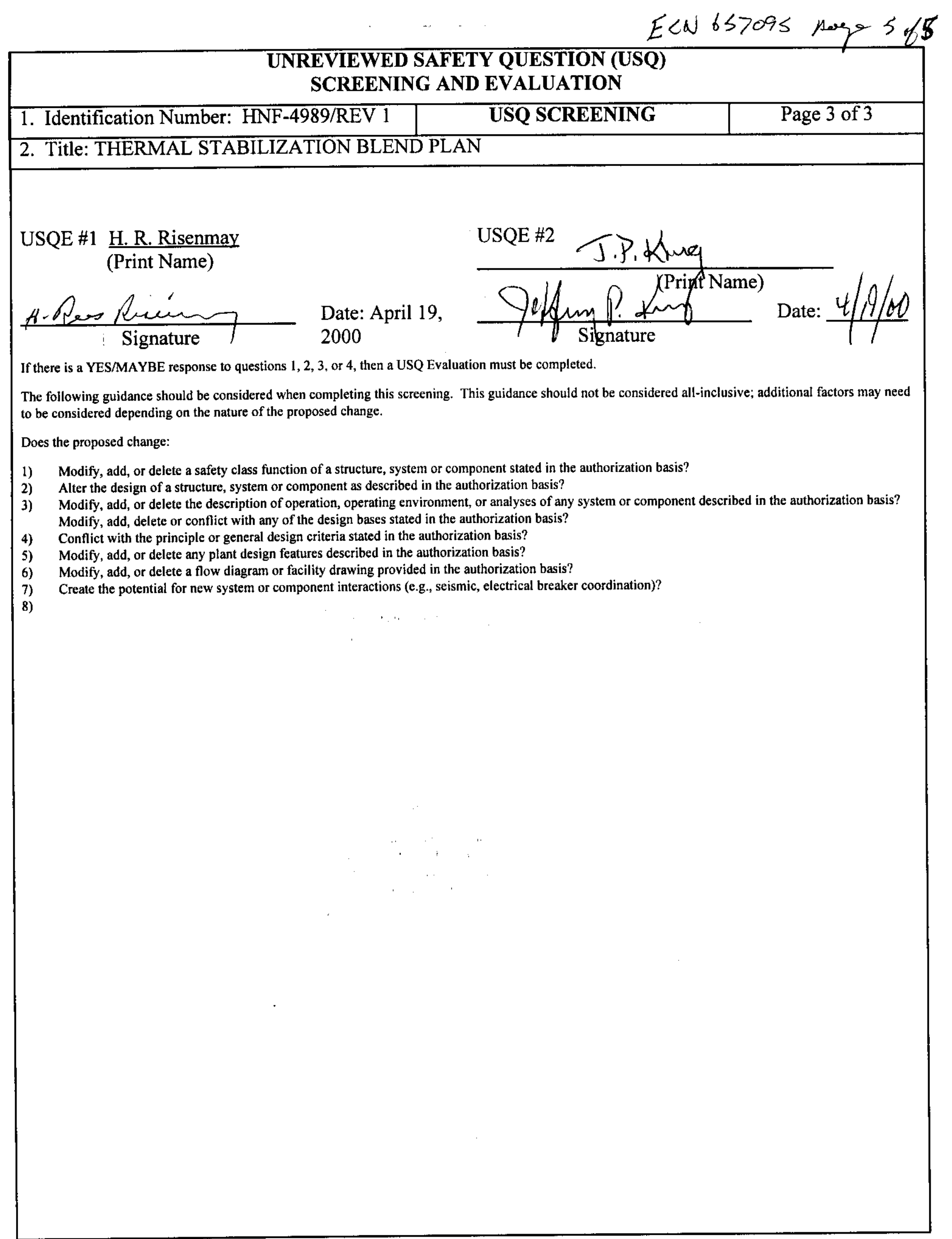


HNF-4989

Revision 1

\section{Thermal Stabilization Blend Plan}

Prepared for the U.S. Department of Energy Assistant Secretary for Environmental Management

Project Hanford Management Contractor for the

U.S. Department of Energy under Contract DE-AC06-96RL13200

Fluor Hanford

P.O. Box 1000

Richland, Washington

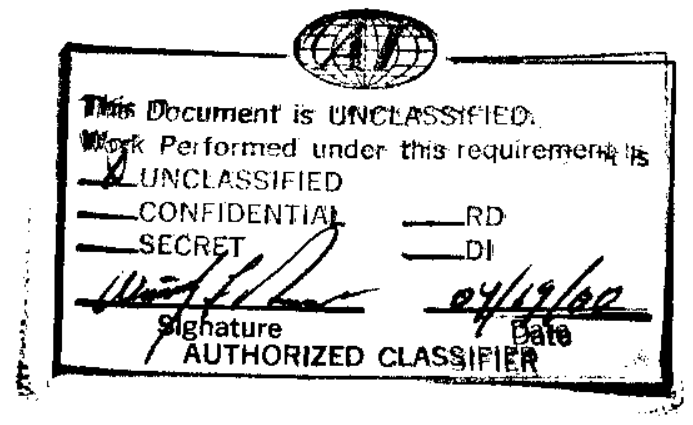


HNF-4989

Revision 1

ECN 657095

\section{Thermal Stabilization Blend Plan}

H. R. Risenmay

Fluor Hanford

Date Published

April 2000

Prepared for the U.S. Department of Energy

Assistant Secretary for Environmental Management

Project Hanford Management Contractor for the

U.S. Department of Energy under Contract DE-AC06-96RL13200

Fivor Hanford

P.O. Box 1000

Richland, Washington
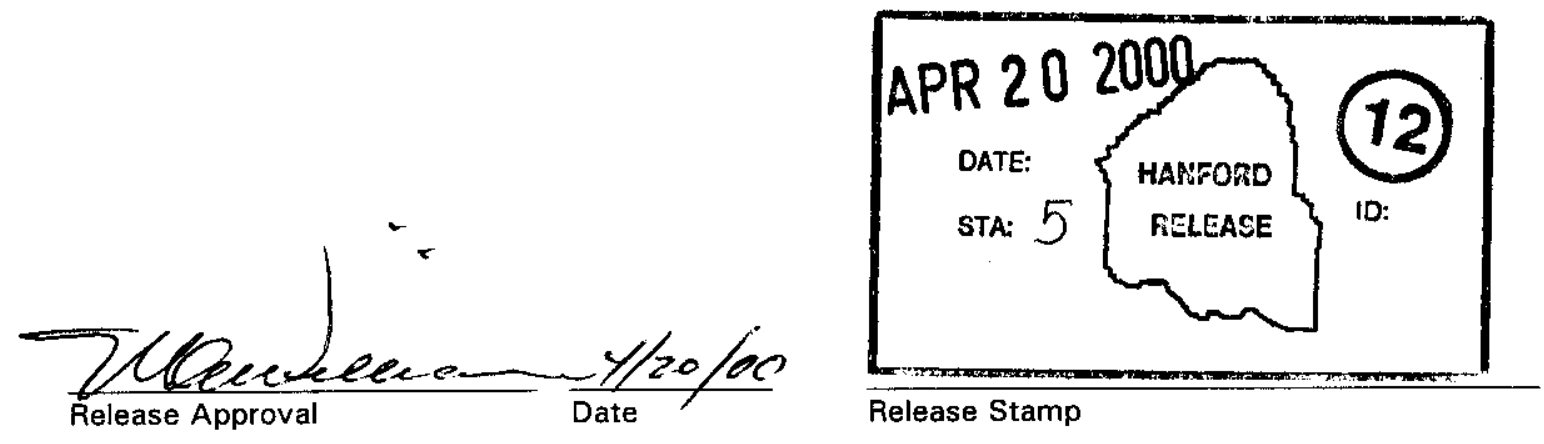
HNF-4989

Revision 1

LEGAL DISCLAIMER

This report was prepared as an account of work sponsored by an agency of the United States Government. Neither the United States Government nor any agency thereof, nor any of their employees, nor any of their contractors, subcontractors or their employees, makes any warranty, express or implied, or assumes any legal liability or responsibility for the accuracy, completeness, or any third party's use or the results of such use of any information, apparatus, product, or process disclosed, or represents that its use would not infringe privately owned rights. Reference herein to any specific commercial product, process, or service by trade name, trademark, manufacturer, or otherwise, does not necessarily constitute or imply its endorsement, recommendation, or favoring by the United States Government or any agency thereof or its contractors or subcontractors. The views and opinions of authors expressed herein do not necessarily state or reflect those of the United States Government or any agency thereof.

This report has been reproduced from the best available copy.

Printed in the United States of America

Total Pages: $\quad 54$ 
(2) Title

THERMAL STABILIZATION BLEND PLAN

CHANGE CONTROL RECORD

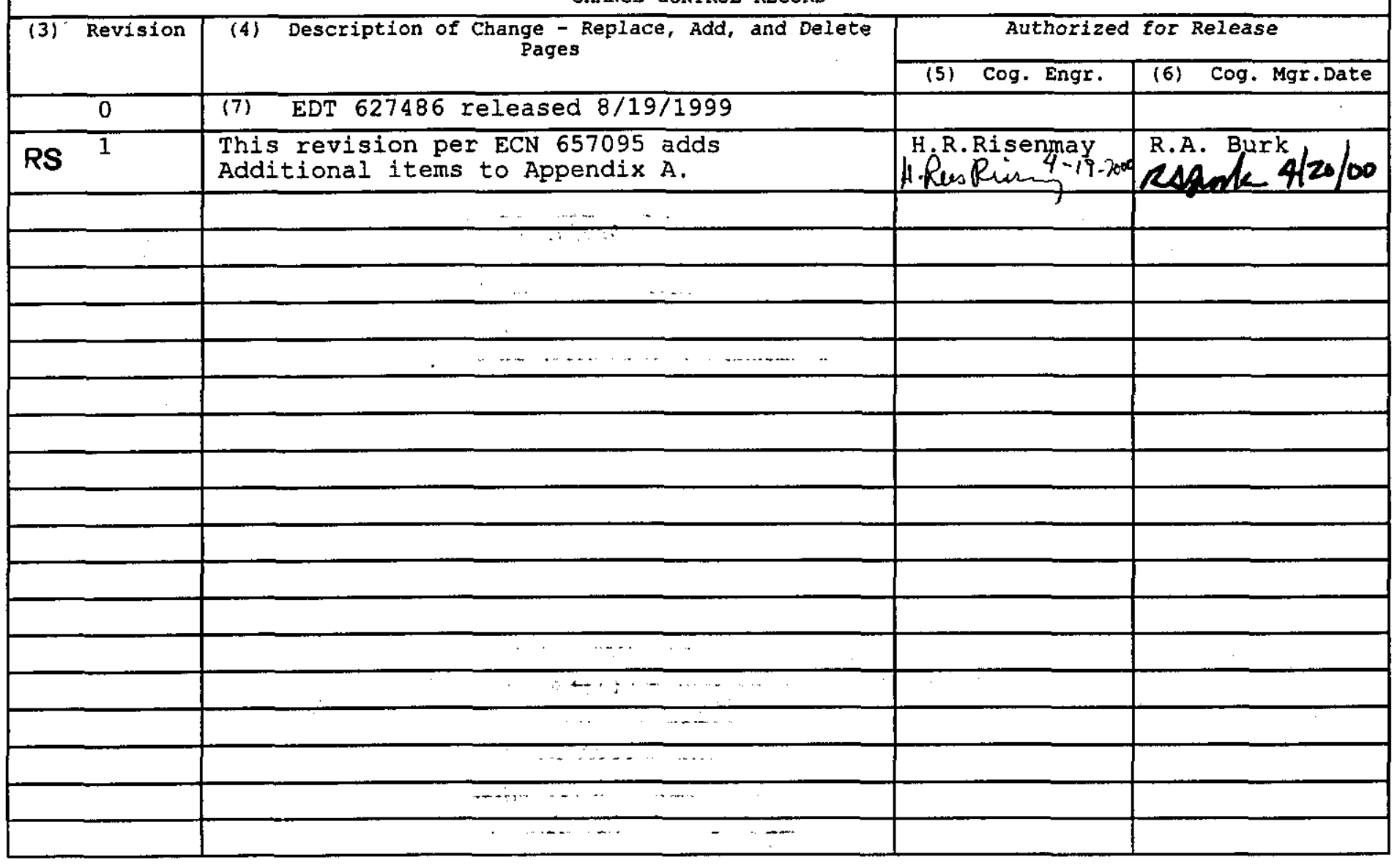




\begin{tabular}{||l|l|l|}
\hline PFP THERMAL & \multicolumn{1}{|c|}{ BLEND PLAN } & $\begin{array}{l}\text { HNF-4989, REV. 1 } \\
\text { PAGE 1 OF 51 }\end{array}$ \\
\hline
\end{tabular}

TABLE OF CONTENTS

I. INTRODUCTION

PAGE

II. MATERIAL TO BE PROCESSED

2

III. PROCESS ORDER

IV. REFERENCES 


\begin{tabular}{|l|l|l|}
\hline $\begin{array}{l}\text { PFP THERMAL } \\
\text { STABILIZATION }\end{array}$ & BLEND PLAN & $\begin{array}{l}\text { HNF-4989, REV. 1 } \\
\text { PAGE 2 OF 51 }\end{array}$ \\
\hline
\end{tabular}

\section{INTRODUCTION}

This Blend Plan documents the feed material items for the themation are tocated outside the 213 MBA. Items within the $213 \mathrm{MBBA}$ will be found in the classified Blend Plan, NAF-3425. This blend plan lists plutmoxide-stored in 2736-Z vaults, the 2736-ZB 638 cage, the $192 \mathrm{C}$ vault, and the 225 vault that will be processed through the thermal stabilization furnaces.

The purpose of thermal stabilization is to heat the material to 1000 degrees Celsius to drive off all water alatile materials and leave the plutonium and/or uranium as oxides. The stabilized material will be sampled to determine the Loss On Ignition (LOI) or percent water. The stabilized material must meet water content or LOI of less than $0.5 \%$ to be acceptable for storage under DOE-STD-3013-994 specifications. Out of specification material will be recycled through the furnaces until the water or LOI limits are met.

\section{MATERIAL TO BE PROCESSED}

Feed material and items were selected using the criteria in the Process Control Plan (Reference). The list in the appendix reflect the application of that criteria to the data available for ach item. A elassified database containing lemental weights and locations is separately available to assist operations andengineering in detailed selections.

\section{A. OXIDES}

Theis bulk of this material is currently stored in the 2736-Z Vaults. Additional items are found in the 638 cage, 225 vault, and the $192 \mathrm{C}$ vault. Radiographs of some of the items indicates that some of the material is in a lead cup inside a slip lid can, a plastic bag, and two clean overpack cans. Others are in any combination of 2 to 4 overpacked cans. The material will be processed to meet DOE-STD-3013-994 specifications.

The batch limits imposed by the OSD on oxides are 2725 grams net weight per batch containing 2400 grams of $\mathrm{Pu}$. The EIS and FSAR analysis for thermal stabilization have been revised in HNF-SD-CP SAR 021, Rev. 1, and have been approved by DOE. The new timits are based on the criticality limits for both the individual boat/batch and the glovebox. -The total Pu element weight allowed in the HC-21C glovebox by the criticality specification is 5000 grams. The total Pu element weight allowed in each individual boat/batch by the criticality specification is 2500 grams. This does not give any room for glovebox holdup. The typical glovebox holdup is about 50 grams $\mathrm{Pu}$. The limit for each boat has been set at 2400 grams $\mathrm{Pu}$ per batch by the OSD which will allow 200 grams $\mathrm{Pu}$ for $\mathrm{HC}-21 \mathrm{C}$ glovebox holdup when two boats are being processed. Glovebox HA-21I has a glovebox limit of 7500 grams Pu which gives 300 grams available for holdup when three boats are in process. To simplify calculations needed during processing, the material is assumed to be $88 \% \mathrm{Pu}$ (typical of product quality oxide) which resulted in the net weight batch size of 2725 grams specified in the OSD. The items selected for processing will meet these limits by splitting and combining items as needed during the furnace charge 


\begin{tabular}{|l|l|l|}
\hline $\begin{array}{l}\text { PFP THERMAL } \\
\text { STABILIZATION }\end{array}$ & BLEND PLAN & $\begin{array}{l}\text { HNF-4989, REV. 1 } \\
\text { PAGE 3 OF 51 }\end{array}$ \\
\hline
\end{tabular}

preparation process.

Some of the items in Appendix $\mathrm{AB}$ can be loaded into the PUREX slip lid can to exceed the vault storage specification of 15 watts/item for thermal loading. Appendix $\underline{A} B$ includes a table to be used with the Process Traveler to specify the maximum material that can be combined into one item when processing the oxide items. However, all the information on isotopic analysis was not available for all the items included in Appendix $\underline{A} B$. The isotopic analysis will need to be determined as items are prepared for transfer to $234-5 \mathrm{Z}$ for thermal stabilization. As the missing data in the table at the front of Appendix $\mathrm{AB}$ becomes available the data the-will be recalculated and the tablewill be reprinted as a separate spreadsheet that and can then be used for determining allowable loading of the stabilized material in the product cans. The table in Appendix $\underline{A} B$ will be used to determine which items need to have isotopic analysis performed before transfer to $234-5 \mathrm{Z}$ for processing.

Ordinarily, material will be processed as batches when those batches can be identified. Much of the oxide material was produced as batches and blended in a $\mathrm{V}$ blender batch and loaded into product cans. The product cans are identified with the same batch number with the difference in ID number being the can number for the item.

When it is not possible to identify batches the following criteria will be used to determine what materials can be mixed together in the boat charge makeup or filling of the product cans:

The isotopic contents of the original items must be similar, i.e:

- The Am-241 range cannot be greater than $0.4 \%$

- The Pu-238 range cannot be greater than $0.11 \%$

- The Pu-240 range cannot be greater than $5 \%$

If only the isotopic analysis for $\mathrm{Pu} 240$ is available then that will be sufficient. The criteria above for Pu 240 will be used to determine what material can be mixed together. 


\begin{tabular}{|l|l|l|}
\hline $\begin{array}{l}\text { PFP THERMAL } \\
\text { STABILIZATION }\end{array}$ & BLEND PLAN & $\begin{array}{l}\text { HNF-4989, REV. 1 } \\
\text { PAGE 4 OF 51 }\end{array}$ \\
\hline
\end{tabular}

\section{PROCESS ORDER}

Items to be processed through thermal stabilization are found in appendix A. The exact order of processing will be specified in the approved Process Traveler Sheets referred to in the Operating Instructions (OI) and will be based on guidance provided in this document 3425. Operations and Process-the CogEngineering/STA will concur/determine items to process and approve each OI. The Process Travelers and the daily OI should take into consideration good ALARA practices to keep radiation dose and handling of items to a minimum. This would include processing of items from the same vault location at the same time when possible, but all material from one batch will be processed sequentially. This could entail accessing several vault locations to retrieve the necessary items for processing. Other criteria in the Process Control Plan will ats $\theta$ be athere to during the selection of items hay.

\section{REFERENCES}

Process Control Plan for FY 1999 Themal Stabilization, HNF-3393, Rev. 1; B\&W Hanford Company; Richland, Washington; December 2, 1998

Thermal Stabilization Glovebox HC-21C; OSD-Z-184-00006, Rev. D-63; B\&W Hanford Company; Richland, Washington; August 19, 1999.

Thermal Stabilization Process Flowsheet; PFD-Z-190-00004, Rev D-24; B\&W Hanford Company; | Richland, Washington; February 9, 1999.

Plutonium Finishing Plant Stabilization Final Environmental Impact Statement, DOE/EIS-0244-F; Department of Energy, Richland Operations Office, Richland, Washington; May, 1996.

PFP Final Safety Analysis Report, HNF-SD-CP-SAR-021, Rev. 1, B\&W Hanford Company, Richland, Washington, January 11, 1999. 


\begin{tabular}{|l|l|l||}
\hline $\begin{array}{l}\text { PFP THERMAL } \\
\text { STABILIZATION }\end{array}$ & BLEND PLAN & $\begin{array}{l}\text { HNF-4989, REV. 1 } \\
\text { PAGE 5 OF 51 }\end{array}$ \\
\hline
\end{tabular}

APPENDIX A

OXIDE ITEMS LOCATED IN 2736-Z VAULTS

In following tables:

CAT $\underline{5=\text { WG Plutonium conversion other scrap }}$

6= FG Plutonium conversion other scrap

$10=$ MBA 212 PMP scrap

$20=$ FG Pu rec miscellaneous scrap

$41=$ WG Analytical laboratories miscellaneous scrap

$42=$ FG Analytical laboratories miscellaneous scrap

$51=$ WG Ctl miscellaneous scrap

$\underline{52=\text { FG Ctl miscellaneous scrap }}$

$61=$ Stabilized oxide

$\underline{62=\text { Stabilized Scrap }}$

$\underline{63=\text { Stabilized PRF scrap }}$

$64=$ Stabilized $C$ line scrap

$67=$ FG BNW scrap pwd

$73=$ WG BNW miscellaneous scrap

$74=$ FG BNW miscellaneous scrap

$91=$ HEDL Pu oxide scrap

$102=$ WG oxide for recovery

111 = BNW items for storage

$112=$ HEDL oxide for storage

$114=$ Oxide fuels grade A line

$145=$ Miscellaneous fuels grade samples

$\underline{148=\text { Miscellaneous fuels grade oxide }}$

$150=$ Burned button oxide

$173=$ Oxide fuels grade

$175=$ Oxide fuels grade other

$451=303-\mathrm{c}$ scrap from BNW

$455=$ FG Pu oxide contaminated

$465=$ WG plutonium-aluminum oxide or scrap

$482=$ FG Plutonium oxide

$849=$ Radiation sources for recovery

$\underline{850=\text { Radiation sources }}$

COEI: $454=$ Dioxides product

$455=$ Other oxides product

$746=$ In other R\&D usage

$771=$ Samples and standards

$\mathrm{C} 00=$ Plutonium nonconforming compounds

$\mathrm{C} 01=$ Oxides (low fired, relatively pure) 


\begin{tabular}{|l|c|l|}
\hline \hline $\begin{array}{l}\text { PFP THERMAL } \\
\text { STABILIZATION }\end{array}$ & BLEND PLAN & $\begin{array}{l}\text { HNF-4989, REV. 1 } \\
\text { PAGE 6 OF 51 }\end{array}$ \\
\hline
\end{tabular}

$\mathrm{C02}=$ Oxides (high fired)
C30=Encapsulated plutonium compounds/UR carbides
G00= Nonconforming residues
G05 = Reduction residues/residues to be leached

The following item category codes are weapons grade (WG) material and no wattage limits are necessary. Items with the same category code can also be mixed together without problems regarding the isotopic values:

\begin{tabular}{llll}
102 & 28 & 36 & 41 \\
\hline 42 & 43 & 5 & 51 \\
\hline 61 & 62 & 63 & 64 \\
\hline
\end{tabular}

$\underline{\underline{73}}$

The following item identification prefixes are not wattage limited. Items with the same prefix can be mixed together without problems regarding the isotopic vaues:

\begin{tabular}{llll} 
A-* & BH-* & CAF-* & CCS-* \\
\hline CCT-* & PZ-* & WEC-* &
\end{tabular}

* is the rest of the item identification number.

Use the following table to determine acceptable mass limits for individual items produced during thermal stabilization of oxide items. The last column on the right contains the amount of Putrom any run that will give 15 watts/item, which is the storage vault specification limit. If the data is missing then request isotopic analysis of the items be performed before the items are transferred to $234-5 \mathrm{Z}$ for processing. The STA or Cog. Engineer will then use the isotopic information into calculatea spreadshee and will provide the amount inforial needed for product can loading.

\begin{tabular}{|c|c|c|c|c|c|c|c|c|}
\hline Isotope & $\mathrm{Pu}-238$ & $\mathrm{Pu}-239$ & $\mathrm{Pu}-240$ & $\mathrm{Pu}-241$ & $\mathrm{Pu}-242$ & Am-241 & & \\
\hline Watts/gram & 0.56757 & 0.0019288 & 0.0070824 & 0.1142 & 0.0001159 & 0.1142 & & \\
\hline Run No. & $\% \mathrm{Pu}-238$ & $\% \mathrm{Pu}-239$ & $\%$ Pu-240 & $\% \mathrm{Pu}-241$ & $\% \mathrm{Pu}-242$ & \%Am-241 & Watts $/ \mathrm{kg}$. & grams/pkg. \\
\hline $6-84-12-484$ & & 89.26 & 10.74 & & & & 2.5 & 6042.8 \\
\hline $6-84-12-548$ & & 89.04 & 10.96 & & & & 2.5 & 6015.3 \\
\hline $6-85-01-04$ & & 89.06 & 10.94 & & & & 2.5 & 6017.8 \\
\hline $6-85-01-05$ & & 89.97 & 10.03 & & & & 2.4 & 6133.2 \\
\hline $6-85-01-70$ & & 88.5 & 11.5 & & & & 2.5 & 5948.9 \\
\hline $6-85-01-73$ & & 88.5 & 11.5 & & & & 2.5 & 5948.9 \\
\hline $6-86-01-66$ & & 88.54 & 11.46 & & & & 2.5 & 5953.8 \\
\hline $67-77-03-308$ & & 82 & 18 & & & & 2.9 & 5251.3 \\
\hline $67-77-03-309$ & & $\underline{82}$ & $\underline{18}$ & & & & 2.9 & 5251.3 \\
\hline $67-77-03-310$ & & 82 & 18 & & & & 2.9 & 5251.3 \\
\hline $67-77-03-311$ & & 82 & 18 & & & & 2.9 & 5251.3 \\
\hline $67-77-03-312$ & & 82 & 18 & & & & 2.9 & 5251.3 \\
\hline
\end{tabular}




\begin{tabular}{|l|l|l|}
\hline $\begin{array}{l}\text { PFP THERMAL } \\
\text { STABILIZATION }\end{array}$ & BLEND PLAN & $\begin{array}{l}\text { HNF-4989, REV.1 } \\
\text { PAGE 7 OF 51 }\end{array}$ \\
\hline
\end{tabular}

\begin{tabular}{|c|c|c|c|c|c|c|c|c|}
\hline Isotope & $\mathrm{Pu}-238$ & Pu-239 & $\mathrm{Pu}-240$ & $\mathrm{Pu}-241$ & $\mathrm{Pu}-242$ & Am-241 & & \\
\hline Watts/gram & 0.56757 & 0.0019288 & 0.0070824 & 0.1142 & 0.0001159 & 0.1142 & & \\
\hline Run No. & $\% \mathrm{Pu}-238$ & $\% \mathrm{Pu}-239$ & $\% \mathrm{Pu}-240$ & $\% \mathrm{Pu}-241$ & $\% \mathrm{Pu}-242$ & $\% A m-241$ & Watts $/ \mathrm{kg}$ & grams/pkg. \\
\hline 67-77-03-313 & & 82 & $\underline{18}$ & & & & 2.9 & 5251.3 \\
\hline $67-77-03-314$ & & 82 & 18 & & & & 2.9 & 5251.3 \\
\hline $67-77-03-316$ & & 82 & 18 & & & & 2.9 & 5251.3 \\
\hline $67-77-03-317$ & & 82 & 18 & & & & 2.9 & 5251.3 \\
\hline 67-77-03-318 & & 82 & 18 & & & & $\underline{2.9}$ & 5251.3 \\
\hline $67-77-03-319$ & & 82 & 18 & & & & 2.9 & 5251.3 \\
\hline $67-77=03-320$ & & 82 & 18 & & & & $\underline{2.9}$ & 5251.3 \\
\hline $74-88-12-367$ & & 91.763 & 8.237 & & & & 2.4 & 6374.0 \\
\hline $16-X-5-6$ & & 94 & 6 & & & & $\underline{2.2}$ & 6702.4 \\
\hline $83-X-2-16$ & & 84 & 16 & & & & 2.8 & 5447.9 \\
\hline $204-X-284-12$ & & 88 & 12 & & & & 2.5 & 5888.7 \\
\hline STD-208-X-100-6 & & 94 & 6 & & & & 2.2 & 6702.4 \\
\hline $209-X-249-6$ & & 94 & $\underline{6}$ & & & & 2.2 & 6702.4 \\
\hline $214-X-25-8$ & & 92 & 8 & & & & 2.3 & 6407.3 \\
\hline $215-X-50-8$ & & 92 & $\underline{8}$ & & & & 2.3 & 6407.3 \\
\hline $217-X-247-8$ & & 92 & 8 & & & & 2.3 & 6407.3 \\
\hline $222-X-025-10$ & & 90 & $\underline{10}$ & & & & 2.4 & 6137.1 \\
\hline STD-225-X-243-10 & & 90 & 10 & & & & 2.4 & 6137.1 \\
\hline $230-X-23-12$ & & 88 & 12 & & & & 2.5 & 5888.7 \\
\hline $232-X-101-12$ & & 88 & 12 & & & & 2.5 & 5888.7 \\
\hline STD-259-X-168-24 & & 76 & $\underline{24}$ & & & & 3.2 & 4738.3 \\
\hline $261-X-221-12$ & & 88 & 12 & & & & 2.5 & 5888.7 \\
\hline $\mathrm{GE}-\mathrm{QC}-10$ & & 88.5 & 11.5 & & & & 2.5 & 5948.9 \\
\hline GE-QC-14 & & 88.5 & 11.5 & & & & 2.5 & 5948.9 \\
\hline $\mathrm{GE}-\mathrm{QC}-17$ & & $\underline{88.5}$ & $\underline{11.5}$ & & & & 2.5 & $\underline{5948.9}$ \\
\hline $\mathrm{GE}-\mathrm{QC}-18$ & & 88.5 & 11.5 & & & & $\overline{2.5}$ & 5948.9 \\
\hline GE-QC-20 & & 88.5 & 11.5 & & & & 2.5 & 5948.9 \\
\hline GE-QC-21 & & 88.5 & 11.5 & & & & 2.5 & 5948.9 \\
\hline GE-QC-22 & & 88.5 & $\underline{11.5}$ & & & & 2.5 & 5948.9 \\
\hline GE-QC-23 & & 88.5 & 11.5 & & & & 2.5 & 5948.9 \\
\hline GE-QC-24 & & $\underline{88}$ & 12 & & & & 2.5 & 5888.7 \\
\hline GE-QC-25 & & 88 & 12 & & & & 2.5 & 5888.7 \\
\hline GE-QC-26 & & 88 & 12 & & & & 2.5 & 5888.7 \\
\hline \multicolumn{9}{|l|}{ PPSL-183 } \\
\hline QC-14 & & 88 & 12 & & & & 2.5 & 5888.7 \\
\hline$Q C-15$ & & 88 & 12 & & & & 2.5 & 5888.7 \\
\hline QC-96-09-001 & $\underline{0.06}$ & 87.344 & 11.901 & 0.597 & 0.098 & 1.54 & $\underline{5.3}$ & 2825.6 \\
\hline $\mathrm{TD}-1 \mathrm{~B}$ & & 94.321 & 5.679 & & & & 2.2 & 6752.3 \\
\hline TD-2 & & 94.321 & 5.679 & & & & 2.2 & 6752.3 \\
\hline XDT-148-81-12 & & 78.04 & 18.01 & 3.95 & & & 7.3 & 2057.1 \\
\hline YEB-474-77-01-04 & & $\underline{\underline{83} .5}$ & $\underline{16.5}$ & & & 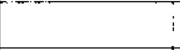 & 2.8 & 5397.3 \\
\hline YEB-474-77-01-05 & & 83.5 & 16.5 & & & & 2.8 & 5397.3 \\
\hline YEC-36-01-023 & 0.068 & 79.128 & 17.344 & 2.772 & $\underline{0.688}$ & & 6.3 & 2378.3 \\
\hline$Y D Z-36-10-01$ & & & & & & & & \\
\hline
\end{tabular}




\begin{tabular}{|l|l|l||}
\hline \hline $\begin{array}{l}\text { PFP THERMAL } \\
\text { STABILIZATION }\end{array}$ & BLEND PLAN & $\begin{array}{l}\text { HNF-4989, REV. 1 } \\
\text { PAGE 8 OF 51 }\end{array}$ \\
\hline
\end{tabular}

\begin{tabular}{|c|c|c|c|c|c|c|c|c|}
\hline Isotope & Pu-238 & Pu-239 & Pu-240 & $\begin{array}{l}\mathrm{Pu}-241 \\
\end{array}$ & $\mathrm{Pu}-242$ & Am-241 & & \\
\hline Watts/gram & 0.56757 & 0.0019288 & 0.0070824 & 0.1142 & 0.0001159 & 0.1142 & & \\
\hline \begin{tabular}{|l|} 
Run No. \\
\end{tabular} & $\% \mathrm{Pu}-238$ & $\% \mathrm{Pu}-239$ & $\% \mathrm{Pu}-240$ & $\% \mathrm{Pu}-241$ & $\% \mathrm{Pu}-242$ & $\% \mathrm{Am}-241$ & Watts $/ \mathrm{kg}$. & grams $/ \mathrm{pkg}$. \\
\hline \multicolumn{9}{|l|}{ YDZ-36-10-02 } \\
\hline YUD-148-81-07 & & 80.23 & 16.67 & 3.1 & & & 6.3 & 2393.0 \\
\hline YUD-148-81-08-01 & & 78.7 & 18.39 & 2.91 & & & 6.1 & 2441.5 \\
\hline YUD-148-81-09 & & 79.58 & 17.51 & 2.91 & & & 6.1 & 2459.7 \\
\hline YUD-148-81-10 & & $\underline{80.33}$ & 16.57 & 3.1 & & & $\underline{6.3}$ & 2395.0 \\
\hline $19 \mathrm{~B} 14-\mathrm{A}$ & & 93.7 & 6.3 & & & & 2.3 & 6656.4 \\
\hline $20-74-07-495$ & & 88.5 & 11.5 & & & & 2.5 & 5948.9 \\
\hline $451-83-09-841$ & & 92 & 8 & & & & 2.3 & 6407.3 \\
\hline $67-74-07-485$ & & 79 & 21 & & & & 3.0 & 4981.6 \\
\hline $102-F-200-6$ & & 94 & 6 & & & & $\overline{2.2}$ & 6702.4 \\
\hline $251 \mathrm{M}-200-2$ & & 97.86 & 2.14 & & & & 2.0 & 7356.2 \\
\hline $253 \mathrm{M}-212-8$ & & 92 & 8 & & & & 2.3 & 6407.3 \\
\hline $254 \mathrm{M}-191-12$ & & 88 & 12 & & & & 2.5 & 5888.7 \\
\hline $256 \mathrm{M}-161-22$ & & 77.65 & 22.35 & & & & 3.1 & 4869.1 \\
\hline $240-\mathrm{N}-1-12$ & & 88 & 12 & & & & 2.5 & 5888.7 \\
\hline $241-\mathrm{N}-2-12$ & & 88 & 12: & & & & 2.5 & 5888.7 \\
\hline $242-\mathrm{N}-1-12$ & & $\underline{88}$ & $\underline{12}$ & & & & 2.5 & $\underline{5888.7}$ \\
\hline $243-\mathrm{N}-5-12$ & & 88 & 12 & & & & 2.5 & 5888.7 \\
\hline $244-\mathrm{N}-7-12$ & & 88 & 12 & & & & 2.5 & $\underline{5888.7}$ \\
\hline $245-\mathrm{N}-10-12$ & & 88 & 12 & & & & 2.5 & 5888.7 \\
\hline $263-\mathrm{N}-2-8$ & & $\underline{92}$ & $\underline{8}$ & & & & 2.3 & 6407.3 \\
\hline $265-\mathrm{N}-5-8$ & & 92 & 8 & & & & 2.3 & 6407.3 \\
\hline $266-N-7-8$ & & 92. & 8 & & & & 2.3 & 6407.3 \\
\hline $39-\mathrm{XA}-100-6$ & & 94 & 6 & & & & 2.2 & 6702.4 \\
\hline $40-\mathrm{XA}-150-6$ & & 94 & 6 & & & & 2.2 & 6702.4 \\
\hline $104-X S-5-6$ & & 94 & 6 & & & & 2.2 & 6702.4 \\
\hline 108-XS-25-6 & & 94 & 6 & & & & 2.2 & 6702.4 \\
\hline $110 \times \mathrm{XS}-38-6$ & & 94 & 6 & & & & 2.2 & 6702.4 \\
\hline $84 \times-2-16$ & & 84 & 16 & & & & 2.8 & 5447.9 \\
\hline $85-X-2-16$ & & 84 & 16 & & & & 2.8 & 5447.9 \\
\hline $86-X-2-16$ & & 84 & $16-$ & & & & 2.8 & 5447.9 \\
\hline $87 \times X-2-16$ & & 84 & 16 & & & & 2.8 & 5447.9 \\
\hline $88-\mathrm{X}-2-16$ & & 84 & 16 & & & & 2.8 & 54479 \\
\hline $89-\mathrm{X}-2-16$ & & 84 & 16 & & & & 2.8 & 5447.9 \\
\hline $90-\mathrm{X}-2-16$ & & 84 & 16 & & & & 2.8 & 5447.9 \\
\hline $91-\mathrm{X}-2-16$ & & 84 & 16 & & & & 2.8 & 5447.9 \\
\hline $92-X-2-16$ & & 84 & 16 & & & & 2.8 & 5447.9 \\
\hline $145-174-X-7-6$ & & 94 & 6 & & & & 2.2 & 6702.4 \\
\hline STD-207-X-50-6 & & 94 & 6 & & & & 2.2 & 6702.4 \\
\hline $216-X-100-8$ & & 92 & 8 & & & & 2.3 & 6407.3 \\
\hline $223-X-50-10$ & & 90 & $\underline{10}$ & & & & 2.4 & 6137.1 \\
\hline $224-\mathrm{X}-100-10$ & & 90 & 10 & & & & 2.4 & 6137.1 \\
\hline $231-X-51-12$ & & 88 & 12 & & & & 2.5 & 5888.7 \\
\hline $90-75-08-677$ & 0.397 & 73.579 & 22.947 & 1.518 & 1.559 & & 7.0 & 2132.8 \\
\hline
\end{tabular}




\begin{tabular}{|l|l|l||}
\hline $\begin{array}{l}\text { PFP THERMAL } \\
\text { STABILIZATION }\end{array}$ & BLEND PLAN & $\begin{array}{l}\text { HNF-4989, REV. 1 } \\
\text { PAGE 9 OF 51 }\end{array}$ \\
\hline
\end{tabular}

\begin{tabular}{|c|c|c|c|c|c|c|c|c|}
\hline Isotope & Pu-238 & Pu-239 & Pu-240 & Pu-241 & Pu-242 & $\mathrm{Am}-241$ & & \\
\hline \begin{tabular}{|l|} 
Watts/gram \\
\end{tabular} & 0.56757 & 0.0019288 & 0.0070824 & 0.1142 & 0.0001159 & 0.1142 & & \\
\hline $\begin{array}{l}\text { Run No. } \\
\end{array}$ & $\% \mathrm{Pu}-238$ & \%Pu-239 & $\% \mathrm{Pu}-240$ & \%Pu-241 & $\% \mathrm{Pu}-242$ & $\% \mathrm{Am}-241$ & Watts $/ \mathrm{kg}$. & grams/pkg. \\
\hline $90-75-08-680$ & 0.0722 & 74.7473 & 23.74482 & 0.8925 & 0.54318 & & 4.6 & 3294.5 \\
\hline $90-75-08-687$ & & 76 & 24 & & & & 3.2 & 4738.3 \\
\hline $\mathrm{A}-28-1.37$ & & 92.54 & 7.46 & & & & 2.3 & 6484.4 \\
\hline AM-3H-01 SWEEP & & 90 & 10 & & & & 2.4 & 6137.1 \\
\hline AO-31-28 SWEEP & & 90 & 10 & & & & 2.4 & 6137.1 \\
\hline AS- $31 \mathrm{H}-57$ SWEEP & & 90 & 10 & & & & 2.4 & 6137.1 \\
\hline CE-5-203-8 & & 76 & 24 & & & & 3.2 & 4738.3 \\
\hline CE-6-265-8 & & 82 & 18 & & & & 2.9 & 5251.3 \\
\hline CZA-465-72-08 & & $\underline{95}$ & 5 & & & & $\underline{2.2}$ & $\underline{6860.3}$ \\
\hline CZA-465-72-23 & & 95 & 5 & & & & $\overline{2.2}$ & 6860.3 \\
\hline CZA-465-72-24 & & 95 & $\underline{5}$ & & & & 2.2 & 6860.3 \\
\hline CZA-465-72-25 & & 95 & 5 & & & & 2.2 & 6860.3 \\
\hline CZA-469-74-228 & & 59.46 & 40.54 & & & & 4.0 & 3733.1 \\
\hline EAB-3-2-1 $\quad$ PART & & 93.5 & 6.5 & & & & 2.3 & 6626.1 \\
\hline EF-1-S SWEEPS & & 91.5 & 8.5 & & & & 2.4 & 6337.5 \\
\hline GEO-30-08-001 & & 80.5 & 19.5 & & & & 2.9 & 5112.9 \\
\hline GE-QC-27 & & $\underline{88}$ & $\underline{12}$ & & & & 2.5 & $\underline{5888.7}$ \\
\hline HUA-40-10-01 & 0.56 & 72.6 & 19.43 & 5.49 & 1.92 & & $\overline{12.2}$ & 1226.8 \\
\hline HUA-40-10-02 & 0.56 & 72.6 & 19.43 & 5.49 & 1.92 & & 12.2 & 1226.8 \\
\hline HUA-40-10-03 & 0.56 & 72.6 & 19,43 & 5.49 & 1.92 & & 12.2 & 1226.8 \\
\hline HUA-40-10-04 & 0.56 & $\underline{72.6}$ & 19.43 & 5.49 & 1.92 & & 12.2 & 1226.8 \\
\hline S-22S-2 & & 90.105 & 9.895 & & & & 2.4 & 6150.7 \\
\hline ZLS-588-75-81 & & 93 & 7 & & & & 2.3 & 6551.5 \\
\hline $10-89-07-84$ & & 94.5 & 5.5 & & & & 2.2 & 6780.4 \\
\hline $10-89-07-88$ & & 94.5 & 5.5 & & & & 2.2 & 6780.4 \\
\hline 22260 & 0.06 & 81.8 & 16.46 & 1.32 & 0.36 & 0.02 & 4.6 & 3250.4 \\
\hline 22277 & 0.06 & 82.18 & 16.24 & 1.17 & $\underline{\underline{0.35}}$ & 0.02 & 4.4 & 3382.0 \\
\hline $6-85-01-46$ & & 88.69 & 11.31 & & & & 2.5 & 5972.1 \\
\hline $6-85-01-85$ & & 88.77 & 11.23 & & & & 2.5 & $\underline{5981.9}$ \\
\hline $6-85-02-131$ & & 89.33 & 10.67 & & & & 2.5 & 6051.6 \\
\hline ACO-29-12-001 S & 0.172 & 88.439 & 9.722 & 1.512 & 0.155 & & 5.1 & 2942.6 \\
\hline$B 707$ & & 94.5 & 5.5 & & & & 2.2 & 6780.4 \\
\hline CZA-001 & & $\underline{81.566}$ & 15.7 & 2.734 & & & 5.8 & 2582.9 \\
\hline $\mathrm{GE}-\mathrm{QC}-\mathrm{Z}$ & & 88.5 & 11.5 & & & & 2.5 & 5948.9 \\
\hline $\mathrm{GE}-\mathrm{OC}-\overline{\mathrm{g}}$ & & 88.5 & 11.5 & & & & 2.5 & 5948.9 \\
\hline $\mathrm{GE}-\mathrm{QC}-9$ & & 88.5 & 11.5 & & & & 2.5 & 5948.9 \\
\hline GES-33-06-001 & 0.104 & 76.492 & 19.769 & 3.54 & 0.095 & & 7.5 & 1997.7 \\
\hline K412 & & 94.5 & 5.5 & & & & 2.2 & 6780.4 \\
\hline NX-29-02-S63-31 & & 74.676 & 20.999 & 4.325 & & & 7.9 & 1906.8 \\
\hline NX-29-02-S63-32 & & 74.676 & 20.999 & 4.325 & & & 7.9 & 1906.8 \\
\hline $\mathrm{NX}-29-03-\mathrm{S} 56-27$ & & 74.676 & 20.999 & 4.325 & & & $\underline{7.9}$ & $\underline{1906.8}$ \\
\hline $\mathrm{NX}-29-03-\mathrm{S} 56-28$ & & 74.676 & 20.999 & 4.325 & & & 7.9 & 1906.8 \\
\hline NX-29-03-S56-29 & & 74.676 & 20.999 & 4.325 & & & 7.9 & 1906.8 \\
\hline $0-30-03-4-5$ & & 86.164 & 11.817 & 2.019 & & & 4.8 & 3122.0 \\
\hline
\end{tabular}




\begin{tabular}{|l|l|l|}
\hline \hline $\begin{array}{l}\text { PFP THERMAL } \\
\text { STABILIZATION }\end{array}$ & BLEND PLAN & $\begin{array}{l}\text { HNF-4989, REV. 1 } \\
\text { PAGE 10 OF 51 }\end{array}$ \\
\hline
\end{tabular}

\begin{tabular}{|c|c|c|c|c|c|c|c|c|}
\hline Isotope & $\mathrm{Pu}-238$ & $\mathrm{Pu}-239$ & $\mathrm{Pu}-240$ & Pu-241 & $\mathrm{Pu}-242$ & Am-241 & & \\
\hline Watts/gram & 0.56757 & 0.0019288 & 0.0070824 & 0.1142 & 0.0001159 & 0.1142 & & \\
\hline Run No. & $\% \mathrm{Pu}-238$ & $\% \mathrm{Pu}-239$ & $\% \mathrm{Pu}-240$ & $\% \mathrm{Pu}-241$ & $\% \mathrm{Pu}-242$ & $\%$ Am-241 & Watts $/ \mathrm{kg}$. & grams $/ \mathrm{pkg}$. \\
\hline $0-30-09-001 \quad C$ & & 86.164 & 11.817 & 2.019 & & & 4.8 & 3122.0 \\
\hline \multicolumn{9}{|l|}{ PPSL-102 } \\
\hline \multicolumn{9}{|l|}{ PPSL-103 } \\
\hline PPSL-104 & & & & & & & & \\
\hline \multicolumn{9}{|l|}{ PPSL-225 } \\
\hline WBO-30-10-1-2 & & 86.674 & 11.592 & 1.734 & & & 4.5 & 3353.5 \\
\hline $\begin{array}{l}\text { ZPS-28-09-049-40- } \\
\mathrm{A}\end{array}$ & & 84.8 & 12.9 & 2.3 & & & $\underline{5.2}$ & 2898.1 \\
\hline ZPS-28-12-019-19 & & $\underline{84.8}$ & $\underline{12.9}$ & 2.3 & & & 5.2 & 2898.1 \\
\hline $\begin{array}{l}\text { ZYP-30-09-001- } \\
0001\end{array}$ & & 81.947 & 13.4 & 4.653 & & & 78 & 1912.4 \\
\hline $\begin{array}{l}\text { ZYP-30-09-001- } \\
0002\end{array}$ & & 81.947 & 13.4 & 4.653 & & & 7.8 & 1912.4 \\
\hline \multicolumn{9}{|l|}{ BLO-38-11-004 } \\
\hline \multicolumn{9}{|l|}{ BLO-38-11-010 } \\
\hline BLO-39-10-15 & 0.332 & 78.200 & 16.935 & 3.420 & 1.113 & 1.760 & 10.5 & 1427.4 \\
\hline BLO-39-10-16 & 0.392 & 73.132 & 21.025 & 3.833 & 1.618 & 2.100 & 11.9 & 1260.3 \\
\hline BLO-39-10-17 & 0.391 & 73.013 & 21.106 & 3.848 & 1.642 & 2.080 & 11.9 & 1261.1 \\
\hline BLO-39-10-18 & 0.383 & 73.583 & 20.685 & 3.771 & 1.578 & 2.460 & 12.2 & 1232.0 \\
\hline BLO-39-10-19 & 0.459 & 73.295 & 20.421 & 4.164 & 1.661 & 2.280 & 12.8 & 1169.5 \\
\hline BLO-39-10-20 & 0.687 & 75.333 & 17.202 & 5.234 & 1.544 & 2.150 & 15.0 & 999.7 \\
\hline BLO-39-10-021 & 0.769 & 76.047 & 15.954 & 5.670 & 1.560 & 3.160 & 17.0 & 879.9 \\
\hline BLO-39-11-01 & 0.675 & 76.341 & 16.149 & 5.287 & 1.548 & 3.160 & 16.1 & 931.9 \\
\hline BLO-39-11-02 & 0.702 & 76.057 & 16.160 & 5.482 & 1.599 & 3.190 & 16.5 & 909.0 \\
\hline BLO-39-11-03 & 0.780 & 76.199 & 15.723 & 5.716 & 1.582 & 3.240 & 17.2 & 870.1 \\
\hline BLO-39-11-04 & 0.837 & 76.417 & 15.301 & 5.907 & 1.538 & 3.280 & 17.8 & 842.6 \\
\hline BLO-39-11-05 & 0.807 & 76.458 & 15.244 & 5.954 & 1.537 & 3.220 & 17.6 & 851.6 \\
\hline BLO-39-11-06 & 0.862 & 76.431 & 15.155 & 6.015 & 1.537 & 3.330 & 18.1 & 828.1 \\
\hline BLO-39-11-07 & 0.868 & 76.385 & 15.142 & 6.071 & 1.534 & 3.310 & 18.2 & 824.8 \\
\hline BLO-39-11-08 & 0.954 & 74.624 & 16.128 & 6.452 & 1.842 & 3.060 & 18.9 & 795.3 \\
\hline BLO-39-11-09 & 1.034 & 74.330 & 16.163 & 6.563 & 1.910 & 3.190 & 19.6 & 765.8 \\
\hline BLO-39-11-10 & 1.005 & 74.594 & 16.056 & 6.470 & 1.875 & 3.120 & 19.2 & 779.9 \\
\hline BLO-39-11-11 & 1.024 & 74.242 & 16.274 & 6.552 & 1.908 & 2.770 & 19.0 & 787.6 \\
\hline BLO-39-11-12 & 1.009 & 74.064 & 16.433 & 6.562 & 1.932 & 3.120 & 19.4 & 774.1 \\
\hline BLO-39-11-13 & 1.010 & 73.887 & 16.569 & 6.563 & 1.971 & 2.800 & 19.0 & 788.4 \\
\hline BLO-39-11-14 & 0.990 & 74.004 & 16.534 & 6.525 & 1.947 & 3.200 & 19.3 & 776.2 \\
\hline BLO-39-11-15 & 0.966 & 74.349 & 16.422 & 6.373 & 1.890 & 3.170 & 19.0 & 790.3 \\
\hline BLO-39-11-16 & 0.410 & 51.878 & 36.453 & 7.181 & 4.078 & 4.020 & 18.7 & 801.9 \\
\hline BLO-39-11-17 & 0.285 & 47.395 & 40.540 & 7.306 & 4.474 & 4.050 & 18.4 & 816.3 \\
\hline \multicolumn{9}{|l|}{ BO-28-01-001-04 } \\
\hline \multicolumn{9}{|l|}{ BO-28-01-001-05 } \\
\hline \multicolumn{9}{|l|}{ BO-28-01-001-06 } \\
\hline \multicolumn{9}{|l|}{ BO-28-01-001-07 } \\
\hline BO-28-01-002-04 & & & & & & & & \\
\hline
\end{tabular}




\begin{tabular}{|l|l|l|}
\hline $\begin{array}{l}\text { PFP THERMAL } \\
\text { STABILIZATION }\end{array}$ & BLEND PLAN & $\begin{array}{l}\text { HNF-4989, REV. } 1 \\
\text { PAGE 11 OF 51 }\end{array}$ \\
\hline
\end{tabular}

\begin{tabular}{|c|c|c|c|c|c|c|c|c|}
\hline Isotope & Pu-238 & Pu-239 & $\mathrm{Pu}-240$ & $\mathrm{Pu}-241$ & $\mathrm{Pu}-242$ & Am-241 & & \\
\hline Watts/gram & 0.56757 & 0.0019288 & 0.0070824 & 0.1142 & 0.0001159 & 0.1142 & & \\
\hline Run No. & $\% \mathrm{Pu}-238$ & $\% \mathrm{Pu}-239$ & $\% \mathrm{Pu}-240$ & $\% \mathrm{Pu}-241$ & $\% \mathrm{Pu}-242$ & $\% \mathrm{Am}-241$ & Watts $/ \mathrm{kg}$. & grams $/$ pkg \\
\hline \multicolumn{9}{|l|}{ BO-29-02-S63-30 } \\
\hline BO-29-03-058-B & 0.093 & 76.410 & 21.400 & 1.991 & 0.106 & 2.365 & 8.5 & 1766.4 \\
\hline BO-30-10-001-A & 0.359 & 77.005 & 18.418 & 3.255 & 0.963 & 3.790 & 12.9 & 1165.1 \\
\hline \multicolumn{9}{|l|}{ BO-30-10-001-B } \\
\hline BO-30-10-002-B & 0.359 & 81.530 & 14.447 & 3.249 & 0.415 & 4.320 & 13.3 & 1129.6 \\
\hline \multicolumn{9}{|l|}{ BO-30-10-003-A } \\
\hline BO-30-10-003-B & 0.364 & 81.310 & 14.680 & 3.203 & 0.443 & 4.321 & 13.3 & 1130.8 \\
\hline BO-30-10-004-B & 0.368 & 77.168 & 18.480 & 3.892 & 0.092 & 3.577 & 13.4 & 1117.9 \\
\hline BO-30-10-005-1 & 0.386 & 80.210 & 15.400 & 3.490 & 0.514 & 4.094 & 13.5 & 1112.1 \\
\hline BO-30-10-005-2 & 0.354 & 79.440 & 17.234 & 1.999 & 0.975 & 5.569 & 13.4 & 1119.0 \\
\hline BO-30-10-006-1 & 0.371 & 79.450 & 16.550 & 2.968 & 0.661 & 4.463 & 13.3 & 1128.0 \\
\hline BO-30-10-006-2 & 0.354 & 79.700 & 16.989 & 1.992 & 0.960 & 5.559 & 13.4 & 1121.4 \\
\hline BO-30-10-007-1 & 0.388 & 80.370 & 15.190 & 3.558 & 0.494 & 4.047 & 13.5 & 1110.0 \\
\hline BO-30-10-007-2 & 0.371 & 79.160 & 16.953 & 2.534 & 0.983 & 5.001 & 13.4 & 1115.9 \\
\hline BO-30-10-008-1 & 0.357 & 78.010 & 17.960 & 3.576 & 0.097 & 3.768 & 13.2 & 1137.4 \\
\hline BO-30-10-008-2 & 0.384 & 79.880 & 15.910 & 3.755 & 0.072 & 3.839 & 13.5 & 1109.7 \\
\hline \multicolumn{9}{|l|}{ BO-30-11-012-1 } \\
\hline BO-30-11-012-2 & 0.595 & 77.113 & 16.408 & 4.381 & 1.503 & 3.797 & 15.4 & 976.0 \\
\hline BO-30-11-013-1 & 0.506 & 79.210 & 16.020 & 4.183 & 0.082 & 4.297 & 15.2 & 985.7 \\
\hline BO-30-11-013-2 & 0.491 & 77.910 & 17.420 & 4.097 & 0.082 & 4.108 & 14.9 & 1007.2 \\
\hline \multicolumn{9}{|l|}{ BO-30-11-014 } \\
\hline BO-30-11-043-1 & 0.227 & 82.120 & 14.990 & 2.207 & 0.456 & 2.698 & 9.5 & 1573.2 \\
\hline BO-30-11-043-2 & 0.218 & 81.040 & 16.330 & 1.753 & 0.660 & 3.314 & 9.7 & 1539.8 \\
\hline BO-30-11-044-1 & 0.439 & 79.480 & 16.250 & 3.208 & 0.623 & 4.602 & 14.1 & 1064.0 \\
\hline BO-30-11-044-2 & 0.473 & 79.510 & 15.860 & 3.576 & 0.581 & 4.173 & 14.2 & 1056.9 \\
\hline BO-30-11-045-1 & 0.453 & 80.180 & 15.400 & 3.447 & 0.521 & 3.950 & 13.7 & 1098.6 \\
\hline BO-30-11-045-2 & 0.426 & 78.820 & 16.690 & 3.384 & 0.680 & 3.889 & 13.4 & 1117.4 \\
\hline BO-30-11-046-1 & 0.490 & 80.910 & 14.550 & 3.582 & 0.428 & 4.685 & 14.8 & 1012.7 \\
\hline BO-30-11-046-2 & 0.505 & 78.630 & 16.350 & 3.865 & 0.650 & 4.506 & 15.1 & 993.1 \\
\hline BO-30-11-047-1 & 0.585 & 77.095 & 16.982 & 3.744 & 1.593 & 4.681 & 15.6 & 959.4 \\
\hline \multicolumn{9}{|l|}{ BO-30-11-047-2 } \\
\hline \multicolumn{9}{|l|}{ BO-30-11-047-3 } \\
\hline BO-30-11-048-1 & 0.468 & 78.140 & 17.370 & 3.934 & 0.088 & 3.814 & 14.2 & 1053.1 \\
\hline BO-30-11-048-2 & 0.471 & 78.650 & 16.810 & 3.977 & 0.092 & 3.745 & 14.2 & 1056.4 \\
\hline BO-30-11-049 & 0.455 & 76.180 & 19.260 & 4.011 & 0.094 & 4.059 & 14.6 & 1025.2 \\
\hline \multicolumn{9}{|l|}{ BO-30-11-11-1 } \\
\hline BO-30-11-11-2 & 0.967 & 71.041 & 21.906 & 4.183 & 1.903 & 4.595 & 18.4 & 813.6 \\
\hline \multicolumn{9}{|l|}{ BO-30-11-11-3 } \\
\hline DZO-35-09-002 & 0.419 & 73.590 & 22.640 & 3.236 & 0.115 & 1.841 & 11.2 & 1339.3 \\
\hline \multicolumn{9}{|l|}{ DZO-35-09-003 } \\
\hline \multicolumn{9}{|l|}{ DZO-35-09-004 } \\
\hline \multicolumn{9}{|l|}{ DZO-35-09-005 } \\
\hline DZO-35-09-006 & 0.434 & 72.820 & 23.180 & 3.449 & 0.117 & 1.808 & 11.5 & 1302.8 \\
\hline
\end{tabular}




\begin{tabular}{|l|c|l||}
\hline $\begin{array}{l}\text { PFP THERMAL } \\
\text { STABILIZATION }\end{array}$ & BLEND PLAN & $\begin{array}{l}\text { HNF-4989, REV. 1 } \\
\text { PAGE 12 OF 51 }\end{array}$ \\
\hline
\end{tabular}

\begin{tabular}{|c|c|c|c|c|c|c|c|c|}
\hline Isotope & $\begin{array}{l}\mathrm{Pu}-238 \\
\end{array}$ & $\mathrm{Pu}-239$ & Pu-240 & Pu-241 & Pu-242 & Am-241 & & \\
\hline \begin{tabular}{|l|} 
Watts/gram \\
\end{tabular} & 0.56757 & 0.0019288 & 0.0070824 & 0.1142 & 0.0001159 & 0.1142 & & \\
\hline Run No. & $\% \mathrm{Pu}-238$ & \%Pu-239 & $\%$ Pu-240 & $\% \mathrm{Pu}-241$ & $\% \mathrm{Pu}-242$ & \%Am-241 & Watts $/ \mathrm{kg}$. & grams/pkg \\
\hline DZO-35-09-007 & 0.444 & 72.878 & 21.868 & 3.011 & 1.800 & 2.034 & 11.2 & 1334.9 \\
\hline \multicolumn{9}{|l|}{ DZO-35-09-008 } \\
\hline \multicolumn{9}{|l|}{ DZO-35-09-009 } \\
\hline \multicolumn{9}{|l|}{ DZO-35-09-010 } \\
\hline \multicolumn{9}{|l|}{ DZO-35-09-011 } \\
\hline \multicolumn{9}{|l|}{ DZO-35-09-013 } \\
\hline \multicolumn{9}{|l|}{ DZO-35-09-015 } \\
\hline \multicolumn{9}{|l|}{ DZO-35-09-016 } \\
\hline \multicolumn{9}{|l|}{ DZO-35-09-019 } \\
\hline DZO-35-09-020 & 0.440 & 73.048 & 21.675 & 3.093 & 1.744 & 2.345 & 11.7 & 1287.2 \\
\hline DZO-35-09-021 & 0.425 & 74.386 & 20.742 & 2.972 & 1.474 & 2.498 & 11.6 & 1296.8 \\
\hline \multicolumn{9}{|l|}{ DZO-35-09-022 } \\
\hline DZO-35-09-023 & 0.427 & 74.055 & 21.871 & 3.538 & 0.109 & 1.994 & 11.7 & 1280.0 \\
\hline \multicolumn{9}{|l|}{ DZO-35-09-024 } \\
\hline \multicolumn{9}{|l|}{ DZO-35-09-025 } \\
\hline DZO-35-09-026 & 0.398 & 74.402 & 21.731 & 3.357 & 0.112 & 1.974 & 11.3 & 1324.8 \\
\hline \multicolumn{9}{|l|}{ DZO-35-09-027 } \\
\hline \multicolumn{9}{|l|}{ DZO-35-09-028 } \\
\hline \multicolumn{9}{|l|}{ DZO-35-09-029 } \\
\hline \multicolumn{9}{|l|}{ DZO-35-09-030 } \\
\hline \multicolumn{9}{|l|}{ DZO-35-09-031 } \\
\hline DZO-35-09-032 & 0.409 & 75.120 & 21.090 & 3.270 & 0.112 & 2.107 & 11.4 & 1315.6 \\
\hline \multicolumn{9}{|l|}{ DZO-35-09-033 } \\
\hline \multicolumn{9}{|l|}{ DZO-35-09-034 } \\
\hline \multicolumn{9}{|l|}{ DZO-35-09-035 } \\
\hline DZO-35-09-036 & 0.384 & 74.110 & 22.240 & 3.162 & 0.104 & 2.155 & 11.3 & 1332.9 \\
\hline \multicolumn{9}{|l|}{ DZO-35-09-037 } \\
\hline \multicolumn{9}{|l|}{ DZO-35-09-038 } \\
\hline \multicolumn{9}{|l|}{ DZO-35-09-039 } \\
\hline DZO-35-09-040 & & & & & & & & \\
\hline DZO-35-09-041 & & & & & & & & \\
\hline DZO-35-09-042 & & & & & & & & \\
\hline DZO-35-09-043 & & & & & & & & \\
\hline DZO-35-09-044 & 0.412 & 73.091 & 22.990 & 3.394 & 0.113 & 2.021 & 11.6 & 1297.5 \\
\hline DZO-35-09-045 & & & & & & & & \\
\hline DZO-35-09-046 & & & & & & & & \\
\hline DZO-35-09-047 & & & & & & & & \\
\hline \begin{tabular}{|l|l|} 
DZO-35-09-048 \\
\end{tabular} & 0.400 & 74.631 & 21.420 & 3.435 & 0.113 & 1.964 & 11.4 & 1316.9 \\
\hline DZO-35-09-049 & & & & & & & & \\
\hline DZO-35-09-050 & & & & & & & & \\
\hline DZO-35-09-051 & & & & & & & & \\
\hline DZO-35-09-052 & 0.372 & 75.260 & 20.960 & 3.298 & 0.110 & 1.978 & 11.1 & 1354.7 \\
\hline DZO-35-09-053 & & & & & & & & \\
\hline
\end{tabular}




\begin{tabular}{|l|c|l||}
\hline $\begin{array}{l}\text { PFP THERMAL } \\
\text { STABILIZATION }\end{array}$ & BLEND PLAN & $\begin{array}{l}\text { HNF-4989, REV . 1 } \\
\text { PAGE 13 OF 51 }\end{array}$ \\
\hline
\end{tabular}

\begin{tabular}{|c|c|c|c|c|c|c|c|c|}
\hline Isotope & $\mathrm{Pu}-238$ & $\mathrm{Pu}-239$ & $\mathrm{Pu}-240$ & $\mathrm{Pu}-241$ & $\mathrm{Pu}-242$ & Am-241 & & \\
\hline Watts/gram & 0.56757 & 0.0019288 & 0.0070824 & 0.1142 & 0.0001159 & 0.1142 & & \\
\hline Run No. & $\% \mathrm{Pu}-238$ & $\% \mathrm{Pu}-239$ & $\% \mathrm{Pu}-240$ & $\% \mathrm{Pu}-241$ & $\% \mathrm{Pu}-242$ & $\% \mathrm{Am}-241$ & Watts $/ \mathrm{kg}$. & grams/pkg. \\
\hline DZO-35-09-054 & 0.365 & 74.861 & 21.540 & 3.132 & 0.101 & 2.082 & 11.0 & 1364.3 \\
\hline \multicolumn{9}{|l|}{ DZO-35-09-055 } \\
\hline DZO-35-09-056 & 0.373 & 74,441 & 21.800 & 3.280 & 0.106 & 1.964 & 11.1 & 1353.3 \\
\hline \multicolumn{9}{|l|}{ DZO-35-09-057 } \\
\hline DZO-35-09-058 & 0.373 & 74.341 & 21.950 & 3.231 & 0.105 & 1.915 & 11.0 & 1365.8 \\
\hline DZO-35-09-059 & 0.371 & 75.571 & 20.690 & 3.270 & 0.098 & 2.032 & 11.1 & 1353.1 \\
\hline DZO-35-09-060 & 0.381 & 72.878 & 22.152 & 2.717 & 1.871 & 2.337 & 10.9 & 1374.6 \\
\hline DZO-35-09-061 & 0.390 & 73.716 & 21.394 & 2.853 & 1.647 & 2.285 & 11.0 & 1360.9 \\
\hline DZO-35-09-062 & 0.386 & 73.950 & 22.440 & 1.930 & 1.297 & 3.331 & 11.2 & 1337.1 \\
\hline \multicolumn{9}{|l|}{ DZO-35-09-063 } \\
\hline \multicolumn{9}{|l|}{ DZO-35-09-064 } \\
\hline \multicolumn{9}{|l|}{ DZO-35-09-065 } \\
\hline \multicolumn{9}{|l|}{ DZO-35-09-066 } \\
\hline \multicolumn{9}{|l|}{ DZO-35-09-067 } \\
\hline DZO-35-09-068 & 0.389 & 74.530 & 21.590 & 3.385 & 0.106 & 1.968 & 11.3 & 1328.8 \\
\hline \multicolumn{9}{|l|}{ DZO-35-09-069 } \\
\hline \multicolumn{9}{|l|}{ DZO-35-09-070 } \\
\hline \multicolumn{9}{|l|}{ DZO-35-09-071 } \\
\hline \multicolumn{9}{|l|}{ DZO-35-09-072 } \\
\hline \multicolumn{9}{|l|}{ DZO-35-09-073 } \\
\hline DZO-35-09-074 & 0.364 & 74.500 & 22.226 & 1.657 & 1.255 & 3.553 & 11.0 & 1360.3 \\
\hline \multicolumn{9}{|l|}{ DZO-35-09-075 } \\
\hline \multicolumn{9}{|l|}{ DZO-35-09-076 } \\
\hline DZO-35-09-077 & 0.368 & 75.490 & 20.770 & 3.276 & 0.096 & 1.973 & 11.0 & 1362.4 \\
\hline DZO-35-09-078 & 0.392 & 73.561 & 22.540 & 3.392 & 0.115 & 1.876 & 11.3 & 1332.7 \\
\hline \multicolumn{9}{|l|}{ DZO-35-09-079 } \\
\hline DZO-35-09-080 & 0.387 & 73.900 & 22.310 & 3.295 & 0.108 & 2.003 & 11.3 & 1333.1 \\
\hline \multicolumn{9}{|l|}{ DZO-35-09-081 } \\
\hline \multicolumn{9}{|l|}{ DZO-35-09-082 } \\
\hline DZO-35-09-287-A & 0.483 & 69.504 & 24.088 & 3.280 & 2.645 & 4.160 & 14.3 & 1050.0 \\
\hline DZO-35-09-287-B & 0.459 & 74.190 & 21.600 & 3.639 & 0.112 & 4.283 & 14.6 & 1026.4 \\
\hline DZO-35-09-294-A & 0.846 & 81.730 & 12.800 & 4.342 & 0.282 & 5.921 & 19.0 & 789.2 \\
\hline DZO-35-09-294-B & 0.835 & 74.671 & 19.320 & 5.080 & 0.094 & 4.358 & 18.3 & 818.6 \\
\hline DZO-35-09-295-A & 0.693 & 80.840 & 14.180 & 4.221 & 0.066 & 4.574 & 16.5 & 906.9 \\
\hline \multicolumn{9}{|l|}{ DZO-35-09-295-B } \\
\hline DZO-35-09-301-A & 0.750 & 78.874 & 15.751 & 4.552 & 0.073 & 4.649 & 17.4 & 862.2 \\
\hline DZO-35-09-301-B & 0.802 & 78.520 & 16.150 & 3.902 & 0.627 & 5.631 & 18.1 & 829.0 \\
\hline DZO-35-09-302-A & 0.869 & 78.914 & 15.355 & 4.330 & 0.531 & 5.274 & 18.5 & 810.3 \\
\hline \multicolumn{9}{|l|}{ DZO-35-09-302-B } \\
\hline DZO-35-09-303-A & 0.797 & 79.241 & 15.200 & 4.685 & 0.077 & 4.752 & 17.9 & 837.8 \\
\hline DZO-35-09-303-B & 0.824 & 77.630 & 16.940 & 4.523 & 0.083 & 4.973 & 18.2 & 823.3 \\
\hline DZO-35-10-025-A & 0.279 & 76.780 & 19.700 & 2.057 & 1.184 & 1.844 & 8.9 & 1682.5 \\
\hline DZO-35-10-026-A & 0.188 & 85.280 & 12.880 & 1.386 & 0.266 & 1.218 & 6.6 & 2273.2 \\
\hline
\end{tabular}




\begin{tabular}{|l|l|l|}
\hline \hline $\begin{array}{l}\text { PFP THERMAL } \\
\text { STABILIZATION }\end{array}$ & BLEND PLAN & $\begin{array}{l}\text { HNF-4989, REV. 1 } \\
\text { PAGE 14 OF 51 }\end{array}$ \\
\hline
\end{tabular}

\begin{tabular}{|c|c|c|c|c|c|c|c|c|}
\hline Isotope & Pu-238 & $\mathrm{Pu}-239$ & $\mathrm{Pu}-240$ & $\mathrm{Pu}-241$ & $\mathrm{Pu}-242$ & Am-241 & & \\
\hline Watts/gram & 0.56757 & 0.0019288 & 0.0070824 & 0.1142 & 0.0001159 & 0.1142 & & \\
\hline Run No. & $\% \mathrm{Pu}-238$ & $\% \mathrm{Pu}-239$ & $\% \mathrm{Pu}-240$ & $\% \mathrm{Pu}-241$ & $\% \mathrm{Pu}-242$ & $\% \mathrm{Am}-241$ & Watts $/ \mathrm{kg}$. & grams/pkg. \\
\hline DZO-35-10-027-A & 0.307 & 73.530 & 22.180 & 2.140 & 1.843 & 2.032 & 9.5 & 1579.6 \\
\hline DZO-35-10-028-A & 0.154 & 80.870 & 16.900 & 1.400 & 67.610 & 1.180 & 6.7 & 2254.0 \\
\hline DZO-35-10-043-A & 0.226 & 85.190 & 12.470 & 1.869 & 0.245 & 3.172 & 9.6 & 1567.8 \\
\hline \multicolumn{9}{|l|}{ DZO-35-10-053-A } \\
\hline DZO-35-10-053-B & 0.277 & 85.410 & 11.890 & 2.356 & 0.067 & 3.130 & 10.3 & 1452.8 \\
\hline DZO-35-10-054-B & 0.392 & 80.649 & 15.990 & 2.884 & 0.085 & 3.691 & 12.4 & 1207.3 \\
\hline DZO-35-10-289-A & 0.656 & 77.670 & 17.490 & 4.104 & 0.081 & 4.790 & 16.6 & 902.7 \\
\hline DZO-35-10-289-B & 0.705 & 73.878 & 20.239 & 3.790 & 1.388 & 4.864 & 16.7 & 895.8 \\
\hline \multicolumn{9}{|l|}{ DZO-35-10-291-A } \\
\hline \multicolumn{9}{|l|}{ DZO-35-10-291-B } \\
\hline DZO-35-10-296-A & 0.712 & 76.370 & 18.560 & 4.267 & 0.090 & 4.393 & 16.7 & 897.1 \\
\hline DZO-35-10-297-A & 0.732 & 76.910 & 17.660 & 4.617 & 0.082 & 4.237 & 17.0 & 882.3 \\
\hline DZO-35-10-297-B & 0.732 & 81.200 & 13.890 & 3.806 & 0.372 & 5.375 & 17.2 & 872.5 \\
\hline DZO-35-10-298-A & 0.693 & 79.330 & 15.470 & 4.430 & 0.077 & 4.554 & 16.8 & 891.8 \\
\hline \begin{tabular}{|l|} 
DZO-35-10-298-B \\
\end{tabular} & 0.698 & 80.531 & 14.200 & 4.507 & 0.063 & 4.716 & 17.1 & 879.4 \\
\hline DZO-35-10-300-A & 0.743 & 77.430 & 17.220 & 4.512 & 0.094 & 4.817 & 17.6 & 853.1 \\
\hline DZO-35-10-300-B & 0.734 & 57.670 & 30.210 & 3.812 & 7.574 & 4.020 & 16.4 & 916.3 \\
\hline LAO-C $* *-226$ & 0.062 & 81.586 & 16.590 & 1.410. & 0.352 & 0.100 & 4.8 & 3108.5 \\
\hline${\mathrm{LAO}-\mathrm{C}^{* *}-227}$ & 0.070 & 81.809 & 16.400 & 1.370 & 0.351 & 0.106 & 4.8 & 3110.0 \\
\hline LAO-C**-228 & 0.058 & 82.254 & 16.000 & 1.360 & 0.328 & 0.095 & 4.7 & 3184.2 \\
\hline LAO-C**-229 & 0.058 & 82.033 & 16.200 & 1.370 & 0.339 & 0.081 & 4.7 & 3180.4 \\
\hline LAO-C**-230 & 0.056 & 82.109 & 16.160 & 1.320 & 0.355 & 0.061 & 4.6 & 3243.9 \\
\hline LAO-C ${ }^{* *}-231$ & 0.060 & 81.656 & 16.490 & 1.430 & 0.364 & 0.068 & 4.8 & 3128.8 \\
\hline LAO-C ${ }^{* *}-232$ & 0.055 & 82.009 & 16.210 & 1.380 & 0.346 & 0.086 & 4.7 & 3180.2 \\
\hline 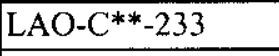 & 0.058 & 81.984 & 16.240 & 1.380 & 0.338 & 0.071 & 4.7 & 3179.4 \\
\hline LAO-C ${ }^{* *}-234$ & 0.067 & 81.980 & 16.250 & 1.360 & 0.343 & 0.085 & 4.8 & 3149.6 \\
\hline $\mathrm{LAO} \mathrm{C}^{* *}-242$ & 0.060 & 82.072 & 16.190 & 1.340 & 0.338 & 0.136 & 4.8 & 3153.9 \\
\hline LA0-C**-245 & 0.062 & 81.876 & 16.360 & 1.350 & 0.352 & 0.144 & 4.8 & 3127.4 \\
\hline LAO-C ${ }^{* *}-247$ & 0.059 & 81.940 & 16.360 & 1.300 & 0.335 & 0.128 & 4.7 & 3187.8 \\
\hline LAO-C**-259 & 0.061 & 81.898 & 16.360 & 1.340 & 0.341 & 0.112 & 4.7 & 3162.7 \\
\hline PBO-45-10-025 & 0.059 & 88.866 & 10.044 & 0.850 & 0.181 & 0.047 & 3.8 & 3962.8 \\
\hline PBO-45-11-032 & 0.112 & 84.129 & 13.397 & 2.086 & 0.276 & 0.006 & 5.6 & 2680.2 \\
\hline PBO-45-11-032C & 0.105 & 84.770 & 12.928 & 1.923 & 0.275 & 0.133 & 5.5 & 2729.0 \\
\hline PBO-45-12-037 & 0.095 & 84.991 & 12.839 & 1.825 & 0.250 & 0.017 & 5.2 & 2889.0 \\
\hline PBO-45-12-038 & 0.115 & 84.824 & 12.687 & 2.114 & 0.260 & 0.009 & 5.6 & 2672.7 \\
\hline PBO-47-09-011 & 0.094 & 86.143 & 12.104 & 1.452 & 0.207 & 0.010 & 4.7 & 3176.8 \\
\hline PBO-47-09-011C & 0.086 & 85.789 & 12.439 & 1.447 & 0.239 & 0.001 & 4.7 & 3208.6 \\
\hline PBO-47-09-012 & 0.091 & 86.137 & 12.107 & 1.458 & 0.207 & 0.008 & 4.7 & 3184.6 \\
\hline PBO-47-09-013 & 0.089 & 86.149 & 12.091 & 1.462 & 0.209 & 0.010 & 4.7 & 3188.8 \\
\hline \multicolumn{2}{|l|}{$* *=01$ through 13} & & & & & & & \\
\hline
\end{tabular}




\begin{tabular}{|l|l|l|}
\hline $\begin{array}{l}\text { PFP THERMAL } \\
\text { STABILIZATION }\end{array}$ & BLEND PLAN & $\begin{array}{l}\text { HNF-4989, REV. } 1 \\
\text { PAGE 15 OF 51 }\end{array}$ \\
\hline
\end{tabular}

\begin{tabular}{|c|c|c|}
\hline \multicolumn{3}{|c|}{ VAULT 1} \\
\hline ITEMID & $\overline{\mathrm{CAT}}$ & COEI \\
\hline ARF-102-85-186-S & 102 & $\mathrm{CO2}$ \\
\hline$A R F-102-85-199$ & 102 & $\mathrm{CO2}$ \\
\hline$A R F-102-85-299$ & 102 & $\mathrm{CO1}$ \\
\hline ARF-102-85-307 & 102 & $\mathrm{CO1}$ \\
\hline$A R F-102-85-308-S$ & 102 & $\mathrm{CO1}$ \\
\hline ARF-102-85-309-S & 102 & $\mathrm{CO} 1$ \\
\hline$A R F-102-85-323$ & 102 & $\mathrm{COI}$ \\
\hline$A R F-102-85-332$ & 102 & $\mathrm{COI}$ \\
\hline $\mathrm{ARF}-102-85-335$ & 102 & $\mathrm{CO}$ \\
\hline ARF-102-85-357 & 102 & $\mathrm{CO}$ \\
\hline ARF-102-85-361 & 102 & $\mathrm{CO1}$ \\
\hline ARF-102-85-364 & 102 & $\mathrm{CO2}$ \\
\hline ARF-102-85-366 & 102 & $\mathrm{CO1}$ \\
\hline ARF $-102-85-374-1$ & 51 & $\mathrm{COO}$ \\
\hline ARF-102-85-374-2 & 51 & $\mathrm{COO}$ \\
\hline$A R F-102-85-381$ & 102 & $\mathrm{CO1}$ \\
\hline $\mathrm{ARF}-102-85-384$ & 102 & $\mathrm{COI}$ \\
\hline$\overline{A R F}-102-85-396$ & 102 & $\mathrm{COI}$ \\
\hline$A R F-102-85-454$ & 102 & $\mathrm{CO}$ \\
\hline ARF - 102-85-538 & 102 & $\mathrm{CO1}$ \\
\hline ARF-102-85-539 & 102 & $\mathrm{CO1}$ \\
\hline$A R F-102-85-868$ & 102 & $\mathrm{CO1}$ \\
\hline $\mathrm{ARF}-102-85-874$ & 102 & $\mathrm{CO1}$ \\
\hline$A R F-102-85-875$ & 102 & $\mathrm{C01}$ \\
\hline ARF-102-85-876 & 102 & $\mathrm{CO1}$ \\
\hline$A R F-102-85-879$ & 102 & $\mathrm{COI}$ \\
\hline ARF-102-85-892 & 102 & $\mathrm{CO1}$ \\
\hline ARF-102-85-904 & 102 & $\mathrm{COI}$ \\
\hline ARF $-102-86-460$ & 102 & $\mathrm{CO1}$ \\
\hline ARF-102-86-493 & 102 & $\mathrm{COI}$ \\
\hline ARF $-102-90-03$ & 102 & $\mathrm{CO1}$ \\
\hline
\end{tabular}




\begin{tabular}{|l|l|l|}
\hline PFP THERMAL & BLEND PLAN & $\begin{array}{l}\text { HNF-4989, REV. 1 } \\
\text { PAGE 16 OF 51 }\end{array}$ \\
\hline
\end{tabular}

\begin{tabular}{|c|c|c|}
\hline \multicolumn{3}{|c|}{ VÄŨLT 1} \\
\hline ITEMID & CAT & COEI \\
\hline $41-79-11-1786$ & 41 & $\mathrm{CO1}$ \\
\hline $41-79-11-1787$ & 41 & $\mathrm{CO} 1$ \\
\hline $41-79-11-1788$ & 41 & $\underline{\mathrm{CO1}}$ \\
\hline $41-85-08-1377$ & 41 & $\mathrm{CO1}$ \\
\hline $41-85-08-1378$ & 42 & $\mathrm{COI}$ \\
\hline $41-85-08-1379$ & 41 & $\mathrm{CO1}$ \\
\hline $41-87-06-251$ & 41 & $\mathrm{COI}$ \\
\hline $42-84-12-513$ & 42 & $\mathrm{CO1}$ \\
\hline $42-85-03-544$ & 42 & $\mathrm{CO1}$ \\
\hline $42-85-03-545$ & 42 & $\mathrm{CO} 1$ \\
\hline $42-86-06-509$ & 42 & $\mathrm{CO1}$ \\
\hline $5-88-07-147$ & $\underline{5}$ & $\mathrm{COO}$ \\
\hline $5-88-08-195$ & 5 & $\mathrm{COO}$ \\
\hline $5-88-10-267$ & 5 & $\mathrm{COO}$ \\
\hline $5-88-10-268$ & 5 & $\mathrm{COO}$ \\
\hline $5-88-11-339$ & 5 & $\mathrm{COO}$ \\
\hline $51-78-06-518$ & 51 & $\mathrm{C01}$ \\
\hline $6-84-12-484$ & 6 & $\mathrm{CO1}$ \\
\hline $6-84-12-548$ & $\underline{6}$ & $\mathrm{CO1}$ \\
\hline $6-85-01-04$ & 6 & $\mathrm{COI}$ \\
\hline $6-85-01-05$ & 6 & $\overline{\mathrm{CO}}$ \\
\hline $6-85-01-70$ & 6 & GOO \\
\hline $6-85-01-73$ & 6 & $\mathrm{CO1}$ \\
\hline $6-86-01-66$ & $\bar{~}$ & $\mathrm{CO1}$ \\
\hline $61-82-09-65$ & 61 & $\mathrm{CO1}$ \\
\hline $61-82-10-142$ & 61 & $\mathrm{COI}$ \\
\hline $61-82-12-565$ & 61 & $\mathrm{CO}$ \\
\hline $61-82-12-566$ & 61 & $\mathrm{CO1}$ \\
\hline $61-82-12-567$ & 61 & $\mathrm{COL}$ \\
\hline $61-82-12-467$ & 61 & $\mathrm{CO1}$ \\
\hline $61-83-01-018$ & 61 & $\mathrm{COI}$ \\
\hline
\end{tabular}




\begin{tabular}{|l|l|l|}
\hline $\begin{array}{l}\text { PFP THERMAL } \\
\text { STABILIZATION }\end{array}$ & BLEND PLAN & $\begin{array}{l}\text { HNF-4989, REV. } \\
\text { PAGE 17 OF 51 }\end{array}$ \\
\hline
\end{tabular}

\begin{tabular}{|c|c|c|}
\hline \multicolumn{3}{|c|}{ VĀULT 1} \\
\hline ITEMID & CAT & COEI \\
\hline $61-83-03-143$ & 61 & $\mathrm{COO}$ \\
\hline $61-83-03-144$ & 61 & $\mathrm{COO}$ \\
\hline $61-83-03-145$ & 61 & $\mathrm{COO}$ \\
\hline $61-83-03-146$ & 61 & $\mathrm{COO}$ \\
\hline $61-83-03-147$ & 61 & $\mathrm{COO}$ \\
\hline $61-83-03-148$ & 61 & $\mathrm{COO}$ \\
\hline $61-83-03-156$ & 61 & $\mathrm{COO}$ \\
\hline $61-83-04-199$ & 61 & $\mathrm{CO1}$ \\
\hline $61-83-04-238$ & 61 & $\mathrm{CO1}$ \\
\hline $61-83-08-568$ & 61 & $\mathrm{CO} 1$ \\
\hline $61-85-02-127$ & 61 & $\mathrm{CO1}$ \\
\hline $61-85-03-338$ & 61 & $\mathrm{CO1}$ \\
\hline $61-85-03-483$ & 61 & $\mathrm{CO} 1$ \\
\hline $61-85-04-629$ & 61 & $\mathrm{CO1}$ \\
\hline $61-85-04-630$ & $\underline{61}$ & $\mathrm{CO1}$ \\
\hline $61-85-05-650$ & 61 & $\mathrm{CO1}$ \\
\hline $61-85-05-713$ & 61 & $\mathrm{CO1}$ \\
\hline $61-85-05-714$ & 61 & $\mathrm{CO} 1$ \\
\hline $61-85-05-715$ & 61 & C01 \\
\hline $61-85-05-716$ & 61 & $\mathrm{COI}$ \\
\hline $61-85-05-821$ & 61 & GOO \\
\hline $61-85-06-940$ & 61 & $\mathrm{COI}$ \\
\hline $61-85-06-941$ & 61 & $\mathrm{CO}$ \\
\hline $61-85-06-942$ & 61 & $\mathrm{CO1}$ \\
\hline $61-85-06-943$ & 61 & $\mathrm{CO1}$ \\
\hline $61-85-06-950$ & 61 & $\mathrm{COI}$ \\
\hline $61-85-06-951$ & 61 & $\underline{\mathrm{CO1}}$ \\
\hline $61-85-06-991$ & $\overline{61}$ & $\mathrm{CO1}$ \\
\hline $61-85-06-1009$ & 61 & $\mathrm{GOO}$ \\
\hline $61-85-06-1010$ & 61 & $\mathrm{COI}$ \\
\hline $61-85-06-1011$ & 61 & $\mathrm{CO1}$ \\
\hline
\end{tabular}




\begin{tabular}{|l|l|l|}
\hline $\begin{array}{l}\text { PFP THERMAL } \\
\text { STABILIZATION }\end{array}$ & BLEND PLAN & $\begin{array}{l}\text { HNF-4989, REV. 1 } \\
\text { PAGE 18 OF 51 }\end{array}$ \\
\hline
\end{tabular}

\begin{tabular}{|c|c|c|}
\hline \multicolumn{3}{|c|}{ VAULT 1} \\
\hline ITEMID & CAT & COEI \\
\hline $61-85-07-1018-1$ & 61 & $\mathrm{COI}$ \\
\hline $61-85-08-1266$ & 61 & $\mathrm{CO1}$ \\
\hline $61-85-08-1298$ & 61 & $\mathrm{CO1}$ \\
\hline $61-85-08-1299$ & 61 & $\mathrm{CO}$ \\
\hline $61-85-08-1326$ & 61 & $\mathrm{CO}$ \\
\hline $61-85-08-1327$ & 61 & $\mathrm{CO}$ \\
\hline $61-85-08-1328$ & 61 & CO1 \\
\hline $61-85-08-1332$ & 61 & $\mathrm{CO1}$ \\
\hline $61-85-08-1344$ & 61 & $\mathrm{CO1}$ \\
\hline $61-85-08-1360$ & 61 & $\mathrm{CO1}$ \\
\hline $61-85-08-1365$ & 61 & $\mathrm{CO1}$ \\
\hline $61-85-08-1366$ & 61 & CO1 \\
\hline $61-85-08-1382$ & 61 & $\mathrm{COI}$ \\
\hline $61-85-09-1383$ & 61 & $\mathrm{CO1}$ \\
\hline $62-83-03-176$ & 62 & $\mathrm{COO}$ \\
\hline $62-85-03-324$ & 62 & $\mathrm{COI}$ \\
\hline $62-85-03-325$ & 62 & $\mathrm{CO} 1$ \\
\hline $62-85-08-1352$ & 62 & $\mathrm{CO} 1$ \\
\hline $62-85-09-1391$ & 62 & $\mathrm{CO1}$ \\
\hline $62-85-09-1392$ & 62 & $\mathrm{CO1}$ \\
\hline $62-85-09-1466$ & 62 & $\mathrm{COI}$ \\
\hline $62-85-10-1653$ & $\overline{62}$ & $\overline{\mathrm{CO1}}$ \\
\hline $62-86-02-82$ & 62 & $\mathrm{CO1}$ \\
\hline $62-86-02-107$ & 62 & $\mathrm{CO1}$ \\
\hline $62-86-02-125$ & 62 & $\mathrm{COI}$ \\
\hline $62-86-03-221$ & 62 & $\mathrm{COI}$ \\
\hline $62-86-03-247$ & 62 & $\mathrm{CO1}$ \\
\hline $62-86-03-249$ & 62 & $\mathrm{CO1}$ \\
\hline $62-87-03-189$ & 62 & $\mathrm{COI}$ \\
\hline $62-87-03-191$ & $\overline{62}$ & $\mathrm{COI}$ \\
\hline $62-87-09-301$ & 62 & $\mathrm{CO1}$ \\
\hline
\end{tabular}




\begin{tabular}{|l|l|l||}
\hline $\begin{array}{l}\text { PFP THERMAL } \\
\text { STABILIZATION }\end{array}$ & BLEND PLAN & $\begin{array}{l}\text { HNF-4989, REV. 1 } \\
\text { PAGE 19 OF 51 }\end{array}$ \\
\hline
\end{tabular}

\begin{tabular}{|c|c|c|}
\hline \multicolumn{3}{|c|}{ VAULT 1} \\
\hline ITEMID & $\mathrm{CAT}$ & COEI \\
\hline $62-88-01-01$ & 62 & $\mathrm{CO1}$ \\
\hline $63-85-02-161$ & 63 & $\mathrm{CO1}$ \\
\hline $63-85-02-166$ & 63 & COI \\
\hline $63-85-02-175$ & 63 & $\mathrm{COI}$ \\
\hline $63-85-02-181$ & 63 & $\mathrm{CO1}$ \\
\hline $63-85-02-182$ & 63 & $\mathrm{CO1}$ \\
\hline $63-85-02-185$ & 63 & $\mathrm{COI}$ \\
\hline $63-85-02-190$ & 63 & $\mathrm{COI}$ \\
\hline $63-85-02-192$ & 63 & $\mathrm{CO1}$ \\
\hline $63-85-02-196$ & 63 & $\mathrm{CO1}$ \\
\hline $63-85-02-197$ & 63 & $\mathrm{CO1}$ \\
\hline $63-85-03-257$ & 63 & $\mathrm{CO1}$ \\
\hline $63-85-03-269$ & 63 & $\mathrm{CO1}$ \\
\hline $63-85-03-319$ & 63 & $\overline{\mathrm{CO}}$ \\
\hline $63-85-03-320$ & 63 & $\mathrm{CO1}$ \\
\hline $63-85-03-321$ & 63 & $\mathrm{CO1}$ \\
\hline $63-85-03-\overline{332-1}$ & 63 & $\mathrm{CO1}$ \\
\hline $63-85-04-595$ & 63 & $\mathrm{COI}$ \\
\hline $63-85-04-582$ & 63 & $\overline{\mathrm{CO}}$ \\
\hline $63-85-04-573$ & 63 & $\mathrm{CO1}$ \\
\hline $63-87-07-\overline{259}$ & 63 & $\overline{\mathrm{CO} 1}$ \\
\hline $63-87-08-275$ & 63 & $\mathrm{CO1}$ \\
\hline $63-87-08-\overline{276}$ & $\underline{\underline{63}}$ & $\underline{\mathrm{CO1}}$ \\
\hline $63-87-08-277$ & $\underline{63}$ & $\mathrm{CO1}$ \\
\hline $63-87-08-284$ & 63 & $\mathrm{COH}$ \\
\hline $63-87-12-341$ & 63 & $\mathrm{CO1}$ \\
\hline $63-88-01-22$ & 63 & $\mathrm{CO1}$ \\
\hline $63-88-05-90$ & 63 & $\mathrm{CO1}$ \\
\hline $63-88-05-92$ & 63 & $\mathrm{CO1}$ \\
\hline $63-88-05-93$ & 63 & $\mathrm{CO1}$ \\
\hline $63-88-06-100$ & 63 & $\mathrm{CO1}$ \\
\hline
\end{tabular}




\begin{tabular}{|l|l|l|}
\hline $\begin{array}{l}\text { PFP THERMAL } \\
\text { STABILIZATION }\end{array}$ & BLEND PLAN & $\begin{array}{l}\text { HNF-4989, REV. 1 } \\
\text { PAGE 20 OF 51 }\end{array}$ \\
\hline
\end{tabular}

\begin{tabular}{|c|c|c|}
\hline \multicolumn{3}{|c|}{ VAULT 1} \\
\hline ITEMID & $\overline{\mathrm{CAT}}$ & COEI \\
\hline $63-88-06-102$ & 63 & $\mathrm{COI}$ \\
\hline $63-88-06-105$ & 63 & $\mathrm{COI}$ \\
\hline $63-88-06-106$ & 63 & $\mathrm{COI}$ \\
\hline $63-88-06-112$ & 63 & $\mathrm{COI}$ \\
\hline $63-88-06-113$ & 63 & $\mathrm{COI}$ \\
\hline $63-88-06-121$ & 63 & $\mathrm{CO} 1$ \\
\hline $63-88-06-122$ & 63 & $\mathrm{CO1}$ \\
\hline $63-88-06-124$ & 63 & $\mathrm{CO1}$ \\
\hline $63-88-07-128$ & 63 & $\mathrm{CO1}$ \\
\hline $63-88-07-130$ & 63 & $\mathrm{CO1}$ \\
\hline $63-88-07-131$ & $\underline{\underline{63}}$ & $\underline{\mathrm{CO} 1}$ \\
\hline $63-88-08-194-1$ & 63 & $\mathrm{CO1}$ \\
\hline $63-88-08-194-2$ & 63 & $\mathrm{CO1}$ \\
\hline $63-88-08-204$ & 63 & $\mathrm{COI}$ \\
\hline $63-88-08-206$ & 63 & $\mathrm{COI}$ \\
\hline $63-88-09-218$ & 63 & $\mathrm{COI}$ \\
\hline $63-88-09-219$ & 63 & $\mathrm{COH}$ \\
\hline $63-88-09-220$ & 63 & $\mathrm{COI}$ \\
\hline $63-88-09-231$ & 63 & $\mathrm{CO2}$ \\
\hline $63-88-09-232$ & 63 & $\mathrm{CO2}$ \\
\hline $63-88-09-233$ & 63 & $\mathrm{CO2}$ \\
\hline $63-88-09-234$ & 63 & $\mathrm{CO2}$ \\
\hline $63-88-10-264$ & 63 & $\underline{\mathrm{COO}}$ \\
\hline $63-88-10-265$ & 63 & $\mathrm{COO}$ \\
\hline $63-88-10-266$ & 63 & $\mathrm{COO}$ \\
\hline $63-88-10-273$ & 63 & $\mathrm{COI}$ \\
\hline $63-88-10-274$ & $\underline{63}$ & Co1 \\
\hline $63-88-10-283$ & $\underline{63}$ & $\mathrm{CO1}$ \\
\hline $63-88-10-281$ & 63 & $\mathrm{CO1}$ \\
\hline $63-88-10-282$ & 63 & $\mathrm{COI}$ \\
\hline $63-88-10-284$ & 63 & $\mathrm{CO1}$ \\
\hline
\end{tabular}




\begin{tabular}{|l|l|l|}
\hline $\begin{array}{l}\text { PFP THERMAL } \\
\text { STABILIZATION }\end{array}$ & BLEND PLAN & $\begin{array}{l}\text { HNF-4989, REV. 1 } \\
\text { PAGE 21 OF 51 }\end{array}$ \\
\hline
\end{tabular}

\begin{tabular}{|c|c|c|}
\hline \multicolumn{3}{|c|}{ VAULT 1} \\
\hline ITEMID & $\overline{\mathrm{CAT}}$ & COEI \\
\hline $63-88-10-298$ & 63 & $\mathrm{CO} 1$ \\
\hline $63-88-11-345$ & 63 & $\mathrm{CO1}$ \\
\hline $63-88-11-347$ & 63 & $\underline{\underline{\mathrm{CO1}}}$ \\
\hline $63-88-12-369$ & 63 & $\overline{\mathrm{CO} 11}$ \\
\hline $63-88-12-370$ & $\underline{63}$ & C01 \\
\hline $63-88-12-374$ & 63 & $\mathrm{CO1}$ \\
\hline $63-88-12-375$ & $\underline{63}$ & $\overline{\mathrm{CO} 01}$ \\
\hline $64-85-10-1593$ & $\underline{64}$ & $\mathrm{CO} 01$ \\
\hline $64-85-10-1594$ & 64 & $\mathrm{CO1}$ \\
\hline $64-85-10-1629$ & 64 & $\mathrm{CO1}$ \\
\hline $64-85-10-1569$ & $\underline{\underline{64}}$ & $\mathrm{CO1}$ \\
\hline $64-85-11-1686$ & 64 & $\overline{\mathrm{CO}}$ \\
\hline $64-85-11-1688$ & 64 & $\mathrm{CO1}$ \\
\hline $64-85-11-1745$ & $\overline{64}$ & $\mathrm{CO1}$ \\
\hline $64-85-11-1746$ & 64 & $\overline{\mathrm{CO1}}$ \\
\hline $64-85-11-1752$ & 64 & $\underline{\mathrm{COI}}$ \\
\hline $64-85-11-1762$ & 64 & $\mathrm{CO1}$ \\
\hline $64-85-11-1763$ & 64 & $\overline{\mathrm{CO}}$ \\
\hline $64-85-11-1764$ & 64 & $\underline{\underline{\mathrm{CO1}}}$ \\
\hline $64-85-11-1765$ & 64 & $\mathrm{CO}$ \\
\hline $64-85-11-1784$ & 64 & $\mathrm{CO1}$ \\
\hline $64-85-11-1785$ & 64 & $\mathrm{COI}$ \\
\hline $64-85-11-1790$ & $\underline{\underline{64}}$ & $\underline{\mathrm{CO1}}$ \\
\hline $64-85-12-1912$ & $\underline{64}$ & $\underline{\mathrm{CO} 1}$ \\
\hline $64-86-02-154$ & 64 & $\overline{\mathrm{CO} 01}$ \\
\hline $64-86-02-155$ & 64 & $\mathrm{CO1}$ \\
\hline $64-86-03-233$ & 64 & $\mathrm{CO1}$ \\
\hline $64-86-03-234$ & $\underline{64}$ & $\overline{\mathrm{CO}}$ \\
\hline $64-86-03-203$ & 64 & $\mathrm{CO1}$ \\
\hline $64-86-03-207$ & 64 & $\mathrm{CO1}$ \\
\hline $64-86-03-231$ & $\underline{\underline{64}}$ & $\mathrm{COI}$ \\
\hline
\end{tabular}




\begin{tabular}{|l|l|l|}
\hline $\begin{array}{l}\text { PFP THERMAL } \\
\text { STABILIZATION }\end{array}$ & BLEND PLAN & $\begin{array}{l}\text { HNF-4989, REV. 1 } \\
\text { PAGE 22 OF 51 }\end{array}$ \\
\hline
\end{tabular}

\begin{tabular}{|c|c|c|}
\hline \multicolumn{3}{|c|}{ VAULT 1} \\
\hline$\overline{\text { ITEMID }}$ & CAT & COEI \\
\hline $64-86-03-235$ & 64 & $\mathrm{CO1}$ \\
\hline $64-86-03-236$ & 64 & $\mathrm{COI}$ \\
\hline $64-86-04-298$ & 64 & $\mathrm{CO1}$ \\
\hline $64-86-04-311$ & 64 & $\mathrm{CO1}$ \\
\hline $64-86-05-378$ & 64 & $\mathrm{CO1}$ \\
\hline $64-86-05-452$ & 64 & $\mathrm{CO1}$ \\
\hline $64-86-05-495$ & $\underline{\underline{64}}$ & $\mathrm{CO1}$ \\
\hline $64-86-07-580$ & 64 & $\mathrm{COI}$ \\
\hline $64-86-07-631$ & 64 & $\mathrm{CO1}$ \\
\hline $64-86-07-632$ & 64 & $\mathrm{CO1}$ \\
\hline $64-86-07-633$ & $\underline{64}$ & $\mathrm{CO1}$ \\
\hline $64-86-08-729$ & 64 & $\mathrm{CO1}$ \\
\hline $64-86-08-740$ & 64 & $\mathrm{CO1}$ \\
\hline $64-87-04-217$ & 64 & $\mathrm{CO1}$ \\
\hline $64-87-04-226$ & 64 & $\mathrm{CO1}$ \\
\hline $64-87-05-248$ & 64 & $\mathrm{CO1}$ \\
\hline $64-87-06-252$ & 64 & $\mathrm{CO1}$ \\
\hline $64-87-09-293$ & 64 & $\mathrm{CO1}$ \\
\hline $64-87-09-294$ & 64 & $\mathrm{CO1}$ \\
\hline $64-87-09-295$ & 64 & $\mathrm{CO1}$ \\
\hline $64-87-11-336$ & 64 & $\mathrm{CO1}$ \\
\hline $64-88-01-14$ & 64 & $\mathrm{CO}$ \\
\hline $64-88-01-16$ & 64 & $\mathrm{COI}$ \\
\hline $64-88-01-20$ & 64 & $\mathrm{CO1}$ \\
\hline $64-88-01-21$ & 64 & $\mathrm{COI}$ \\
\hline $64-88-02-28-1$ & 64 & $\mathrm{CO}$ \\
\hline $64-88-02-28-2$ & 64 & $\mathrm{CO1}$ \\
\hline $64-88-02-29$ & 6 & $\mathrm{CO1}$ \\
\hline $64-88-03-62-1$ & 64 & $\mathrm{COI}$ \\
\hline $64-88-03-62-2$ & 64 & $\overline{\mathrm{CO}}$ \\
\hline $64-88-05-89$ & 64 & $\mathrm{CO1}$ \\
\hline
\end{tabular}




\begin{tabular}{|l|l|l||}
\hline PFP THERMAL & BLEND PLAN & $\begin{array}{l}\text { HNF-4989, REV. 1 } \\
\text { PAGE 23 OF 51 }\end{array}$ \\
\hline
\end{tabular}

\begin{tabular}{|c|c|c|}
\hline \multicolumn{3}{|c|}{ VAULT 1} \\
\hline ITEMID & CAT & COEI \\
\hline $64-88-08-181$ & 64 & $\mathrm{COH}$ \\
\hline $64-88-08-191$ & 64 & $\mathrm{CO1}$ \\
\hline $63-88-11-327$ & 64 & $\mathrm{CO1}$ \\
\hline $63-88-11-330$ & 64 & $\mathrm{CO1}$ \\
\hline $67-77-03-308$ & 67 & $\mathrm{COI}$ \\
\hline $67-77-03-309$ & 67 & $\mathrm{CO1}$ \\
\hline $67-77-03-310$ & 67 & $\mathrm{COI}$ \\
\hline $67-77-03-311$ & 67 & $\mathrm{CO1}$ \\
\hline $67-77-03-312$ & 67 & $\mathrm{CO1}$ \\
\hline $67-77-03-313$ & 67 & $\mathrm{COI}$ \\
\hline $67-77-03-314$ & 67 & $\mathrm{CO1}$ \\
\hline $67-77-03-316$ & 67 & $\mathrm{CO}$ \\
\hline $67-77-03-317$ & 67 & $\mathrm{COI}$ \\
\hline $67-77-03-318$ & 67 & $\mathrm{CO1}$ \\
\hline $67-77-03-319$ & 67 & $\mathrm{CO1}$ \\
\hline $67-77-03-320$ & 67 & $\mathrm{CO1}$ \\
\hline $74-88-12-367$ & 74 & $\mathrm{COI}$ \\
\hline $16-x-5-6$ & 850 & 771 \\
\hline $83-X-2-16$ & 850 & 771 \\
\hline $204-x-284-12$ & 850 & 771 \\
\hline STD-208-X-100-6 & 850 & 771 \\
\hline $209-X-249-6$ & 850 & 771 \\
\hline $214-X-25-8$ & 850 & 771 \\
\hline $215-X-50-8$ & 850 & 771 \\
\hline $217-X-247-8$ & 850 & 771 \\
\hline $222-x-025-10$ & 850 & 771 \\
\hline STD $-225-X-243-10$ & 850 & 771 \\
\hline STD-259-X-168-24 & 850 & 771 \\
\hline $230-x-23-12$ & 850 & 771 \\
\hline $232-X-101-12$ & 850 & 771 \\
\hline $261-x-221-12$ & 850 & 771 \\
\hline
\end{tabular}




\begin{tabular}{|l|l|l|}
\hline $\begin{array}{l}\text { PFP THERMAL } \\
\text { STABILIZATION }\end{array}$ & BLEND PLAN & $\begin{array}{l}\text { HNF-4989, REV. 1 } \\
\text { PAGE 24 OF 51 }\end{array}$ \\
\hline
\end{tabular}

\begin{tabular}{|c|c|c|}
\hline \multicolumn{3}{|c|}{ VAULT 1} \\
\hline ITEMID & CAT & COEI \\
\hline GE-QC-10 & 850 & 771 \\
\hline GE-QC-14 & 850 & 771 \\
\hline$\overline{\mathrm{GE}-\mathrm{QC}-17}$ & 850 & $\underline{7711}$ \\
\hline$\overline{G E-Q C-18}$ & 850 & $\overline{771}$ \\
\hline$\overline{G E}-Q C-20$ & 850 & 771 \\
\hline$\overline{G E-Q C-21}$ & 850 & 771 \\
\hline$\overline{G E-Q C-22}$ & $\underline{850}$ & 771 \\
\hline GE-QC-23 & 850 & 771 \\
\hline$\overline{\mathrm{GE}-Q \mathrm{QC}-24}$ & 850 & 771 \\
\hline GE-QC-25 & 850 & 771 \\
\hline$\overline{G E-Q C-26}$ & 850 & 771 \\
\hline PPSL-183 & 51 & $\mathrm{CO2}$ \\
\hline$\overline{Q C-14}$ & 850 & 771 \\
\hline$\overline{Q C-15}$ & 850 & 771 \\
\hline QC-96-09-001 & 850 & 771 \\
\hline TD-1B & 51 & $\overline{\mathrm{CO} 01}$ \\
\hline TD-2 & 51 & $\mathrm{CO1}$ \\
\hline XDT-148-81-12 & 148 & 455 \\
\hline YEB-474-77-01-04 & 474 & $\mathrm{CO1}$ \\
\hline YEB-474-77-01-05 & 4774 & $\overline{C O 1}$ \\
\hline YEC-36-01-023 & 175 & 454 \\
\hline YDZ-36-10-01 & 175 & 455 \\
\hline$Y \mathrm{YDZ}-36-10-02$ & 175 & 455 \\
\hline YUD - 148-81-07 & 148 & $\underline{455}$ \\
\hline YUD-148-81-08-01 & 148 & 455 \\
\hline YUD-148-81-09 & 148 & 455 \\
\hline YUD-148-81-10 & 148 & 455 \\
\hline BLO-38-11-004 & 145 & 771 \\
\hline BLO-38-11-010 & 145 & 771 \\
\hline BLO-39-10-17-007 & $\overline{114}$ & 454 \\
\hline BLO-39-10-17-008 & $\overline{1114}$ & 454 \\
\hline
\end{tabular}




\begin{tabular}{|l|l|l||}
\hline $\begin{array}{l}\text { PFP THERMAL } \\
\text { STABILIZATION }\end{array}$ & BLEND PLAN & $\begin{array}{l}\text { HNF-4989, REV. 1 } \\
\text { PAGE 25 OF 51 }\end{array}$ \\
\hline
\end{tabular}

\begin{tabular}{|l|c|c|}
\hline \multicolumn{1}{|c|}{ ITEMID } & VAULT 1 & COEI \\
\hline \hline BLO-39-10-17-009 & 114 & 454 \\
\hline \hline BLO-39-10-17-010 & 114 & 454 \\
\hline \hline BLO-39-10-17-011 & 114 & 454 \\
\hline \hline BLO-39-10-17-012 & 114 & 454 \\
\hline \hline BLO-39-10-17-013 & 114 & 454 \\
\hline \hline BLO-39-11-05-004 & 114 & 454 \\
\hline \hline DZO-35-10-043-A & 175 & 454 \\
\hline \hline LAO-C10-234 & 112 & 454 \\
\hline \hline LAO-C10-242 & 112 & 454 \\
\hline \hline LAO-C10-245 & 112 & 454 \\
\hline \hline LAO-C10-247 & 112 & 454 \\
\hline \hline LAO-C10-259 & 112 & 454 \\
\hline \hline PBO-47-09-011-015 & 173 & 454 \\
\hline \hline PBO-47-09-011-028 & 173 & 454 \\
\hline \hline PBO-47-09-012-024 & 173 & 454 \\
\hline \hline PBO-47-09-012-025 & 173 & 454 \\
\hline \hline PBO-47-09-012-026 & 173 & 454 \\
\hline \hline PBO-47-09-013-004 & 173 & 454 \\
\hline \hline PBO-47-09-013-006 & 173 & 454 \\
\hline \hline PBO-47-09-013-007 & 173 \\
\hline \hline PBO-47-09-013-008 & 173 & \\
\hline
\end{tabular}

\begin{tabular}{|c|c|c|}
\hline \multicolumn{3}{|c|}{ VAULT 2} \\
\hline ITEMID & CAT & COEI \\
\hline$A-28-1-37$ & 111 & 746 \\
\hline AM-3H-01 SWEEP & 849 & $\mathrm{COI}$ \\
\hline AO-31-28 SWEEP & 849 & $\mathrm{CO1}$ \\
\hline ARF $-102-86-600$ & 102 & $\mathrm{CO1}$ \\
\hline AS-31H-57 SWEEP & 849 & $\mathrm{COI}$ \\
\hline $\mathrm{CAF}-482-75-47-2$ & 482 & C30 \\
\hline CE-5-203-8 & 74 & $\mathrm{C} 30$ \\
\hline$\overline{C E}-6-265-8$ & $\overline{74}$ & GOO \\
\hline
\end{tabular}




\begin{tabular}{|l|c|l|}
\hline \hline $\begin{array}{l}\text { PFP THERMAL } \\
\text { STABILIZATION }\end{array}$ & BLEND PLAN & $\begin{array}{l}\text { HNF-4989, REV. 1 } \\
\text { PAGE 26 OF 51 }\end{array}$ \\
\hline
\end{tabular}

\begin{tabular}{|c|c|c|}
\hline \multicolumn{3}{|c|}{ VAULT 2} \\
\hline ITEMID & CAT & $\overline{C O E I}$ \\
\hline$C Z A-465-72-08$ & 465 & $\mathrm{C01}$ \\
\hline$C Z A-465-72-23$ & 465 & $\mathrm{CO1}$ \\
\hline CZA-465-72-24 & 465 & $\overline{\mathrm{C} 01}$ \\
\hline CZA-465-72-25 & 465 & $\mathrm{CO1}$ \\
\hline CZA-469-74-228 & 455 & $\overline{\mathrm{CO} 1}$ \\
\hline EAB-3-2-1 $\quad$ PART & 67 & $\mathrm{CO1}$ \\
\hline EF-1-S SWEEPS & 849 & $\underline{\underline{\mathrm{C} 01}}$ \\
\hline GEO-30-08-001 & $\underline{20}$ & $\mathrm{CO1}$ \\
\hline GE-QC-27 & 850 & 771 \\
\hline HUA-40-10-01 & 148 & 454 \\
\hline HUA $-40-10-02$ & 148 & $\underline{\underline{454}}$ \\
\hline HUA- $40-10-03$ & 148 & 454 \\
\hline HUA-40-10-04 & 148 & 4454 \\
\hline S-22S-2 & 52 & $\overline{\mathrm{CO}}$ \\
\hline$\overline{Z \text { ZLS }-588-75-81}$ & $\underline{\underline{850}}$ & 771 \\
\hline $102-F-200-6$ & 850 & 771 \\
\hline $110-X S-38-6$ & 850 & 771 \\
\hline $104-X S-5-6$ & 850 & 771 \\
\hline $108-X S-25-6$ & 850 & 771 \\
\hline $145-174-X-7-6$ & 850 & $\underline{771}$ \\
\hline $19 \mathrm{~B} 14-\mathrm{A}$ & 175 & $\mathrm{CO1}$ \\
\hline $20-74-07-495$ & 20 & $\mathrm{COI}$ \\
\hline STD-207-X-50-6 & 850 & $\underline{\underline{7711}}$ \\
\hline $216-X-100-8$ & 850 & $\underline{\underline{771}}$ \\
\hline $223-x-50-10$ & 850 & $\underline{\underline{771}}$ \\
\hline $224-x-100-10$ & 850 & 771 \\
\hline $231-\mathrm{X}-51-12$ & 850 & $\underline{\underline{771}}$ \\
\hline $240-\mathrm{N}-1-12$ & 850 & $\underline{\underline{771}}$ \\
\hline $241-\mathrm{N}-2-12$ & 850 & 771 \\
\hline $242-\mathrm{N}-1-12$ & 850 & 771 \\
\hline $243-\mathrm{N}-5-12$ & 850 & 771 \\
\hline
\end{tabular}




\begin{tabular}{|l|l|l|}
\hline $\begin{array}{l}\text { PFP THERMAL } \\
\text { STABILIZATION }\end{array}$ & BLEND PLAN & $\begin{array}{l}\text { HNF-4989, REV. 1 } \\
\text { PAGE 27 OF 51 }\end{array}$ \\
\hline
\end{tabular}

\begin{tabular}{|c|c|c|}
\hline \multicolumn{3}{|c|}{ VAULT 2} \\
\hline ITEMID & $\overline{\mathrm{CAT}}$ & COEI \\
\hline $244-N-7-12$ & 850 & 771 \\
\hline $245-N-10-12$ & 850 & 771 \\
\hline $251 M-200-2$ & 850 & 771 \\
\hline $253 M-212-8$ & 850 & 771 \\
\hline $254 M-191-12$ & 850 & 771 \\
\hline $256 \mathrm{M}-161-22$ & 850 & 771 \\
\hline $263-N-2-8$ & 850 & 771 \\
\hline $265-N-5-8$ & 850 & 771 \\
\hline $266-N-7-8$ & 850 & 771 \\
\hline $39-X A-100-6$ & 850 & 771 \\
\hline $40-X A-150-6$ & 850 & 771 \\
\hline $41-85-08-1381$ & 41 & $\mathrm{CO} 1$ \\
\hline $42-86-07-625$ & 42 & $\mathrm{CO1}$ \\
\hline $42-86-08-707$ & 42 & $\mathrm{CO1}$ \\
\hline $42-86-08-743$ & 42 & $\mathrm{COI}$ \\
\hline $451-83-09-841$ & 451 & 781 \\
\hline $5-85-11-1726$ & 5 & $\mathrm{CO1}$ \\
\hline $60-184-1$ & 73 & EO4 \\
\hline $64-86-08-725$ & 64 & $\mathrm{COI}$ \\
\hline $64-86-08-727$ & 64 & $\mathrm{COI}$ \\
\hline $64-86-09-760$ & 64 & $\mathrm{CO1}$ \\
\hline $64-86-09-775$ & 64 & $\mathrm{CO1}$ \\
\hline $64-86-09-776$ & 64 & $\mathrm{CO1}$ \\
\hline $64-86-09-789$ & 64 & $\mathrm{CO1}$ \\
\hline $64-86-09-791$ & 64 & $\mathrm{CO1}$ \\
\hline $64-86-09-792$ & 64 & $\mathrm{CO1}$ \\
\hline $67-74-07-485$ & 67 & $\mathrm{CO3}$ \\
\hline $84-x-2-16$ & 850 & 771 \\
\hline $85-x-2-16$ & 850 & 771 \\
\hline $86-x-2-16$ & 850 & 771 \\
\hline $87-x-2-16$ & 850 & 771 \\
\hline
\end{tabular}




\begin{tabular}{|l|l|l||}
\hline $\begin{array}{l}\text { PFP THERMAL } \\
\text { STABILIZATION }\end{array}$ & BLEND PLAN & $\begin{array}{l}\text { HNF-4989, REV. 1 } \\
\text { PAGE 28 OF 51 }\end{array}$ \\
\hline
\end{tabular}

\begin{tabular}{|c|c|c|}
\hline \multicolumn{3}{|c|}{ VAULT 2} \\
\hline ITEMID & CAT & COEI \\
\hline $88-X-2-16$ & 850 & 771 \\
\hline $89-x-2-16$ & 850 & 771 \\
\hline $90-x-2-16$ & 850 & 771 \\
\hline $91-x-2-16$ & 850 & 771 \\
\hline $92-x-2-16$ & 850 & 771 \\
\hline $90-75-08-677$ & 91 & $\mathrm{CO1}$ \\
\hline $90-75-08-680$ & 91 & $\mathrm{CO1}$ \\
\hline $90-75-08-687$ & 91 & $\mathrm{CO1}$ \\
\hline$\angle A O-C 09-226$ & 112 & 4454 \\
\hline LAO-C10-226 & 112 & 4454 \\
\hline $\mathrm{LAO}-\mathrm{C} 11-226$ & 112 & 454 \\
\hline$\overline{\mathrm{LAO}-\mathrm{CO} 3-227}$ & 1112 & 454 \\
\hline$\overline{\mathrm{LAO}-\mathrm{CO} 4-227}$ & 112 & 454 \\
\hline $\mathrm{LAO}-\mathrm{CO} 5-227$ & 112 & 454 \\
\hline$\overline{\mathrm{LAO}-\mathrm{CO} 6-227}$ & 1112 & 454 \\
\hline $\mathrm{LAO}-\mathrm{C07}-227$ & 112 & 4454 \\
\hline $\mathrm{LAO}-\mathrm{C} 08-227$ & 112 & 2454 \\
\hline$\overline{\mathrm{LAO}-\mathrm{CO} 9-227}$ & 112 & 454 \\
\hline $\mathrm{LAO}-\mathrm{C} 10-227$ & 1112 & 454 \\
\hline$\overline{\mathrm{LAO}-\mathrm{C} 11-227}$ & 112 & 454 \\
\hline $\mathrm{LAO}-\mathrm{C} 12-227$ & 112 & 454 \\
\hline$\overline{\mathrm{LAO}-\mathrm{CO} 3-228}$ & 112 & 454 \\
\hline $\mathrm{IAO}-\mathrm{CO} 4-228$ & 112 & $\overline{4454}$ \\
\hline LAO - CO5-228 & $\overline{112}$ & 454 \\
\hline $\mathrm{LAO}-\mathrm{CO} 6-228$ & 112 & 2454 \\
\hline LAO-C07-228 & 1112 & 4454 \\
\hline$\overline{\mathrm{LAO}-\mathrm{CO} 8-228}$ & 112 & 4454 \\
\hline $\mathrm{LAO}-\mathrm{CO} 9-228$ & 112 & 2454 \\
\hline LAO-C11-228 & 112 & 454 \\
\hline $\mathrm{LAO}-\mathrm{C} 12-228$ & 112 & 4454 \\
\hline LAO-C13-228 & 112 & 454 \\
\hline
\end{tabular}




\begin{tabular}{|l|l|l|}
\hline $\begin{array}{l}\text { PFP THERMAL } \\
\text { STABILIZATION }\end{array}$ & BLEND PLAN & $\begin{array}{l}\text { HNF-4989, REV. 1 } \\
\text { PAGE 29 OF 51 }\end{array}$ \\
\hline
\end{tabular}

\begin{tabular}{|c|c|c|}
\hline \multicolumn{3}{|c|}{ VAUULT 2} \\
\hline ITEMID & $\overline{\mathrm{CAT}}$ & COEI \\
\hline LAO-C01-229 & 112 & 4454 \\
\hline$\overline{\mathrm{LAO}-\mathrm{C} 03-229}$ & 112 & 454 \\
\hline LAO-C04-229 & 112 & 454 \\
\hline LAO-C05-229 & 112 & 454 \\
\hline LAO-C06-229 & 112 & 454 \\
\hline LAO-C07-229 & 112 & 454 \\
\hline LAO-C08-229 & $\overline{112}$ & 454 \\
\hline $\mathrm{LAO}-\mathrm{CO} 2-230$ & 112 & 454 \\
\hline $\mathrm{LAO}-\mathrm{CO} 3-230$ & $\overline{112}$ & $\overline{454}$ \\
\hline LAO-C04-230 & $\overline{112}$ & $\overline{454}$ \\
\hline$\overline{\mathrm{LAO}-\mathrm{C} 05-230}$ & $\overline{112}$ & 454 \\
\hline LAO-C06-230 & $\overline{112}$ & 4454 \\
\hline LAO-C07-230 & 112 & 454 \\
\hline$\overline{\mathrm{LAO}-\mathrm{C} 08-230}$ & 112 & 454 \\
\hline$\overline{L A O}-\mathrm{CO} 9-230$ & $\overline{112}$ & 454 \\
\hline LAO-C02-231 & $\overline{112}$ & $\overline{454}$ \\
\hline $\mathrm{LAO}-\mathrm{C} 03-231$ & 112 & 454 \\
\hline$\overline{L A O}-\mathrm{C} 04-231$ & 112 & 454 \\
\hline$\overline{L A O}-\mathrm{C} 05-231$ & 112 & 454 \\
\hline$\overline{\mathrm{LAO}-\mathrm{C} 06-231}$ & $\overline{112}$ & 454 \\
\hline$\overline{\mathrm{LAO}-\mathrm{C} 07-231}$ & $\overline{112}$ & 454 \\
\hline$\overline{\mathrm{IAO}-\mathrm{CO} 8-231}$ & $\overline{112}$ & 454 \\
\hline LAO-C09-231 & 112 & 454 \\
\hline $\mathrm{LAO}-\mathrm{C} 11-231$ & 112 & 454 \\
\hline $\mathrm{LAO}-\mathrm{C} 12-231$ & 112 & 454 \\
\hline $\mathrm{LAO}-\mathrm{C} 13-231$ & 112 & 454 \\
\hline$\overline{\mathrm{L}} \mathrm{AO}-\mathrm{CO} 2-232$ & $\overline{112}$ & 454 \\
\hline LAO-C03-232 & $\overline{112}$ & $\overline{454}$ \\
\hline$\overline{\mathrm{L} A \mathrm{AO}-\mathrm{C} 04-232}$ & 112 & $\overline{454}$ \\
\hline$\overline{\mathrm{LAO}-\mathrm{C} 06-232}$ & 112 & 454 \\
\hline$\overline{\mathrm{LAO}-\mathrm{C} 07-232}$ & 112 & 454 \\
\hline
\end{tabular}




\begin{tabular}{|l|l|l|}
\hline $\begin{array}{l}\text { PFP THERMAL } \\
\text { STABILIZATION }\end{array}$ & BLEND PLAN & $\begin{array}{l}\text { HNF-4989, REV. 1 } \\
\text { PAGE 30 OF 51 }\end{array}$ \\
\hline
\end{tabular}

\begin{tabular}{|l|c|c|}
\hline \multicolumn{1}{|c|}{ VAULT 2 } \\
\hline \hline ITEMID & CAT & COEI \\
\hline \hline LAO-C02-233 & 112 & 454 \\
\hline \hline LAO-C03-233 & 112 & 454 \\
\hline \hline LAO-CO4-233 & 112 & 454 \\
\hline \hline LAO-C05-233 & 112 & 454 \\
\hline \hline LAO-C06-233 & 112 & 454 \\
\hline
\end{tabular}

\begin{tabular}{|c|c|c|}
\hline \multicolumn{3}{|c|}{ VAULT 4} \\
\hline ITEMID & CAT & COEI \\
\hline $10-89-07-84$ & 10 & $\mathrm{COI}$ \\
\hline $10-89-07-88$ & 10 & $\mathrm{COI}$ \\
\hline 22260 & 112 & 454 \\
\hline 22277 & 112 & 454 \\
\hline $41 \mathrm{~A}$ & 51 & $\mathrm{COO}$ \\
\hline $41 B$ & 51 & $\mathrm{COO}$ \\
\hline $41-89-05-36$ & 41 & G05 \\
\hline $41-89-05-37$ & 41 & G05 \\
\hline $41-89-05-38$ & 41 & G05 \\
\hline $41-89-05-39$ & $4 \underline{41}$ & G05 \\
\hline $41-89-07-44$ & 41 & G05 \\
\hline $41-89-07-45$ & 41 & G05 \\
\hline $42-87-02-26$ & 42 & G05 \\
\hline $61-85-04-580$ & 5 & $\mathrm{C01}$ \\
\hline $61-85-08-1222$ & 5 & $\mathrm{COI}$ \\
\hline $61-85-08-1223$ & 5 & $\mathrm{CO1}$ \\
\hline $6-85-01-46$ & 6 & $\mathrm{COI}$ \\
\hline $6-85-01-85$ & $\overline{6}$ & $\mathrm{CO1}$ \\
\hline $6-85-02-131$ & $\underline{\underline{6}}$ & $\mathrm{CO1}$ \\
\hline $61-84-10-335$ & 61 & $\mathrm{CO1}$ \\
\hline $61-84-10-339$ & 61 & $\mathrm{CO1}$ \\
\hline $61-84-10-389$ & 61 & $\mathrm{CO1}$ \\
\hline $61-85-03-331$ & 61 & $\overline{C O I}$ \\
\hline $61-85-03-336$ & 61 & $\mathrm{CO1}$ \\
\hline
\end{tabular}




\begin{tabular}{|l|l|l|}
\hline $\begin{array}{l}\text { PFP THERMAL } \\
\text { STABILIZATION }\end{array}$ & BLEND PLAN & $\begin{array}{l}\text { HNF-4989, REV. 1 } \\
\text { PAGE 31 OF 51 }\end{array}$ \\
\hline
\end{tabular}

\begin{tabular}{|c|c|c|}
\hline \multicolumn{3}{|c|}{ VAULT 4} \\
\hline ITEMID & $\overline{C \overline{C A T}}$ & COEI \\
\hline $61-85-03-337$ & 61 & $\mathrm{CO1}$ \\
\hline $61-85-03-339$ & 61 & $\overline{\mathrm{CO} 01}$ \\
\hline $61-85-03-482$ & 61. & $\mathrm{CO1}$ \\
\hline $61-85-03-484$ & 61 & $\mathrm{CO} 1$ \\
\hline $61-85-03-533$ & 61 & $\mathrm{CO}$ \\
\hline $61-85-03-543$ & 61 & $\mathrm{CO}$ \\
\hline $61-85-04-581$ & $\underline{61}$ & $\overline{\mathrm{COI}}$ \\
\hline $61-85-04-613$ & 61 & $\overline{\mathrm{CO1}}$ \\
\hline $61-85-04-614$ & 61 & $\mathrm{CO} 1$ \\
\hline $61-85-04-622$ & 61 & $\mathrm{CO} 1$ \\
\hline $61-85-05-643$ & 61 & $\mathrm{CO1}$ \\
\hline $61-85-05-644$ & 61 & $\mathrm{C01}$ \\
\hline $61-85-05-645$ & 61 & $\mathrm{CO1}$ \\
\hline $61-85-05-651$ & 61 & $\mathrm{COI}$ \\
\hline $61-85-05-652$ & 61 & $\mathrm{CO1}$ \\
\hline $61-85-05-820$ & $\underline{61}$ & $\mathrm{CO1}$ \\
\hline $61-85-06-953$ & 61 & $\mathrm{CO1}$ \\
\hline $61-85-07-1018-2$ & 61 & $\mathrm{CO1}$ \\
\hline $61-85-07-1019$ & $\overline{61}$ & $\overline{\mathrm{CO} 01}$ \\
\hline $61-85-07-1020$ & 61 & $\mathrm{CO1}$ \\
\hline $61-85-07-1028$ & 61 & $\mathrm{CO1}$ \\
\hline $61-85-07-1029$ & 61 & $\mathrm{COI}$ \\
\hline $61-85-07-1030$ & 61 & $\mathrm{CO1}$ \\
\hline $61-85-07-1031$ & 61 & $\mathrm{CO1}$ \\
\hline $61-85-07-1053$ & 61 & $\overline{\mathrm{CO}}$ \\
\hline $61-85-07-1054$ & 61 & $\mathrm{COI}$ \\
\hline $61-85-07-1055$ & $\underline{\underline{61}}$ & $\mathrm{CO1}$ \\
\hline $61-85-07-1056$ & $\underline{61}$ & $\underline{\mathrm{C} 01}$ \\
\hline $61-85-07-1057$ & 61 & $\mathrm{CO1}$ \\
\hline $61-85-07-1058$ & 61 & $\mathrm{CO1}$ \\
\hline $61-85-07-1059$ & 61 & $\mathrm{COI}$ \\
\hline
\end{tabular}




\begin{tabular}{|l|l|l||}
\hline $\begin{array}{l}\text { PFP THERMAL } \\
\text { STABILIZATION }\end{array}$ & BLEND PLAN & $\begin{array}{l}\text { HNF-4989, REV. 1 } \\
\text { PAGE 32 OF 51 }\end{array}$ \\
\hline
\end{tabular}

\begin{tabular}{|c|c|c|}
\hline \multicolumn{3}{|c|}{ VAULT 4} \\
\hline ITEMID & $\mathrm{CAT}$ & COEI \\
\hline $61-85-07-1060$ & 61 & $\overline{C O 1}$ \\
\hline $61-85-07-1061$ & 61. & G00 \\
\hline $61-85-07-1137$ & 61 & $\underline{\underline{\mathrm{CO} 1}}$ \\
\hline $61-85-07-1138$ & 61 & $\underline{\mathrm{CO1}}$ \\
\hline $61-85-07-1139$ & 61 & $\mathrm{CO1}$ \\
\hline $61-85-07-1140$ & 61 & $\mathrm{CO1}$ \\
\hline $61-85-07-1141$ & $\underline{61}$ & $\overline{\mathrm{COI}}$ \\
\hline $61-85-07-1142$ & $\underline{\underline{61}}$ & $\mathrm{CO1}$ \\
\hline $61-85-07-1143$ & 61 & $\mathrm{CO1}$ \\
\hline $61-85-07-1144$ & 61 & $\overline{\mathrm{CO}}$ \\
\hline $61-85-07-1145$ & $\underline{61}$ & $\underline{\mathrm{CO1}}$ \\
\hline $61-85-07-1146$ & 61 & $\underline{\mathrm{CO1}}$ \\
\hline $61-85-07-1147$ & 61 & $\mathrm{CO1}$ \\
\hline $61-85-07-1148$ & 61 & $\overline{\mathrm{CO1}}$ \\
\hline $61-85-07-2000$ & 61 & $\overline{\mathrm{CO}}$ \\
\hline $61-85-07-2001$ & 61 & $\overline{\mathrm{CO}}$ \\
\hline $61-85-07-2002$ & $\underline{61}$ & $\mathrm{CO1}$ \\
\hline $61-85-07-2003$ & 61 & $\mathrm{CO1}$ \\
\hline $61-85-08-1189$ & $\underline{\underline{61}}$ & $\mathrm{CO1}$ \\
\hline $61-85-08-1190$ & 61 & C01 \\
\hline $61-85-08-1191$ & 61 & $\mathrm{CO1}$ \\
\hline $61-85-08-1192$ & 67 & $\overline{\mathrm{COI}}$ \\
\hline $61-85-08-1205$ & 61 & $\overline{\mathrm{COI}}$ \\
\hline $61-85-08-1206$ & 61 & $\overline{\mathrm{CO}}$ \\
\hline $61-85-08-1207$ & $\overline{61}$ & $\mathrm{CO1}$ \\
\hline $61-85-08-1224$ & 61 & $\mathrm{CO1}$ \\
\hline $61-85-08-1232$ & $\underline{\underline{61}}$ & $\underline{\underline{\mathrm{CO1}}}$ \\
\hline $61-85-08-1233$ & $\underline{61}$ & $\mathrm{C01}$ \\
\hline $61-85-08-1234$ & $\overline{61}$ & $\overline{\mathrm{COI}}$ \\
\hline $61-85-08-1235$ & 61 & $\mathrm{CO1}$ \\
\hline $61-85-08-1236$ & 61 & $\mathrm{CO1}$ \\
\hline
\end{tabular}




\begin{tabular}{|l|l|l|}
\hline $\begin{array}{l}\text { PFP THERMAL } \\
\text { STABILIZATION }\end{array}$ & BLEND PLAN & $\begin{array}{l}\text { HNF-4989, REV. 1 } \\
\text { PAGE 33 OF 51 }\end{array}$ \\
\hline
\end{tabular}

\begin{tabular}{|c|c|c|}
\hline \multicolumn{3}{|c|}{ VĀULT 4} \\
\hline ITEMID & CAT & COEI \\
\hline $61-85-08-1237$ & 61 & $\mathrm{COI}$ \\
\hline $61-85-08-1238$ & 61 & $\mathrm{CO1}$ \\
\hline $61-85-08-1245$ & 61 & $\mathrm{CO1}$ \\
\hline $61-85-08-1246$ & 61 & $\mathrm{COI}$ \\
\hline $61-85-08-1247$ & 61 & $\mathrm{COI}$ \\
\hline $61-85-08-1248$ & 61 & $\mathrm{COI}$ \\
\hline $61-85-08-1264$ & 61 & $\mathrm{CO1}$ \\
\hline $61-85-08-1265$ & 61 & $\mathrm{CO1}$ \\
\hline $61-85-08-1267$ & 61 & $\mathrm{CO1}$ \\
\hline $61-85-08-1276$ & 61 & $\mathrm{CO1}$ \\
\hline $61-85-08-1277$ & $\underline{\underline{61}}$ & $\underline{\mathrm{CO1}}$ \\
\hline $61-85-08-1278$ & 61 & $\mathrm{CO1}$ \\
\hline $61-85-08-1296$ & 61 & $\mathrm{COH}$ \\
\hline $61-85-08-1297$ & 61 & $\mathrm{CO1}$ \\
\hline $61-85-08-1318$ & 61 & $\mathrm{COI}$ \\
\hline $61-85-08-1319$ & 61 & CO1 \\
\hline $61-85-08-1320$ & 61 & $\mathrm{COI}$ \\
\hline $61-85-08-1321$ & 61 & $\mathrm{CO1}$ \\
\hline $61-85-08-1329$ & 61 & $\mathrm{CO1}$ \\
\hline $61-85-08-1330$ & 61 & $\mathrm{CO1}$ \\
\hline $61-85-08-1331$ & 61 & $\mathrm{CO1}$ \\
\hline $61-85-08-1333$ & 61 & $\mathrm{CO1}$ \\
\hline $61-85-08-1342$ & 61 & $\mathrm{COI}$ \\
\hline $61-85-08-1343$ & 61 & $\mathrm{CO1}$ \\
\hline $61-85-08-1345$ & 61 & $\mathrm{COH}$ \\
\hline $61-85-08-1358$ & 61 & $\mathrm{CO}$ \\
\hline $61-85-08-1359$ & 61 & $\mathrm{CO1}$ \\
\hline $61-85-08-1361$ & 61 & $\mathrm{CO1}$ \\
\hline $61-85-08-1367$ & 61 & $\mathrm{CO1}$ \\
\hline $61-85-08-1368$ & 61 & $\mathrm{CO1}$ \\
\hline $61-85-08-1369$ & 61 & $\mathrm{COI}$ \\
\hline
\end{tabular}




\begin{tabular}{|l|l|l|}
\hline $\begin{array}{l}\text { PFP THERMAL } \\
\text { STABILIZATION }\end{array}$ & BLEND PLAN & $\begin{array}{l}\text { HNF-4989, REV. 1 } \\
\text { PAGE 34 OF 51 }\end{array}$ \\
\hline
\end{tabular}

\begin{tabular}{|c|c|c|}
\hline \multicolumn{3}{|c|}{ VĀULT 4} \\
\hline ITEMID & CAT & COEI \\
\hline $61-85-08-1370$ & 61 & $\mathrm{CO} 1$ \\
\hline $61-85-08-1371$ & 61 & $\mathrm{CO1}$ \\
\hline $62-83-06-363$ & 62 & $\mathrm{COO}$ \\
\hline $62-85-06-960$ & 62 & $\mathrm{COI}$ \\
\hline $62-85-09-1384$ & 62 & $\mathrm{CO} 1$ \\
\hline $62-85-09-1386$ & 62 & $\mathrm{CO1}$ \\
\hline $62-85-09-1393$ & 62 & $\mathrm{CO1}$ \\
\hline $62-85-11-1795$ & 62 & $\mathrm{CO1}$ \\
\hline $62-85-12-1829$ & 62 & $\mathrm{CO1}$ \\
\hline $62-86-03-205$ & 62 & $\mathrm{COI}$ \\
\hline $62-86-03-220$ & 62 & $\mathrm{COI}$ \\
\hline $62-87-03-64$ & 62 & $\mathrm{CO1}$ \\
\hline $62-87-10-325$ & 62 & $\mathrm{CO1}$ \\
\hline $61-85-03-332-2$ & 63 & $\mathrm{COI}$ \\
\hline $63-85-04-612$ & 63 & $\mathrm{CO1}$ \\
\hline $63-87-02-56$ & 63 & $\mathrm{CO1}$ \\
\hline $63-87-03-66$ & 63 & $\mathrm{CO}$ \\
\hline $63-87-03-67$ & 63 & $\mathrm{CO1}$ \\
\hline $63-87-03-92$ & 63 & $\mathrm{CO1}$ \\
\hline $63-87-08-278$ & 63 & $\mathrm{CO1}$ \\
\hline $63-87-08-283$ & 63 & $\mathrm{COI}$ \\
\hline $63-87-10-327$ & 63 & $\mathrm{CO}$ \\
\hline $64-86-12-1029$ & 63 & $\mathrm{CO}$ \\
\hline $64-85-10-1545$ & 64 & $\mathrm{CO1}$ \\
\hline $64-85-10-1561$ & 64 & C01 \\
\hline $64-85-10-1570$ & 64 & $\mathrm{CO1}$ \\
\hline $64-85-10-1579$ & 64 & $\mathrm{CO1}$ \\
\hline $64-85-10-1588$ & 64 & $\underline{\mathrm{CO1}}$ \\
\hline $64-85-12-1830$ & 64 & $\mathrm{COI}$ \\
\hline $64-85-12-1858$ & 64 & $\mathrm{CO1}$ \\
\hline $64-85-12-1859$ & 64 & $\mathrm{CO1}$ \\
\hline
\end{tabular}




\begin{tabular}{|l|l|l|}
\hline $\begin{array}{l}\text { PFP THERMAL } \\
\text { STABILIZATION }\end{array}$ & BLEND PLAN & $\begin{array}{l}\text { HNF-4989, REV. } 1 \\
\text { PAGE 35 OF 51 }\end{array}$ \\
\hline
\end{tabular}

\begin{tabular}{|c|c|c|}
\hline \multicolumn{3}{|c|}{ VAULT 4} \\
\hline ITEMID & $\overline{\mathrm{CATT}}$ & $\overline{\mathrm{COEI}}$ \\
\hline $64-85-12-1860-1$ & 64 & $\overline{\mathrm{CO}}$ \\
\hline $64-85-12-1860-2$ & 64 & $\mathrm{COI}$ \\
\hline $64-85-12-1861$ & 64 & $\mathrm{CO1}$ \\
\hline $64-85-12-1871$ & 64 & $\mathrm{CO1}$ \\
\hline $64-85-12-1872$ & 64 & $\mathrm{CO1}$ \\
\hline $64-85-12-1873$ & 64 & $\mathrm{CO} 1$ \\
\hline $64-85-12-1874$ & 64 & $\mathrm{CO1}$ \\
\hline $64-85-12-1884$ & 64 & CO1 \\
\hline $64-85-12-1885$ & 64 & $\mathrm{CO1}$ \\
\hline $64-86-03-206$ & 64 & $\mathrm{CO} 1$ \\
\hline $64-86-04-299$ & 64 & C01 \\
\hline $64-86-05-376$ & 64 & $\mathrm{CO1}$ \\
\hline $64-86-09-814$ & 64 & $\mathrm{CO} 1$ \\
\hline $64-86-09-817$ & 64 & $\mathrm{COI}$ \\
\hline $64-86-10-850$ & 64 & $\mathrm{COI}$ \\
\hline $64-86-10-851$ & 64 & $\mathrm{C01}$ \\
\hline $64-87-03-154$ & 64 & $\mathrm{CO}$ \\
\hline $64-95-02-08$ & 64 & $\mathrm{CO2}$ \\
\hline $64-95-02-21$ & $\underline{64}$ & $\mathrm{CO2}$ \\
\hline $64-95-06-153$ & $\underline{\underline{64}}$ & $\mathrm{CO2}$ \\
\hline $64-95-09-216$ & 64 & $\mathrm{CO2}$ \\
\hline $64-95-09-217$ & 64 & $\mathrm{CO2}$ \\
\hline $64-95-10-220$ & 64 & $\mathrm{CO2}$ \\
\hline $64-96-01-014$ & $\underline{64}$ & $\mathrm{CO2}$ \\
\hline $64-96-01-015$ & 64 & C02 \\
\hline $64-96-05-214$ & 64 & $\mathrm{CO2}$ \\
\hline $\mathrm{ACO}-29-12-001 \quad \mathrm{~S}$ & $\underline{145}$ & 771 \\
\hline 3707 & 51 & $\mathrm{COO}$ \\
\hline $\mathrm{CZA}-001$ & 41 & G05 \\
\hline $\mathrm{CZA}-001$ & 148 & 454 \\
\hline GE-QC-7 & 850 & 771 \\
\hline
\end{tabular}




\begin{tabular}{|l|l|l|}
\hline PFP THERMAL & BLEND PLAN & $\begin{array}{l}\text { HNF-4989, REV. 1 } \\
\text { PAGE 36 OF 51 }\end{array}$ \\
\hline
\end{tabular}

\begin{tabular}{|c|c|c|}
\hline \multicolumn{3}{|c|}{ VAULT 4} \\
\hline ITEMID & CAT & COEI \\
\hline GE-QC-8 & 850 & 771 \\
\hline GE-QC-9 & 850 & 771 \\
\hline GES - 33-06-001 & 175 & 454 \\
\hline $\mathrm{K} 412$ & $\underline{\underline{51}}$ & $\mathrm{COO}$ \\
\hline$N X-29-02-563-31$ & 145 & 771 \\
\hline $\mathrm{NX}-29-02-563-32$ & 145 & 771 \\
\hline $\mathrm{NX}-29-03-\mathrm{S} 56-27$ & 145 & 771 \\
\hline $\mathrm{NX}-29-03-556-28$ & 145 & 771 \\
\hline $\mathrm{NX}-29-03-556-29$ & 145 & 771 \\
\hline $0-30-03-4-5$ & 145 & 771 \\
\hline $0-30-09-001 \quad C$ & 145 & 771 \\
\hline WBO $-30-10-1-2$ & 145 & 771 \\
\hline ZPS-28-09-049-40-A & 145 & 771 \\
\hline ZPS-28-12-019-19 & 145 & 771 \\
\hline ZYP-30-09-001-0001 & 145 & 771 \\
\hline ZYP-30-09-001-0002 & 145 & 771 \\
\hline PPSL-102 & 51 & $\mathrm{COO}$ \\
\hline PPSL-103 & 51 & $\mathrm{COO}$ \\
\hline PPSL-104 & 51 & $\underline{\underline{\mathrm{COO}}}$ \\
\hline PPSL-225 & 51 & $\mathrm{CO1}$ \\
\hline BLO-39-10-021-002 & 114 & 454 \\
\hline BLO-39-10-021-003 & 114 & 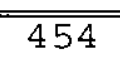 \\
\hline BLO-39-10-021-004 & $\overline{114}$ & 454 \\
\hline BLO-39-10-021-005 & $\overline{114}$ & 2454 \\
\hline BLO-39-10-021-006 & 114 & 454 \\
\hline BLO-39-10-021-007 & 114 & 454 \\
\hline BLO-39-10-021-008 & 114 & 454 \\
\hline BLO-39-10-021-009 & 114 & 454 \\
\hline BLO-39-10-021-010 & 114 & 454 \\
\hline BLO-39-10-021-011 & $\overline{114}$ & 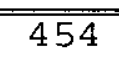 \\
\hline BLO-39-10-16-011 & 114 & 454 \\
\hline
\end{tabular}




\begin{tabular}{|l|l|l|}
\hline $\begin{array}{l}\text { PFP THERMAL } \\
\text { STABILIZATION }\end{array}$ & BLEND PLAN & $\begin{array}{l}\text { HNF-4989, REV. 1 } \\
\text { PAGE 37 OF 51 }\end{array}$ \\
\hline
\end{tabular}

\begin{tabular}{|c|c|c|}
\hline \multicolumn{3}{|c|}{ VAULT 4} \\
\hline ITEMID & CAT & COEI \\
\hline BLO-39-10-16-012 & 114 & 454 \\
\hline BLO-39-10-18-001 & 114 & 454 \\
\hline BLO-39-10-18-002 & 114 & 454 \\
\hline BLO-39-10-18-003 & 114 & 454 \\
\hline$\overline{\mathrm{BLO}-39-10} \overline{=18-004}$ & 114 & 454 \\
\hline BLO-39-10-18-005 & 114 & 454 \\
\hline BLO-39-10-18-006 & 114 & 454 \\
\hline BLO-39-10-18-007 & 114 & 4554 \\
\hline BLO-39-10-18-009 & 114 & 454 \\
\hline BLO-39-10-19-001 & 114 & 454 \\
\hline BLO-39-10-19-003 & 114 & 454 \\
\hline BLO-39-10-19-005 & 114 & 454 \\
\hline BLO-39-10-19-009 & 114 & 454 \\
\hline BLO-39-10-19-012 & 114 & 454 \\
\hline BLO-39-10-20-001 & 1114 & 454 \\
\hline BLO-39-10-20-003 & 114 & 454 \\
\hline BLO-39-10-20-005 & 114 & 454 \\
\hline BLO-39-10-20-006 & 114 & 454 \\
\hline BLO-39-10-20-007 & 114 & 454 \\
\hline BLO-39-10-20-010 & 114 & 454 \\
\hline BLO-39-10-20-011 & 114 & 454 \\
\hline BLO-39-10-20-012 & $\overline{114}$ & 454 \\
\hline BLO-39-11-01-003 & 114 & 454 \\
\hline BLO-39-11-01-004 & 114 & 454 \\
\hline BLO-39-11-01-005 & 114 & 454 \\
\hline BLO-39-11-01-007 & 114 & 454 \\
\hline BLO-39-11-01-008 & 114 & 454 \\
\hline BLO-39-11-01-012 & 1.14 & 454 \\
\hline BLO-39-11-01-013 & 1114 & 454 \\
\hline BLO-39-11-01-014 & 114 & 454 \\
\hline BLO-39-11-02-002 & 1114 & 454 \\
\hline
\end{tabular}




\begin{tabular}{|l|l|l|}
\hline $\begin{array}{l}\text { PFP THERMAL } \\
\text { STABILIZATION }\end{array}$ & BLEND PLAN & $\begin{array}{l}\text { HNF-4989, REV. 1 } \\
\text { PAGE 38 OF 51 }\end{array}$ \\
\hline
\end{tabular}

\begin{tabular}{|c|c|c|}
\hline \multicolumn{3}{|c|}{ VAULT 4} \\
\hline ITEMID & CAT & COEI \\
\hline BLO-39-11-02-003 & 114 & 454 \\
\hline BLO-39-11-02-005 & 114 & 454 \\
\hline BLO-39-11-02-007 & $\overline{114}$ & 454 \\
\hline BLO-39-11-02-009 & 114 & $4 \overline{454}$ \\
\hline BLO-39-11-02-010 & 114 & 454 \\
\hline BLO-39-11-02-01.1 & 114 & 454 \\
\hline BLO-39-11-02-012 & 1114 & 4554 \\
\hline BLO-39-11-02-013 & 1114 & 454 \\
\hline BLO-39-11-02-014 & 114 & 454 \\
\hline BLO-39-11-03-001 & 114 & $4 \overline{454}$ \\
\hline BLO-39-11-03-002 & $\overline{114}$ & $\overline{454}$ \\
\hline BLO-39-11-03-003 & 114 & 454 \\
\hline BLO-39-11-03-004 & 1114 & 4554 \\
\hline BLO-39-11-03-005 & 114 & 454 \\
\hline BLO-39-11-03-006 & 114 & $\overline{454}$ \\
\hline BLO-39-11-03-007 & 1114 & 454 \\
\hline BLO-39-11-03-008 & 114 & 4554 \\
\hline BLO-39-11-03-009 & 114 & 454 \\
\hline BLO-39-11-03-010 & 1114 & 454 \\
\hline BLO-39-11-03-011 & 114 & 454 \\
\hline BLO-39-11-03-012 & 114 & 454 \\
\hline BLO-39-11-03-013 & 114 & 454 \\
\hline BLO-39-11-03-014 & 114 & 454 \\
\hline BLO-39-11-04-001 & 114 & 454 \\
\hline BLO-39-11-04-002 & 114 & 454 \\
\hline $\mathrm{BLO}-39-11-04-003$ & 114 & 454 \\
\hline BLO-39-11-04-004 & 114 & 454 \\
\hline BLO-39-11-04-005 & 114 & 454 \\
\hline BLO-39-11-04-006 & 114 & 454 \\
\hline BLO-39-11-04-007 & 114 & 454 \\
\hline BLO-39-11-04-008 & 114 & 454 \\
\hline
\end{tabular}




\begin{tabular}{|l|l|l|}
\hline $\begin{array}{l}\text { PFP THERMAL } \\
\text { STABILIZATION }\end{array}$ & BLEND PLAN & $\begin{array}{l}\text { HNF-4989, REV. 1 } \\
\text { PAGE 39 OF 51 }\end{array}$ \\
\hline
\end{tabular}

\begin{tabular}{|c|c|c|}
\hline \multicolumn{3}{|c|}{ VAULT 4} \\
\hline ITEMID & $\mathrm{CAT}$ & $\overline{C O E I}$ \\
\hline BLO-39-11-04-009 & 114 & 454 \\
\hline BLO-39-11-04-010 & 1114 & $\overline{4454}$ \\
\hline BLO-39-11-04-011 & 1114 & "454 \\
\hline BLO-39-11-04-012 & 114 & 454 \\
\hline BLO-39-11-04-013 & 1114 & 454 \\
\hline BLO-39-11-04-014 & 114 & 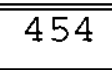 \\
\hline BLO-39-11-05-001 & 114 & (454 \\
\hline BLO-39-11-05-002 & 114 & "454 \\
\hline BLO-39-11-05-003 & 114 & 454 \\
\hline BLO-39-11-05-004 & 1114 & 454 \\
\hline BLO-39-11-05-005 & 114 & 454 \\
\hline BLO-39-11-05-006 & 114 & 454 \\
\hline BLO-39-11-05-007 & 114 & 454 \\
\hline BLO-39-11-05-008 & $\overline{114}$ & 454 \\
\hline BLO-39-11-05-009 & 114 & $\overline{c 454}$ \\
\hline BLO-39-11-05-010 & 114 & 454 \\
\hline BLO-39-11-05-011 & 114 & 454 \\
\hline $\mathrm{BLO}-39-11-05-012$ & 114 & 454 \\
\hline BLO-39-11-05-013 & 114 & 454 \\
\hline BLO-39-11-06-001 & 114 & 454 \\
\hline BLO-39-11-06-002 & 114 & 454 \\
\hline BLO-39-11-06-003 & 114 & 454 \\
\hline BLO-39-11-06-004 & 114 & 454 \\
\hline BLO-39-11-06-005 & 114 & 454 \\
\hline $\mathrm{BLO}-39-11-06-006$ & 1114 & 454 \\
\hline BLO-39-11-06-007 & 114 & 454 \\
\hline BLO-39-11-06-008 & 114 & 454 \\
\hline BLO-39-11-06-009 & 1114 & 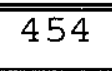 \\
\hline BLO-39-11-06-010 & 114 & $\overline{454}$ \\
\hline BLO-39-11-06-011 & 114 & 454 \\
\hline BLO-39-11-06-012 & 114 & 454 \\
\hline
\end{tabular}




\begin{tabular}{|l|l|l|}
\hline $\begin{array}{l}\text { PFP THERMAL } \\
\text { STABILIZATION }\end{array}$ & BLEND PLAN & $\begin{array}{l}\text { HNF-4989, REV. 1 } \\
\text { PAGE 40 OF 51 }\end{array}$ \\
\hline
\end{tabular}

\begin{tabular}{|c|c|c|}
\hline \multicolumn{3}{|c|}{ VAULT 4} \\
\hline ITEMID & CAT & COEI \\
\hline BLO-39-11-06-013 & $\overline{1114}$ & $\overline{454}$ \\
\hline BLO-39-11-06-014 & 114 & 454 \\
\hline BLO-39-11-07-001 & 114 & 4454 \\
\hline BLO-39-11-07-002 & 1114 & 4454 \\
\hline BLO-39-11-07-003 & 1114 & $\overline{4454}$ \\
\hline BLO-39-11-07-004 & 114 & 454 \\
\hline BLO-39-11-07-005 & 114 & 454 \\
\hline BLO-39-11-07-006 & $\overline{114}$ & $\overline{4454}$ \\
\hline BLO-39-11-07-007 & 114 & 4454 \\
\hline BLO-39-11-07-008 & 114 & 454 \\
\hline BLO-39-11-07-009 & 114 & 454 \\
\hline$\overline{\mathrm{BLO}-39-11-07-010}$ & 114 & 454 \\
\hline BLO-39-11-08-006 & 114 & 454 \\
\hline$\overline{\mathrm{BLO}-39-11-08-009}$ & 1114 & 454 \\
\hline BLO-39-11-08-010 & 114 & 454 \\
\hline BLO-39-11-08-011 & 1114 & 454 \\
\hline BLO-39-11-08-012 & 114 & 454 \\
\hline BLO-39-11-08-013 & 114 & 454 \\
\hline BLO-39-11-08-014 & $\overline{114}$ & 454 \\
\hline BLO-39-11-09-001 & 114 & 454 \\
\hline BLO-39-11-09-002 & $\overline{114}$ & 454 \\
\hline BLO-39-11-09-003 & 114 & 454 \\
\hline BLO-39-11-09-004 & 114 & 454 \\
\hline BLO-39-11-09-006 & 114 & 454 \\
\hline BLO-39-11-09-007 & 114 & 454 \\
\hline BLO-39-11-09-008 & 114 & 454 \\
\hline BLO-39-11-09-009 & 114 & 454 \\
\hline BLO-39-11-09-010 & $\overline{1114}$ & $4 \overline{454}$ \\
\hline BLO-39-11-09-011 & 114 & 454 \\
\hline BLO-39-11-09-012 & $\overline{114}$ & 454 \\
\hline BLO-39-11-09-013 & 114 & 454 \\
\hline
\end{tabular}




\begin{tabular}{|l|l|l|}
\hline $\begin{array}{l}\text { PFP THERMAL } \\
\text { STABILIZATION }\end{array}$ & BLEND PLAN & $\begin{array}{l}\text { HNF-4989, REV. 1 } \\
\text { PAGE 41 OF 51 }\end{array}$ \\
\hline
\end{tabular}

\begin{tabular}{|c|c|c|}
\hline \multicolumn{3}{|c|}{ VAULT 4} \\
\hline ITEMID & CAT & $\overline{\mathrm{COEI}}$ \\
\hline BLO-39-11-10-001 & 1114 & 454 \\
\hline BLO-39-11-10-002 & 1114 & $\overline{4454}$ \\
\hline BLO-39-11-10-003 & $\overline{114}$ & 454 \\
\hline BLO-39-11-10-004 & 114 & $\overline{454}$ \\
\hline BLO-39-11-10-005 & 114 & $\overline{454}$ \\
\hline BLO-39-11-10-006 & 114 & 454 \\
\hline BLO-39-11-10-007 & 114 & $\overline{454}$ \\
\hline BLO-39-11-10-008 & 114 & 4554 \\
\hline$\overline{\mathrm{BLO}-39-11-10-009}$ & $\overline{114}$ & $\overline{454}$ \\
\hline BLO-39-11-10-010 & 114 & $\overline{454}$ \\
\hline$\overline{\text { BLO-39-11-10-011 }}$ & 114 & $\overline{454}$ \\
\hline BLO-39-11-10-012 & 1114 & 454 \\
\hline$\overline{\text { BLO-39-11-11-001 }}$ & $\overline{114}$ & 454 \\
\hline BLO-39-11-11-002 & 114 & 454 \\
\hline BLO-39-11-11-003 & 114 & 454 \\
\hline$\overline{B L O}-39-11-11-004$ & 114 & $\overline{454}$ \\
\hline BLO-39-11-11-005 & 114 & 454 \\
\hline BLO-39-11-11-006 & $\overline{114}$ & 454 \\
\hline BLO-39-11-11-007 & 114 & 454 \\
\hline BLO-39-11-11-008 & 114 & $4 \overline{454}$ \\
\hline BLO-39-11-11-009 & 114 & 454 \\
\hline BLO-39-11-11-010 & $\overline{114}$ & 454 \\
\hline BLO-39-11-11-011 & $\overline{1114}$ & $\overline{454}$ \\
\hline BLO-39-11-11-012 & 1114 & $\overline{454}$ \\
\hline BLO-39-11-11-013 & $\overline{114}$ & 454 \\
\hline BLO-39-11-11-014 & 114 & 4554 \\
\hline BLO-39-11-12-001 & 114 & 454 \\
\hline BLO-39-11-12-002 & $\overline{\overline{114}}$ & 454 \\
\hline BLO-39-11-12-003 & $\overline{1114}$ & 454 \\
\hline BLO-39-11-12-004 & 1114 & $\overline{454}$ \\
\hline BLO-39-11-12-005 & $\overline{114}$ & 4454 \\
\hline
\end{tabular}




\begin{tabular}{|l|l|l|}
\hline $\begin{array}{l}\text { PFP THERMAL } \\
\text { STABILIZATION }\end{array}$ & BLEND PLAN & $\begin{array}{l}\text { HNF-4989, REV. 1 } \\
\text { PAGE 42 OF 51 }\end{array}$ \\
\hline
\end{tabular}

\begin{tabular}{|c|c|c|}
\hline \multicolumn{3}{|c|}{ VAULT 4} \\
\hline ITEMID & CAT & COEI \\
\hline BLO-39-11-12-006 & 114 & 454 \\
\hline BLO-39-11-12-007 & 114 & 454 \\
\hline BLO-39-11-12-008 & 114 & 454 \\
\hline BLO-39-11-12-009 & 114 & 4454 \\
\hline BLO-39-11-12-010 & 114 & 454 \\
\hline BLO-39-11-12-011 & 114 & 454 \\
\hline BLO-39-11-12-012 & 114 & 454 \\
\hline BLO-39-11-12-013 & 114 & 454 \\
\hline BLO-39-11-13-001 & $\overline{1114}$ & 454 \\
\hline BLO-39-11-13-002 & 114 & 454 \\
\hline BLO-39-11-13-003 & 114 & 454 \\
\hline BLO-39-11-13-004 & 114 & 454 \\
\hline 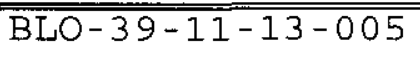 & 1114 & 454 \\
\hline BLO-39-11-13-006 & 114 & 454 \\
\hline BLO-39-11-13-007 & 114 & 4454 \\
\hline BLO-39-11-13-008 & 1114 & 454 \\
\hline BLO-39-11-13-009 & 114 & 454 \\
\hline BLO-39-11-13-010 & 114 & 454 \\
\hline BLO-39-11-13-011 & 1114 & 454 \\
\hline BLO-39-11-13-012 & 114 & 454 \\
\hline BLO-39-11-13-013 & 114 & 454 \\
\hline BLO-39-11-14-001 & 114 & 454 \\
\hline BLO-39-11-14-002 & 114 & 454 \\
\hline BLO-39-11-14-003 & 114 & 454 \\
\hline BLO-39-11-14-005 & 1114 & 454 \\
\hline BLO-39-11-14-006 & $\overline{114}$ & 454 \\
\hline BLO-39-11-14-007 & 114 & 454 \\
\hline BLO-39-11-14-008 & 114 & 454 \\
\hline BLO-39-11-14-009 & 114 & 454 \\
\hline BLO-39-11-14-010 & 114 & 4454 \\
\hline BLO-39-11-14-011 & 114 & 454 \\
\hline
\end{tabular}




\begin{tabular}{|l|l|l|}
\hline \hline $\begin{array}{l}\text { PFP THERMAL } \\
\text { STABILIZATION }\end{array}$ & BLEND PLAN & $\begin{array}{l}\text { HNF-4989, REV. 1 } \\
\text { PAGE 43 OF 51 }\end{array}$ \\
\hline
\end{tabular}

\begin{tabular}{|c|c|c|}
\hline \multicolumn{3}{|c|}{ VAULT 4} \\
\hline ITEMID & $\overline{\mathrm{CAT}}$ & COEI \\
\hline BLO-39-11-14-012 & $\overline{1114}$ & $\overline{2454}$ \\
\hline BLO-39-11-14-013 & 114 & 4554 \\
\hline BLO-39-11-14-014 & 114 & 454 \\
\hline BLO-39-11-15-001 & $\overline{114}$ & 454 \\
\hline BLO-39-11-15-002 & $\overline{1114}$ & 454 \\
\hline BLO-39-11-15-003 & $\overline{114}$ & $4 \overline{454}$ \\
\hline$\overline{B L O}-39-11-15-004$ & $\overline{114}$ & 454 \\
\hline$\overline{\mathrm{BLO}-39-11-16-001}$ & 114 & 454 \\
\hline$\overline{\mathrm{BLO}-39-11-16-002}$ & $\overline{114}$ & 454 \\
\hline BLO-39-11-16-003 & 114 & 454 \\
\hline BLO-39-11-16-007 & $\overline{114}$ & 454 \\
\hline$\overline{B L O}-39-11-16-008$ & 1114 & $\overline{454}$ \\
\hline$\overline{B L O}-39-11-16-010$ & 114 & 454 \\
\hline BLO-39-11-17-001 & $\overline{114}$ & 4454 \\
\hline BLO-39-11-17-002 & $\overline{114}$ & 454 \\
\hline BLO-39-11-17-003 & 1114 & 454 \\
\hline BLO-39-11-17-004 & 114 & 454 \\
\hline BLO $=39-11-17-005$ & 114 & $\overline{454}$ \\
\hline BLO-39-11-17-006 & 114 & 454 \\
\hline BLO-39-11-17-007 & 1114 & $\overline{454}$ \\
\hline BLO-39-11-17-008 & $\overline{1114}$ & $\overline{454}$ \\
\hline BLO-39-11-17-009 & 1114 & 454 \\
\hline BLO-39-11-17-010 & 114 & $\overline{454}$ \\
\hline BLO-39-11-17-011 & 114 & 454 \\
\hline BLO-39-11-17-012 & $\overline{114}$ & 454 \\
\hline BLO-39-11-17-013 & 114 & 454 \\
\hline$\overline{\mathrm{BLO}-39-11-17-014}$ & 114 & $\overline{454}$ \\
\hline BLO-39-11-17-015 & 114 & 454 \\
\hline $\mathrm{BO}-28-01-001-04$ & 150 & 454 \\
\hline BO-28-01-001-05 & 150 & 454 \\
\hline$\overline{\mathrm{BO}-28-01-001-06}$ & 150 & 454 \\
\hline
\end{tabular}


PFP THERMAL STABILIZATION
BLEND PLAN

HNF-4989, REV. 1

PAGE 44 OF 51

\begin{tabular}{|c|c|c|}
\hline \multicolumn{3}{|c|}{ VAULT 4} \\
\hline ITEMID & $\overline{\mathrm{CAT}}$ & COEI \\
\hline BO-28-01-001-07 & $\overline{150}$ & $\overline{454}$ \\
\hline BO-28-01-002-04 & 150 & 454 \\
\hline$\overline{\mathrm{BO}}-29-02-563-30$ & 150 & 4454 \\
\hline $\mathrm{BO}-29-03-058-\mathrm{B}$ & 150 & 454 \\
\hline $\mathrm{BO}-30-10-001-\mathrm{A}$ & 150 & 454 \\
\hline $\mathrm{BO}-30-10-001-\mathrm{B}$ & 150 & 454 \\
\hline $\mathrm{BO}-30-10-002-\mathrm{B}$ & 150 & 2454 \\
\hline $\mathrm{BO}-30-10-003-\mathrm{A}$ & 150 & 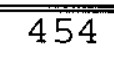 \\
\hline $\mathrm{BO}-30-10-003-\mathrm{B}$ & 150 & $\overline{4454}$ \\
\hline$\overline{\mathrm{BO}-30-10-004-\mathrm{B}}$ & 150 & 454 \\
\hline $\mathrm{BO}-30-10-005-1$ & 150 & 454 \\
\hline $\mathrm{BO}-30-10-005-2$ & 150 & 454 \\
\hline $\mathrm{BO}-30-10-006-1$ & 150 & 454 \\
\hline $\mathrm{BO}-30-10-006-2$ & 150 & 454 \\
\hline BO-30-10-007-1 & 150 & 4454 \\
\hline $\mathrm{BO}-30-10-007-2$ & 150 & 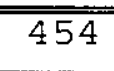 \\
\hline $\mathrm{BO}-30-10-008-1$ & 150 & 454 \\
\hline $\mathrm{BO}-30-10-008-2$ & 150 & 454 \\
\hline $\mathrm{BO}-30-11-012-1$ & 150 & 454 \\
\hline$\overline{\mathrm{BO}-30-11-012-2}$ & 150 & $\overline{454}$ \\
\hline BO-30-11-013-1 & 150 & 454 \\
\hline $\mathrm{BO}-30-11-013-2$ & 150 & 454 \\
\hline BO-30-11-014 & 150 & 454 \\
\hline $\mathrm{BO}-30-11-043-1=$ & 150 & 4454 \\
\hline$\overline{\mathrm{BO}-30-11-043-2}$ & 150 & 4454 \\
\hline$\overline{\mathrm{BO}-30-11-044-1}$ & 150 & 454 \\
\hline $\mathrm{BO}-30-11-044-2$ & 150 & 454 \\
\hline BO-30-11-045-1 & 150 & 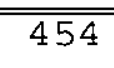 \\
\hline $\mathrm{BO}-30-11-045-2$ & 150 & 454 \\
\hline $\mathrm{BO}-30-11-046-1$ & 150 & 4454 \\
\hline $\mathrm{BO}-30-11-046-2$ & 150 & 454 \\
\hline
\end{tabular}




\begin{tabular}{|l|c|l|}
\hline \hline $\begin{array}{l}\text { PFP THERMAL } \\
\text { STABILIZATION }\end{array}$ & BLEND PLAN & $\begin{array}{l}\text { HNF-4989, REV. 1 } \\
\text { PAGE 45 OF 51 }\end{array}$ \\
\hline
\end{tabular}

\begin{tabular}{|c|c|c|}
\hline \multicolumn{3}{|c|}{ VAULT 4} \\
\hline ITEMID & CAT & $\overline{C \mathrm{COEI}}$ \\
\hline$\overline{B O}-30-11-047-1$ & 150 & 454 \\
\hline $\mathrm{BO}-30-11-047-2$ & 150 & 454 \\
\hline$\overline{B O}-30-11-047-3$ & 150 & 454 \\
\hline$\overline{\mathrm{BO}-30-11-048-1}$ & 150 & 454 \\
\hline $\mathrm{BO}-30-11-048-2$ & 150 & 454 \\
\hline$\overline{B O}-30-11-049$ & 150 & 454 \\
\hline $\mathrm{BO}-30-11-11-1$ & 150 & 454 \\
\hline BO-30-11-11-2 & 150 & 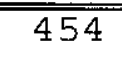 \\
\hline$B O-30-11-11-3$ & 150 & 454 \\
\hline DZO-35-09-002 & 175 & 454 \\
\hline$\overline{D Z O}-35-09-003$ & 1775 & $\overline{454}$ \\
\hline$\overline{\mathrm{DZO}-35-09-004}$ & 175 & 454 \\
\hline DZO-35-09-005 & 175 & $4 \overline{454}$ \\
\hline$\overline{D Z O-35-09-006}$ & 175 & 454 \\
\hline$\overline{D Z O-35-09-007}$ & 175 & 454 \\
\hline DZO-35-09-008 & 175 & $4 \overline{454}$ \\
\hline DZO-35-09-009 & $\overline{175}$ & 454 \\
\hline DZO-35-09-010 & 175 & $\overline{454}$ \\
\hline DZO-35-09-011 & 175 & 454 \\
\hline DZO-35-09-013 & 175 & 454 \\
\hline DZO-35-09-015 & 175 & 454 \\
\hline DZO-35-09-016 & 175 & 454 \\
\hline DZO-35-09-019 & 175 & 4554 \\
\hline DZO-35-09-020 & 175 & 454 \\
\hline DZO-35-09-021 & 175 & 454 \\
\hline DZO-35-09-022 & 175 & 454 \\
\hline DZO-35-09-023 & 175 & 454 \\
\hline$\overline{D Z O-35-09-024}$ & 175 & $4 \overline{454}$ \\
\hline D20-35-09-025 & 175 & 454 \\
\hline $020-35-09-026$ & 175 & 454 \\
\hline$\overline{\mathrm{DZO}-35-09-027}$ & 175 & 454 \\
\hline
\end{tabular}




\section{PFP THERMAL}

STABILIZATION

\section{BLEND PLAN \\ HNF-4989, REV. 1 \\ PAGE 46 OF 51}

\begin{tabular}{|c|c|c|}
\hline \multicolumn{3}{|c|}{$\overline{\text { VAULT }} 4$} \\
\hline ITEMID & $\overline{\mathrm{CAT}}$ & $\overline{\mathrm{COEI}}$ \\
\hline$\overline{D Z O-35-09-028}$ & $\overline{175}$ & 454 \\
\hline DZO-35-09-029 & 175 & 454 \\
\hline DZO-35-09-030 & 175 & 454 \\
\hline $\bar{D} 20-35-09-031$ & 175 & $\overline{4454}$ \\
\hline$\overline{D Z O}-35-09-032$ & 175 & 454 \\
\hline D20-35-09-033 & 1775 & 4454 \\
\hline$\overline{D Z O-35-09}-034$ & 175 & $\overline{454}$ \\
\hline$\overline{\mathrm{DZO}-35-09-035}$ & 175 & 454 \\
\hline$\overline{D Z 0-35-09-036}$ & 175 & $\overline{454}$ \\
\hline$\overline{D Z O-35-09-037}$ & 175 & $4 \overline{454}$ \\
\hline$\overline{D Z O-35-09-038}$ & 175 & $4 \overline{454}$ \\
\hline$\overline{D Z O-35-09-039}$ & 175 & 454 \\
\hline DZO-35-09-040 & 175 & 454 \\
\hline DZO-35-09-041 & 175 & 454 \\
\hline DZO-35-09-042 & 175 & 454 \\
\hline$\overline{D Z O-35-09-043}$ & 175 & 4454 \\
\hline $\mathrm{DZO}-35-09-044$ & 175 & 454 \\
\hline DZO-35-09-045 & 175 & 454 \\
\hline $\mathrm{DZO}-35-09-046$ & 175 & 454 \\
\hline$\overline{D Z Z O-35-09-047}$ & 175 & 454 \\
\hline $\mathrm{DZO}-35-09-048$ & 175 & 454 \\
\hline DZO-35-09-049 & 175 & 454 \\
\hline DZO-35-09-050 & 175 & 454 \\
\hline$\overline{\overline{D Z O}-35-09-051}$ & 175 & $4 \overline{454}$ \\
\hline DZO-35-09-052 & 175 & 454 \\
\hline DZO-35-09-053 & 175 & $4 \overline{454}$ \\
\hline DZO-35-09-054 & 175 & 454 \\
\hline DZO-35-09-055 & 175 & 4454 \\
\hline DZO-35-09-056 & 175 & 454 \\
\hline DZO-35-09-057 & 175 & 454 \\
\hline$\overline{D Z O-35-09-058}$ & $\overline{175}$ & $\overline{454}$ \\
\hline
\end{tabular}




\begin{tabular}{|l|l|l|}
\hline \begin{tabular}{l}
\hline PFP THERMAL \\
STABILIZATION
\end{tabular} & BLEND PLAN & $\begin{array}{l}\text { HNF-4989, REV. 1 } \\
\text { PAGE 47 OF 51 }\end{array}$ \\
\hline
\end{tabular}

\begin{tabular}{|c|c|c|}
\hline \multicolumn{3}{|c|}{ VAULT 4} \\
\hline ITEMID & $\mathrm{CAT}$ & COEI \\
\hline$\overline{\mathrm{DZO}-35-09-059}$ & 175 & 454 \\
\hline DZO-35-09-060 & 175 & 454 \\
\hline$\overline{D Z O-35-09-061}$ & 175 & 454 \\
\hline$\overline{\mathrm{DZO}-35-09-062}$ & 175 & 454 \\
\hline DZO-35-09-063 & 175 & 454 \\
\hline$\overline{D Z O}-35-09-064$ & 175 & 454 \\
\hline DZO-35-09-065 & 175 & 454 \\
\hline$\overline{D Z O-35-09-066}$ & 175 & 454 \\
\hline$\overline{D Z O}-35-09-067$ & 175 & 454 \\
\hline$\overline{D Z O}-35-09-068$ & 175 & 454 \\
\hline $\mathrm{DZO}-35-09-069$ & 175 & 454 \\
\hline$\overline{D Z O-35-09-070}$ & 175 & 454 \\
\hline$\overline{\mathrm{D} Z 0-35-09-071}$ & 175 & 454 \\
\hline$\overline{\mathrm{DZO}-35-09-072}$ & 175 & 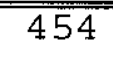 \\
\hline DZO-35-09-073 & 175 & 454 \\
\hline DZO-35-09-074 & 175 & "454 \\
\hline$\overline{\mathrm{DZO}-35-09-075}$ & 175 & 454 \\
\hline DZO-35-09-076 & 175 & 454 \\
\hline DZO-35-09-077 & 175 & 454 \\
\hline$\overline{D Z O-35-09-078}$ & 175 & 454 \\
\hline DZO-35-09-079 & 175 & 454 \\
\hline DZO-35-09-080 & 175 & 454 \\
\hline DZO-35-09-081 & 175 & 454 \\
\hline D20-35-09-082 & 175 & 454 \\
\hline DZO-35-09-287-A & 150 & 454 \\
\hline $\mathrm{DZO}-35-09-287-\mathrm{B}$ & 150 & 454 \\
\hline$\overline{D Z O}-35-09-294-\mathrm{A}$ & 150 & 454 \\
\hline DZO-35-09-294-B & 150 & 454 \\
\hline$\overline{\mathrm{DZO}-35-09-295-\mathrm{A}}$ & 150 & 454 \\
\hline $\mathrm{DZO}-35-09-295-\mathrm{B}$ & 150 & 454 \\
\hline DZO-35-09-301-A & 150 & 454 \\
\hline
\end{tabular}




\begin{tabular}{|l|l|l|}
\hline $\begin{array}{l}\text { PFP THERMAL } \\
\text { STABILIZATION }\end{array}$ & BLEND PLAN & $\begin{array}{l}\text { HNF-4989, REV. 1 } \\
\text { PAGE 48 OF 51 }\end{array}$ \\
\hline
\end{tabular}

\begin{tabular}{|c|c|c|}
\hline \multicolumn{3}{|c|}{ VAULT 4} \\
\hline ITEMID & CAT & COEI \\
\hline DZO-35-09-301-B & 150 & 454 \\
\hline $\mathrm{DZO}-35-09-302-\mathrm{A}$ & 150 & 454 \\
\hline$\overline{\mathrm{DZO}-35-09-302-\mathrm{B}}$ & 150 & 454 \\
\hline$\overline{D Z O}-35-09-303-\mathrm{A}$ & 150 & 454 \\
\hline DZO-35-09-303-B & 150 & 454 \\
\hline DZO-35-10-025-A & 175 & 454 \\
\hline $\mathrm{DZO}-35-10-026-\mathrm{A}$ & 175 & 454 \\
\hline$\overline{D Z O-35-10-027-A}$ & 175 & 454 \\
\hline$\overline{\mathrm{DZO}-35-10-028-\mathrm{A}}$ & 175 & 454 \\
\hline$\overline{D Z O}-35-10-053-\mathrm{A}$ & 175 & 454 \\
\hline$\overline{D Z O}-35-10-053-\mathrm{B}$ & 175 & 454 \\
\hline$\overline{D Z O}-35-10-054-\mathrm{B}$ & 175 & 2454 \\
\hline DZO-35-10-289-A & 150 & 454 \\
\hline DZO-35-10-289 B & 150 & $\overline{4454}$ \\
\hline DZO-35-10-291-A & 150 & $4 \overline{454}$ \\
\hline DZO-35-10-291-B & 150 & 454 \\
\hline $\mathrm{DZO}-35-10-296-\mathrm{A}$ & 150 & 454 \\
\hline$\overline{\mathrm{DZO}-35-10-297-\mathrm{A}}$ & 150 & 454 \\
\hline DZO-35-10-297-B & 150 & 454 \\
\hline $\mathrm{DZO}-35-10-298-\mathrm{A}$ & 150 & 454 \\
\hline $\mathrm{DZO}-35-10-298-\mathrm{B}$ & 150 & 4454 \\
\hline $\mathrm{DZO}-35-10-300-\mathrm{A}$ & 150 & 4454 \\
\hline $\mathrm{LAO}-\mathrm{CO} 2-251$ & 112 & 454 \\
\hline $\mathrm{LAO}-\mathrm{CO} 3-245$ & 112 & 454 \\
\hline $\mathrm{LAO}-\mathrm{CO} 4-253$ & 112 & 2454 \\
\hline $\mathrm{LAO}-\mathrm{CO} 5-246$ & 112 & 454 \\
\hline $\mathrm{LAO}-\mathrm{CO} 6-253$ & 1112 & 454 \\
\hline $\mathrm{LAO}-\mathrm{C0}-248$ & 112 & $\overline{2454}$ \\
\hline PBO-45-12-038-021 & 173 & 454 \\
\hline PBO-45-12-038-027 & 173 & $\overline{454}$ \\
\hline
\end{tabular}




\begin{tabular}{|l|l|l||}
\hline $\begin{array}{l}\text { PFP THERMAL } \\
\text { STABILIZATION }\end{array}$ & BLEND PLAN & $\begin{array}{l}\text { HNF-4989, REV. 1 } \\
\text { PAGE 49 OF 51 }\end{array}$ \\
\hline
\end{tabular}

\begin{tabular}{|c|c|c|}
\hline ITEMID & CAT & COEI \\
\hline $41-85-07-1106$ & 41 & $\mathrm{CO2}$ \\
\hline $41-87-06-250$ & 41 & $\mathrm{COI}$ \\
\hline $42-92-01-01-A$ & 42 & $\mathrm{COO}$ \\
\hline $42-92-01-01-B$ & 42 & $\mathrm{COO}$ \\
\hline $42-92-01-03$ & 42 & $\mathrm{COO}$ \\
\hline $42-94-11-89$ & 42 & $\mathrm{COO}$ \\
\hline $64-85-10-1596$ & 64 & $\mathrm{CO}$ \\
\hline $64-86-10-752$ & 64 & $\mathrm{COI}$ \\
\hline $64-96-01-017$ & 64 & $\mathrm{CO2}$ \\
\hline 839997 & 850 & 746 \\
\hline $88-83-06-319-1$ & 111 & 771 \\
\hline$B 02577 / B 02578$ & 74 & 746 \\
\hline $\mathrm{B} 02579 / \mathrm{B} 02580$ & 74 & 746 \\
\hline $\mathrm{B} 02581 / \mathrm{B} 02582$ & 73 & 746 \\
\hline $\mathrm{B} 02583 / \mathrm{B} 02584$ & 73 & 746 \\
\hline $\mathrm{B} 02585 / \mathrm{B} 02586$ & 73 & 746 \\
\hline $\mathrm{B} 02587 / \mathrm{B} 02588$ & 73 & 746 \\
\hline B3039 & 5 & 771 \\
\hline B3040 & 5 & 771 \\
\hline $\mathrm{DZO}-35-09-001$ & 175 & G01 \\
\hline $\mathrm{DZO}-35-09-001 \mathrm{~A}$ & 175 & G01 \\
\hline PPSL-394 & 51 & $\mathrm{CO} 2$ \\
\hline PPSL- 400 & $\underline{51}$ & $\mathrm{CO} 2$ \\
\hline $\mathrm{RA142}$ & 73 & GOO \\
\hline RA146-1B & 73 & $\mathrm{GOO}$ \\
\hline RA146C & 73 & G00 \\
\hline$Y E C-32-03-001-09$ & 175 & $\mathrm{GO1}$ \\
\hline
\end{tabular}

\begin{tabular}{|c|c|c|}
\hline \multicolumn{3}{|c|}{ Vault 225 } \\
\hline \hline ITEMID & CAT & COEI \\
\hline $1-X-1-6$ & 850 & 771 \\
\hline $2-X-1-6$ & 850 & 771 \\
\hline $3-X-1-6$ & 850 & 771 \\
\hline $4-X-1-6$ & 850 & 771 \\
\hline $5-X-1-6$ & 850 & 771 \\
\hline $17-X-5-6$ & 850 & 771 \\
\hline
\end{tabular}




\begin{tabular}{|l|l|l|}
\hline $\begin{array}{l}\text { PFP THERMAL } \\
\text { STABILIZATION }\end{array}$ & BLEND PLAN & $\begin{array}{l}\text { HNF-4989, REV. } 1 \\
\text { PAGE 50 OF 51 }\end{array}$ \\
\hline
\end{tabular}

\begin{tabular}{|c|c|c|}
\hline \multicolumn{3}{|c|}{ Vault 225} \\
\hline ITEMID & CAT & COEI \\
\hline $18-x-5-6$ & 850 & 771 \\
\hline $19-x-5-6$ & 850 & 771 \\
\hline $20-x-5-6$ & 850 & 771 \\
\hline $24-x-25-10$ & 850 & 771 \\
\hline $25-X-50-10$ & 850 & 771 \\
\hline $26-x-100-10$ & 850 & 771 \\
\hline $37-X A-25-6$ & 850 & 771 \\
\hline $38-X A-50-6$ & 850 & 771 \\
\hline $177-X A-9-8$ & 850 & 771 \\
\hline $178-X A-35-8$ & 850 & 771 \\
\hline $179-\mathrm{XA}-9-12$ & 850 & 771 \\
\hline $180-\mathrm{XA}-35-12$ & 850 & 771 \\
\hline $181-\mathrm{XA}-1-12$ & 850 & 771 \\
\hline $182-\overline{X A}-9-23$ & 850 & 771 \\
\hline $183-\mathrm{XA}-35-23$ & 850 & 771 \\
\hline $124-X S-11-8$ & 850 & 771 \\
\hline $125-\mathrm{XS}-20-8$ & 850 & 771 \\
\hline $126-X S-31-8$ & 850 & 771 \\
\hline $127-\mathrm{XS}-40-8$ & 850 & 771 \\
\hline $128-X S-51-8$ & 850 & 771 \\
\hline $129-X S-11-11$ & 850 & 771 \\
\hline $130-X S-20-11$ & 850 & 771 \\
\hline $132-X S-40-11$ & 850 & 771 \\
\hline $42-92-01-05$ & 42 & $\mathrm{COO}$ \\
\hline
\end{tabular}

\begin{tabular}{|c|c|c|}
\hline \multicolumn{3}{|c|}{ Vault 192C } \\
\hline \hline \multicolumn{1}{|c|}{ ITEMID } & CAT & COEI \\
\hline \hline $6-X-1-6$ & 850 & 771 \\
\hline $7-X-1-6$ & 850 & 771 \\
\hline $8-X-1-6$ & 850 & 771 \\
\hline $9-X-1-6$ & 850 & 771 \\
\hline $10-X-1-6$ & 850 & 771 \\
\hline $11-X-5-6$ & 850 & 771 \\
\hline $12-X-5-6$ & 850 & 771 \\
\hline $13-X-5-6$ & 850 & 771 \\
\hline
\end{tabular}




\begin{tabular}{|l|l|l|}
\hline $\begin{array}{l}\text { PFP THERMAL } \\
\text { STABILIZATION }\end{array}$ & BLEND PLAN & $\begin{array}{l}\text { HNF-4989, REV. 1 } \\
\text { PAGE 51 OF 51 }\end{array}$ \\
\hline
\end{tabular}

\begin{tabular}{|l|c|c|}
\hline \multicolumn{3}{|c|}{ Vault 192C } \\
\hline \hline ITEMID & CAT & COEI \\
\hline \hline $14-X-5-6$ & $\underline{850}$ & $\underline{7} 71$ \\
\hline $15-X-5-6$ & 850 & 771 \\
\hline $5-88-0,1-12$ & $\underline{5}$ & 605 \\
\hline
\end{tabular}




\section{DISTRIBUTION SHEET}

\begin{tabular}{l|l|l}
\hline To & From & Page 1 of 1 \\
\cline { 3 - 3 } Distribution & PFP Process Engineering & Date April 19, 2000 \\
\hline Project Title/Work Order & EDT No. N/A & ECN No. 657095 \\
\hline
\end{tabular}

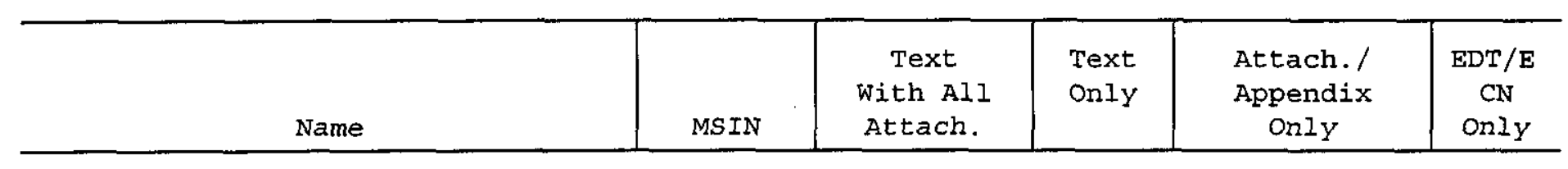

R. A. Burk

J. F. Durnil

G. E. Hickman Jr.

K. A. Larkin

N. E. Wilkins

A. L. Ramble

H. R. Risenmay

R. D. Redekopp

W. F. Russell Jr.

B. D. Skeels

D. R. Speer

A. M. Stubbs

R. W. Szempruch

M. D. Talbot

Central Files

\begin{tabular}{|c|c|}
\hline$T 4-20$ & $\mathrm{X}$ \\
\hline$T 5-55$ & $X$ \\
\hline T5-09 & $\mathrm{X}$ \\
\hline$T 5-55$ & $\mathrm{x}$ \\
\hline T5-55 & $\mathrm{x}$ \\
\hline T5-53 & $\mathrm{X}$ \\
\hline T5-55 & $\mathrm{X}$ \\
\hline T5-15 & $\mathrm{X}$ \\
\hline$T 5-51$ & $\mathrm{X}$ \\
\hline T5-09 & $\mathrm{X}$ \\
\hline$T 5-50$ & $\mathrm{x}$ \\
\hline $\mathrm{T} 5-55$ & $\mathrm{x}$ \\
\hline T5-55 & $\mathrm{x}$ \\
\hline T5-15 & $\mathrm{X}$ \\
\hline BI-07 & $\mathrm{X}$ \\
\hline
\end{tabular}

